\title{
Enquêtes d'acceptation de paiment destinées à déterminer les prix des produits et services dans le domaine de la santé de la reproduction : manuel de l'utilisateur
}

Karen G. Fleischman Foreit

James R. Foreit

Population Council

Follow this and additional works at: https://knowledgecommons.popcouncil.org/departments_sbsr-rh

Part of the Demography, Population, and Ecology Commons, and the International Public Health Commons

How does access to this work benefit you? Let us know!

\section{Recommended Citation}

Foreit, Karen G. Fleischman and James R. Foreit. 2005. "Enquêtes d'acceptation de paiment destinées à déterminer les prix des produits et services dans le domaine de la santé de la reproduction : manuel de I'utilisateur," FRONTIERS Final Report. Washington, DC: Population Council. 


\section{ENQUETES D'ACCEPTATION DE PAIMENT DESTINEES A DETERMINER LES PRIX DES PRODUITS ET SERVICES DANS LE DOMAINE DE LA SANTE DE LA REPRODUCTION}

\section{Manuel de I'utilisateur}

Karen G. Fleischman Foreit

POLICY Project

The Futures Group International
James R. Foreit

FRONTIERS

Population Council

Cette publication n'aurait pas été possible sans le support de l'AGENCE AMERICAINE POUR LE DEVELOPPEMENT INTERNATIONAL (USAID), sous les termes de l'Accord de coopération numéro HRN-A-00-98-0012-00, le programme Frontières de la santé de la reproduction du Population Council (FRONTIERS) et du contrat numéro HRN-C-00-00-00006-00, Projet POLICY de The Futures Group International.

Révisé 2005.

(C) 2005 par le Population Council. Tous droits réservés. 


\section{TABLE DES MATIERES}

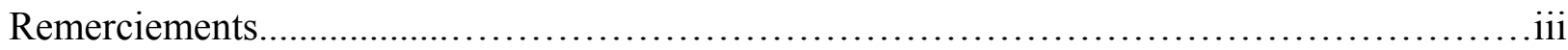

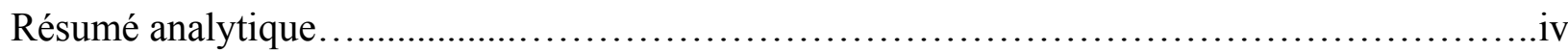

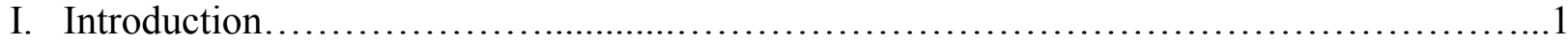

II. Enquêtes d'acceptation de paiement : De quoi s'agit-il ? ...................................

III. Enquêtes d'acceptation de paiement : Comment les mener.................................. 4

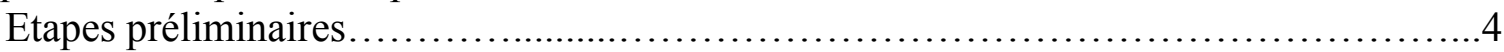

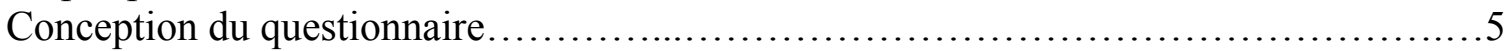

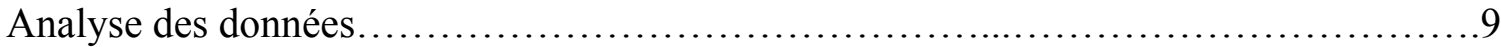

Estimation de la demande à différents niveaux de prix................................12

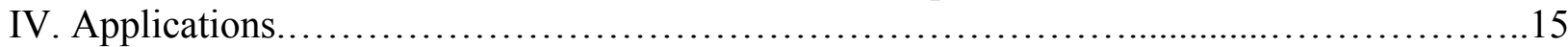

Mali : Augmentation des prix de produits existants ..............................16

Pakistan : Détermination de prix de produits nouveaux............................22

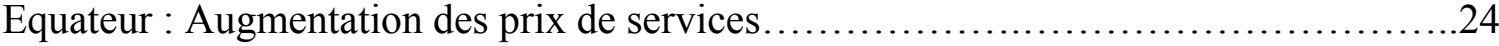

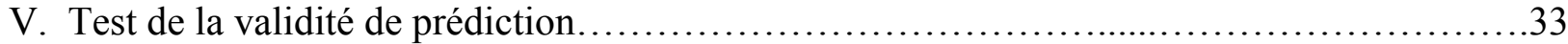

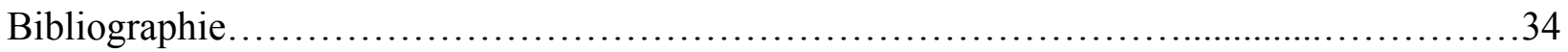

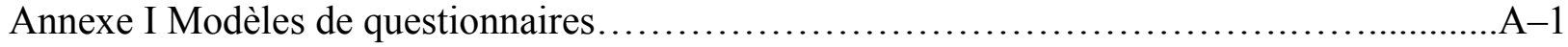

Annexe II Modèle de manuel de l'enquêteur/enquêtrice et modèle de manuel de codage......A-20 


\section{Remerciements}

Les auteurs désirent remercier nos collaborateurs pour le travail qu'ils ont effectué. Dan Kress, de Abt Associates, a attiré notre attention sur la technique d'acceptation de paiement (willingness to pay WTP) et a dirigé des enquêtes à ce sujet au Ghana et au Pakistan. John Bratt, de Family Health International, a joué un rôle de premier plan dans le cadre des enquêtes WTP qui ont été menées en Equateur et au Guatemala. Varuni Dayaratna, de The Futures Group International, Caroline Blair, du Population Reference Bureau, Carlos Brambila, du Population Council et Barbara Janowitz, de Family Health International, ont collaboré aux études WTP effectuées en Equateur, au Guatemala, au Mali et aux Philippines, respectivement. Nous désirons remercier tout particulièrement William Winfrey, lui aussi du The Futures Group International, pour les conseils qu'il a dispensés lors de plusieurs enquêtes et de la rédaction du présent document. Les enquêtes décrites dans le présent document ont été financées aux termes de divers contrats et de dons du Bureau Population, Santé et Nutrition de l'Agence des Etats-Unis pour le développement international (USAID), de l'USAID/Accra et de l'USAID/Bamako, sauf en ce qui concerne le Pakistan, qui a bénéficié d'un soutien accordé par un contrat DfID passé par le The Futures Group International. Le présent manuel a été élaboré dans le cadre du programme Frontières de la santé de la reproduction du Population Council (FRONTIERS) et du Projet POLICY de The Futures Group International, qui ont tous deux bénéficié d'un soutien accordé par l'USAID. 


\section{Résumé analytique}

Les programmes sociaux doivent arriver à un équilibre entre le volume (couverture) et les recettes (durabilité). D'après la loi de l'offre et de la demande, on ne peut pas avoir en même temps couverture et durabilité - lorsque les prix augmentent, la demande baisse. Il est inévitable que l'on perde des clients lorsque les prix augmentent, sauf dans les cas où ceux-ci sont si bas au départ ou que la demande est tellement élevée qu'elle n'est pas influencée par les changements de prix. Les enquêtes d'acceptation de paiement (willingness to pay - WTP) permettent aux directeurs de programmes de simuler les changements intervenant dans la demande en raison des prix sans les modifier réellement, ce qui leur donne un moyen de prendre des décisions relatives à la détermination des prix sur la base d'informations empiriques. Les enquêtes WTP présentent les avantages suivants :

- La méthodologie d'enquête est d'une mise en œuvre simple et discrète et les techniques d'estimation directe peuvent être appliquées par n'importe quelle unité de recherche.

- Les techniques WTP peuvent être appliquées aussi bien aux produits et services existants qu'à ceux qui sont nouveaux et non encore disponibles sur le marché. L'enquête peut être menée sur des échantillons basés sur la population ou les installations, en fonction du contexte du programme et des décisions à prendre.

- Même les clients n'ayant pas été scolarisés peuvent répondre à des questions hypothétiques sur les prix et leurs réponses sont habituellement convergentes.

- Les estimations WTP sont sensibles aux caractéristiques des clients, comme la motivation poussant à utiliser le produit ou le service ou le statut socioéconomique.

- Les estimations WTP directes sont prudentes en ce sens qu'elles sous-estiment le WTP maximum et protègent les directeurs de programmes contre une augmentation trop élevée des prix.

- Les informations provenant des enquêtes WTP rendent plus précise la prévision des réactions à un changement de prix. Les estimations ne permettent pas des prévisions parfaites, mais il semble que les erreurs soient systématiquement liées aux caractéristique du programme, comme le prix ou le volume initial.

Ceci dit, il faut cependant noter deux précautions à prendre :

1. La fourchette de prix hypothétique à tester devrait être déterminée en consultation avec la direction du programme avant de mener une enquête WTP exhaustive. Les prix ciblés devraient être basés sur des informations pertinentes concernant le programme, comme les coûts de la prestation du service ou de la remise du produit pour le programme, les besoins du programme en recettes supplémentaires destinées à couvrir l'augmentation des prix ou un financement transversal d'autres activités, les prix aux autres points de vente et ainsi de suite.

2. Il faut effectuer des études plus approfondies pour valider les résultats de toute décision prise en s'appuyant sur les enquêtes WTP. Les directeurs de programmes devraient, au minimum, suivre l'état de la demande avant, pendant et après les changements de prix. Il faut effectuer des expériences supplémentaires sur le terrain pour évaluer à quel point les estimations de changement utilisées, qui sont basées sur les enquêtes WTP sont précises et sensibles. 


\section{Introduction}

Lorsqu'ils prennent des décisions en matière d'établissement de prix, les directeurs de programmes sociaux sont confrontés à un dilemme d'équité - arriver à un équilibre entre la nécessité d'avoir un programme durable et l'objectif social qui consiste à mettre des services à la disposition de clients à faible revenu. Une augmentation trop importante des prix interdira aux clients pauvres l'accès aux services. D'un autre côté, maintenir des prix inutilement bas impose un recours perpétuel aux bailleurs de fonds externes. Jusqu'à récemment, les directeurs de programmes étaient obligés de prendre des décisions en matière d'établissement de prix sans pouvoir se reposer sur une méthode fiable de prévision des effets des changements de prix sur les recettes ou l'utilisation du programme. Le présent document décrit une méthode d'enquête simple, qui donne une estimation de l'acceptation de paiement (WTP) par les clients pour les biens et services, ce qui leur permet de prendre des décisions rationnelles dans ce domaine.

Fixer les prix est une décision majeure pour tout programme fournissant des biens et des services. Alors que les administrateurs d'entreprises commerciales font de leur mieux pour maximiser les profits, augmenter leur part du marché ou décourager la concurrence, ceux d'organisations publiques et à but non lucratif se concentrent sur la capacité de payer, la durabilité et la couverture assurée. Le défi auquel les programmes sociaux sont confrontés consiste à fixer des prix suffisamment bas pour que la clientèle ciblée ait les capacités de payer, mais qui soient cependant suffisamment élevés pour éviter d'avoir à cannibaliser les marques et services financièrement autonomes et entièrement commerciaux. La fourchette potentielle des prix peut être très étendue et il n'y a habituellement pas de prix optimal qu'un programme puisse demander.

La « loi de l'offre et de la demande » stipule que la demande est inversement proportionnelle au prix. Lorsque les prix augmentent, les ventes ou l'utilisation baissent et, inversement, quand ils baissent, les ventes augmentent. Ce rapport peut cependant ne pas être applicable aux extrêmes. Il est possible qu'il y ait un prix minimum en dessous duquel d'autres réductions n'entraînent pas un volume plus important de ventes. ${ }^{1}$ De même, lorsque les prix sont tellement élevés que plus personne n'achète rien, d'autres augmentations ne risquent pas de réduire les ventes. Nous nous attendons donc à ce que le rapport entre les prix et les ventes ressemble à la « courbe de la demande » présentée à la Figure $1 .{ }^{2}$

\footnotetext{
${ }^{1}$ Oliver (1994) a constaté que la réduction à zéro du prix des spermicides dans les installations du ministère de la Santé du Ghana n'a augmenté que le 5 pourcent la probabilité d'utilisation d'un moyen de contraception moderne, probablement parce que les prix étaient très bas pour commencer. D'autres chercheurs ont fait état de résultats similaires en Thaïlande (Akin et Schwartz 1988), en Indonésie (Jensen et al. 1993) et en Equateur (Bratt et al. 1998 ; Leon et Cuesta 1993).

${ }^{2}$ Les économistes placent traditionnellement le prix sur l'axe vertical (Y). Dans le présent document, nous placerons les ventes sur l'axe Y, ce qui reflète la tradition des spécialistes du comportement, qui placent la variable dépendante sur cet axe. Dans la loi de l'offre et de la demande, les ventes sont la variable dépendante parce que la variable est considérée comme étant déterminée ou influencée par les prix.
} 


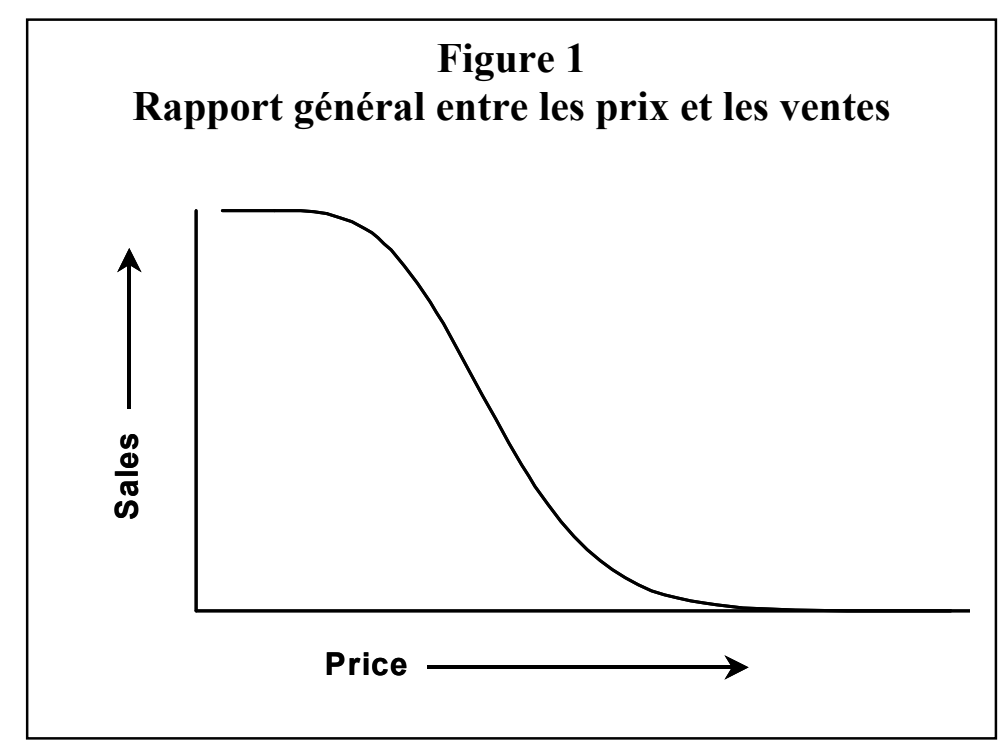

Sales - Ventes

Price - Prix

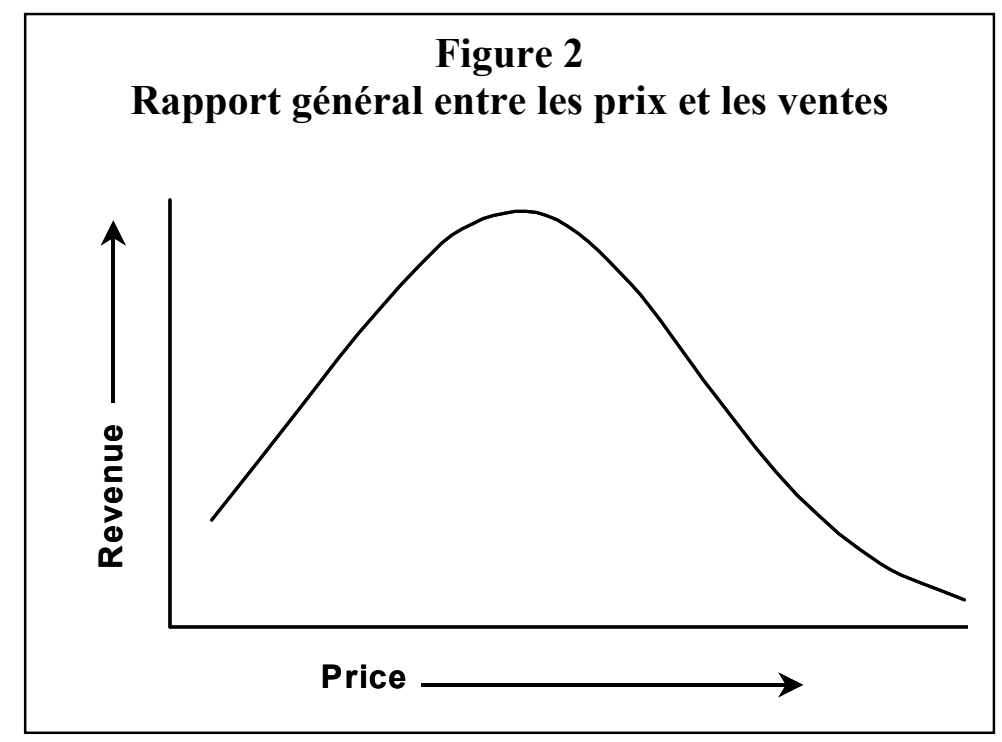

La forme de la courbe de la demande pour un produit ou un service donné dépendra de nombreux facteurs, dont notamment les autres méthodes, marques et sources

d'approvisionnement, aussi bien que le revenu des clients et le niveau de motivation de l'utilisation du produit ou du service. S'il existe de nombreux choix concurrents sur le marché et que le revenu du client est faible, il se peut que la vente ou l'utilisation chute en cas d'augmentation des prix. D'un autre côté, si les choix sont restreints et que les clients sont motivés, il se peut que les ventes ou l'utilisation soient moins affectées par un changement de prix.

Les recettes attendues sont calculées en multipliant le nombre attendu de ventes par le prix unitaire de ce qui est vendu. En fonction de la forme de la courbe de la demande, dans une certaine fourchette de prix, la hausse du prix compensera largement la baisse des ventes et cette augmentation entraînera des recettes plus importantes. Pour d'autres gammes de prix, de petites hausses des prix entraîneront une baisse relativement importante des ventes et des prix plus élevés se solderont par une baisse des recettes.

Revenue - Recettes

Price - Prix

Le fait de connaître la forme et la hauteur de la courbe des recettes permet aux directeurs de programmes d'estimer les coûts qu'ils recouvreront à différents niveaux de prix. (Figure 2). ${ }^{3}$ Un grand nombre de programmes ont des objectifs de durabilité aussi bien que d'accès. Pouvoir prévoir la réaction des clients aux niveaux de prix aiderait leurs directeurs à estimer l'impact qu'une hausse de prix proposée pourrait avoir sur les recettes aussi bien que sur l'utilisation du produit ou du service.

\footnotetext{
${ }^{3}$ Les recettes ne constituent qu'un seul élément de l'équation déterminant la durabilité. Il se peut que les coûts du programme n'indiquent pas une correspondance biunivoque avec les ventes et il est donc possible que les bénéfices (ou les pertes) nets ne reflètent pas exactement les recettes.
} 
Comment pouvons-nous savoir ce que sera le comportement des consommateurs en matière d'achat en fonction de différents prix ? Empiriquement, nous pourrions augmenter les prix et mesurer le niveau des ventes et continuer à augmenter les prix jusqu'à ce que la demande chute à un niveau inacceptable ou que les recettes commencent à baisser, et revenir ensuite à un prix moins élevé. De toute évidence, la plupart des directeurs de programmes hésiteraient à jouer de cette façon avec leur clientèle et leurs recettes. Il existe un autre moyen d'aborder le problème : demander aux clients actuels ou potentiels ce qu'ils seraient prêts à payer pour le produit ou le service recherché. ${ }^{4}$

\section{Enquêtes d'acceptation de paiement : De quoi s'agit-il ?}

Les enquêtes WTP mesurent la demande potentielle en produits ou services en demandant aux consommateurs « achèteriez-vous ce produit s'il était offert à tel prix ? Elles sont fréquemment utilisées par les programmes de santé, sociaux et environnementaux pour la détermination des prix et les analyses de rentabilité. Lorsqu'il s'agit d'effectuer quelques analyses simples, les données d'enquêtes WTP permettent aux directeurs de programmes d'estimer le nombre de clients qui seront prêts à payer un prix donné, le montant des recettes provenant de ce prix et, si le questionnaire comprend les questions appropriées, les caractéristiques des personnes qui sont prêtes - et de celles qui ne le sont pas - à payer ce prix. Dans le cadre d'analyses plus complexes, les données peuvent aussi être utilisées pour estimer le prix d'un produit ou d'un service qui maximisera les recettes. L'enquête WTP de base s'effectue rapidement et n'exige que des échantillonnages relativement modestes.

\section{Enquêtes d'acceptation de paiement : Comment les mener}

\section{Etapes préliminaires}

Les enquêtes d'acceptation de paiement répondent à la question "Quels prix pouvons-nous demander? » Avant d'essayer d'y répondre grâce à une enquête WTP, il nous faut la réponse à une autre question : "Quel prix devrions-nous demander? » Les prix à tester dans le cadre d'une telle enquête devraient être basés sur un objectif ou un autre du programme. Il faut que cet objectif soit clairement défini avant de concevoir l'enquête d'acceptation de paiement.

Il faut en outre poser deux questions essentielles au directeur du programme : "Qui désirons-nous servir?» et «Qu'avez-vous l'intention de faire avec les recettes produites par le prix demandé ou sa hausse ? » Elles permettent de focaliser l'enquête sur la population ciblée par le programme et sur ses objectifs financiers.

Connaître la population ciblée. La plupart des prestataires de service essayent d'atteindre un type particulier de clients. Les programmes sociaux - ministères de la Santé et organisations non gouvernementales (ONG — définissent souvent leur marché cible en termes de revenu, de capacité à payer ou de manque d'accès à des points de vente commerciaux. Etant donné que différents groupes de clients peuvent réagir différemment aux augmentations de prix, le fait de spécifier à l'avance quelle est la population ciblée permet à l'enquêteur d'inclure ces caractéristiques dans l'enquête et de concentrer l'analyse sur les personnes les plus importantes pour le programme.

\footnotetext{
${ }^{4}$ Cette façon d'aborder la WTP est aussi appelée « préférence exprimée ».
} 
Connaître vos objectifs financiers. Le plus souvent, les prix sont augmentés pour rester au niveau de la croissance des coûts ou de l'inflation, pour accroître le recouvrement des coûts afin de moins dépendre des bailleurs de fonds externes (durabilité) ou de générer des revenus supplémentaires afin d'étendre les services ou de subventionner d'autres activités du programme. Tout ceci exige que les directeurs de programmes sachent quels sont leurs coûts réels, ce qui est en soi une étude en discipline. Si le but du nouveau prix est d'acheter des contraceptifs, il faut alors que le directeur sache combien ils coûtent. S'il s'agit de générer un excédent de recettes pour subventionner un autre service, il faut alors savoir quel est le prix de chaque service. Finalement, si le but est d'améliorer la durabilité du programme, il faut alors connaître tous ses coûts, y compris les produits et fournitures utilisés lors des consultations ou fournis au client (dont les prix de gros payés ou la valeur des donations, de l'emballage, du transport, du stockage, etc.) et les coûts secondaires associés à la prestation des services (salaires, loyer et services publics, équipement, maintenance, etc.). Il existe plusieurs moyens de classer les coûts du programme, comme par exemple les coûts fixes (comme les salaires et le loyer, qui demeurent les mêmes, quel que soit le nombre de clients vus ou les services fournis) et les coûts variables (associés au service fourni, comme le prix des produits et fournitures utilisés).

Sélectionner un petit nombre de produits ou de services. Les programmes de marketing ne s'intéressent le plus souvent à l'acceptation de paiement que pour un seul ou un nombre limité de produits. Par contre, les programmes cliniques cherchent parfois à fixer ou à augmenter les prix d'un grand nombre de produits et services. Il est possible d'en inclure beaucoup dans une enquête WTP, mais il vaut normalement mieux se concentrer sur trois ou quatre services prioritaires au maximum. Les services dont le volume est faible et contribuant peu aux coûts ou aux recettes ne devraient pas en faire partie parce qu'il est difficile dans de tels cas d'avoir un échantillonnage suffisamment important pour permettre une prise de décision fiable.

Connaître vos prix actuels. Les prix imposés dans les points de vente d'un programme peuvent ne pas être les mêmes que ceux qui ont été fixés au siège de l'organisation. Les données relatives aux prix en vigueur au niveau central sont souvent dépassées ou incomplètes. Il faut appeler chaque dispensaire devant faire partie de l'étude ou s'y rendre, pour obtenir les véritables prix courants de tous les services couverts par l'enquête. Il arrive souvent, dans les cas de marketing social ou commercial, qu'un produit soit offert à des prix différents en fonction de leur point de vente. Il faut, avant de commencer l'enquête, obtenir des informations fiables sur la gamme de prix réels du produit visé.

Connaître le volume actuel et passé du service. Il est indispensable de disposer de statistiques précises ou de registres des ventes si l'on veut interpréter correctement les données de l'enquête. La demande pour de nombreux produits de santé, comme les sels de réhydratation orale ou les moustiquaires de lits, peut fluctuer en fonction des saisons et les heures d'ouverture des dispensaires peuvent être réduites lors des jours fériés pour célébrations civiles ou religieuses. Les augmentations de prix peuvent avoir des effets différents dans les nouveaux emplacements où la demande est plus importante qu'au niveau des sites établis où elle a été constante ou même en baisse.

Sélectionner les stratégies d'échantillonnage. Le type et l'importance de l'échantillonnage utilisé dans une enquête WTP dépendront de la nature du produit ou du service qui en fait l'objet et de la précision des résultats requis. La conception de l'étude doit être déterminé par la programmation. Si le directeur d'un dispensaire veut connaître l'impact d'une augmentation des prix sur sa clientèle actuelle, l'endroit approprié pour mener l'enquête est le dispensaire lui-même. Une enquête relative à la détermination des prix de produits appelés à être vendus dans les pharmacies peut être menée auprès des clients qui quittent ces établissements (ce que l'on appelle une enquête d'interception). On peut mieux étudier l'introduction de nouveaux produits ou services parmi les personnes qui ne les utilisent actuellement pas, grâce à des échantillons représentatifs de la population du pays dans son ensemble ou de zones géographiques données ou ayant un statut socioéconomique particulier. 
En règle générale, plus les échantillons sont importants, plus les estimations sont précises et les coûts de l'enquête augmentent avec le nombre de produits ou de services et de points de prestation de service qui y sont inclus. Les enquêtes menées dans les dispensaires ont tendance, pour des raisons d'efficacité, à poser des questions aux clients qui en sortent après avoir obtenu un service dans un certain nombre d'établissements pendant une période donnée (habituellement entre 2 et 4 semaines, en fonction du volume de la clientèle). Il s'agit là d'un choix viable si le dispensaire a une importante clientèle, mais il se peut qu'il ne soit pas rentable d'obtenir un nombre fiable de réponses dans les petits points de vente tels que les distributeurs communautaires ou les cliniques rurales qui peuvent ne pas desservir plus d'un ou deux clients par jour.

Sélectionner une enquête ou un module autonome dans le cadre d'une enquête plus étendue. On peut faire des économies en incluant le jeu de base de questions WTP dans une enquête menée à d'autres fins, comme par exemple une étude du niveau de satisfaction des clients à la sortie d'un dispensaire de soins de santé de la reproduction. Les possibilités de collaboration sont cependant souvent limitées par les caractéristiques des échantillonnages et le moment auquel l'enquête plus importante est menée.

\section{Conception du questionnaire}

La National Oceanic and Atmospheric Administration (Administration nationale des Etats-Unis des océans et de l'atmosphère - NOAA) a établi les règles des meilleures pratiques visant à surmonter les grandes sources de partialité associées aux enquêtes WTP. ${ }^{5}$ Elle recommande que les enquêtes WTP 1) se reposent sur des entretiens personnels, 2) fassent appel à des questions fermées amenant la personne interrogée à réagir à une augmentation donnée pour un service qu'elle connaît, 3) rappellent aux personnes interrogées que l'augmentation de prix réduit d'autres consommations, 4) rappellent aux personnes interrogées que le service en question peut être remplacé par autre chose, et 5) posent aux personnes interrogées des questions sur les facteurs qui pourraient avoir une influence sur leur préférence.

La plupart des organismes de recherche requièrent que la personne interrogée donne son consentement en toute connaissance de cause avant que l'entretien puisse avoir lieu. Un tel consentement est un processus de communication entre le chercheur et le participant potentiel et est aussi une prescription juridique aux Etats-Unis. La personne prenant part à l'étude doit être informée du but de celle-ci, comprendre l'information et c'est à elle de prendre une décision volontaire quant à sa participation ou non à l'enquête. Le consentement en toute connaissance de cause trouve son origine dans la recherche médicale, qui comprend des risques et des avantages. Les principaux éléments d'un consentement en toute connaissance de cause sont les suivants :

1. But de l'étude.

2. Risques encourus par le participant à l'étude, y compris les risques physiques, sociaux et émotionnels.

3. Avantages que le participant peut tirer de l'étude, y compris, si nécessaire, une déclaration selon laquelle il n'en existe pas.

4. Informations relatives à la confidentialité, y compris l'utilisation d'identificateurs et l'accès à des données personnelles.

5. Où aller à tout moment en cas de question ou de problème.

\footnotetext{
${ }^{5}$ A la suite de la marée noire provoquée par l'Exxon Valdez, le Département du Commerce des Etats-Unis a réuni un groupe d'experts afin de déterminer si les pertes estimées par la méthode WTP étaient suffisamment fiables dans le cadre d'une évaluation portant sur les dommages causés à une ressource naturelle. Voir Portnoy (1994) et Phillips et al. (1997). Les directives décrites dans le présent document cherchent à minimiser la partialité des réponses si les personnes interrogées ne comprennent pas les questions, si elles ne peuvent pas y répondre, si elles n'y répondent pas honnêtement ou si elles répondent de façon «stratégique » afin que les résultats de l'étude soient en leur faveur.
} 
6. Informations concernant le retrait de l'étude. Le participant a le droit de décider de ne plus en faire partie à quelque moment que ce soit, sans pénalisation.

7. Garantie que les services sont disponibles, que le participant potentiel décide ou non de prendre part à l'étude ou s'il décide de ne plus y participer.

8. Informations sur les procédures suivies et la durée de l'étude.

Les formulaires de consentement doivent utiliser des phrases simples et des déclarations directes, appropriées au niveau d'alphabétisation ou de scolarisation des personnes interrogées. Il faut donner au participant l'occasion de poser des questions et il doit être bien informé avant de signer le formulaire de consentement. Un exemple de formulaire de consentement en toute connaissance de cause figure en Annexe I.

Dans la pratique, il faut que les personnes interrogées comprennent le contexte dans lequel la question est posée, c'est-à-dire qu'elles doivent comprendre exactement ce qu'elles achèteraient. Ceci est moins problématique lorsque l'on demande à des utilisateurs actuels s'ils sont prêts à continuer à acheter un produit ou un service à un prix plus élevé ou si l'on pose la question à des personnes qui s'en servaient dans le passé, mais ne le font plus. Il peut cependant être difficile de poser des questions sur quelque chose que les personnes interrogées n'ont jamais utilisé, comme dans le cas d'un produit nouveau. Dans le cas d'enquêtes WTP relatives à des produits nouveaux, il vaut mieux en montrer un échantillon à la personne interrogée et lui en faire une description complète. Il faut aussi commencer, lorsque l'on se trouve en présence d'une personne qui n'utilise pas le produit, par déterminer si elle s'y intéresse ou non.

La façon dont les questions relatives aux prix sont posées peut aussi avoir une influence sur les réponses données. Poser aux personnes interrogées une seule question, directe et ouverte — « Quel est le maximum que vous êtes prêt à payer pour ce produit? »-ne donne pas de réponses fiables. Les chercheurs sont d'accord sur le fait qu'il vaut mieux poser des questions sur des prix donnés et demander aux personnes interrogées de répondre par oui ou par non (ou je ne sais pas). L'enquête devrait comporter suffisamment de questions pour couvrir les fourchettes de prix envisagées, sans toutefois fatiguer inutilement la personne interrogée. Il faut aussi choisir l'ordre dans lequel les questions sont posées, de façon à ne pas forcer la personne interrogée à donner un prix maximum plus élevé ou plus bas qu'elle ne le pense réellement.

L'entretien devrait commencer en demandant à la personne interrogée quel est le montant qu'elle paie actuellement pour le produit ou le service. Il faudrait ensuite lui demander si elle serait prête à continuer à acheter le produit ou le service si son prix était augmenté d'un montant donné. Il faudrait ensuite demander aux personnes interrogées si elles achèteraient toujours le produit ou le service si son prix était augmenté d'un montant déterminé. On demande ensuite à celles qui répondent par l'affirmative si elles payeraient un montant encore plus élevé. On demande alors aux personnes qui disent non ou ne sont pas sures, si elles seraient prêtes à payer moins, mais un prix toujours plus élevé que le prix courant. Il faut noter qu'exactement trois questions relatives aux prix sont posées à toutes les personnes interrogées : celui qu'elles paient actuellement $[\mathrm{P}(0)]$, une augmentation moyenne $[\mathrm{P}(2)]$ et, en fonction de la réponse à l'augmentation moyenne de prix, une hausse faible $[\mathrm{P}(1)]$ ou élevée $[\mathrm{P}(3)]$. Aucune personne n'est interrogée sur les quatre prix et ceux qui sont mentionnés varieront en fonction des divers prix payés actuellement par les personnes interrogées.

La Figure 3 illustre l'ordre dans lequel les questions sont posées aux personnes interrogées qui utilisent actuellement un produit ou un service. Dans le cas de celles qui ne l'utilisent pas pour le moment, il faudrait leur demander si elles s'y intéressent ; les questions WTP commencent avec la question numéro 2 de la Figure 3 et les prix mentionnés sont absolus au lieu d'être des augmentations. 


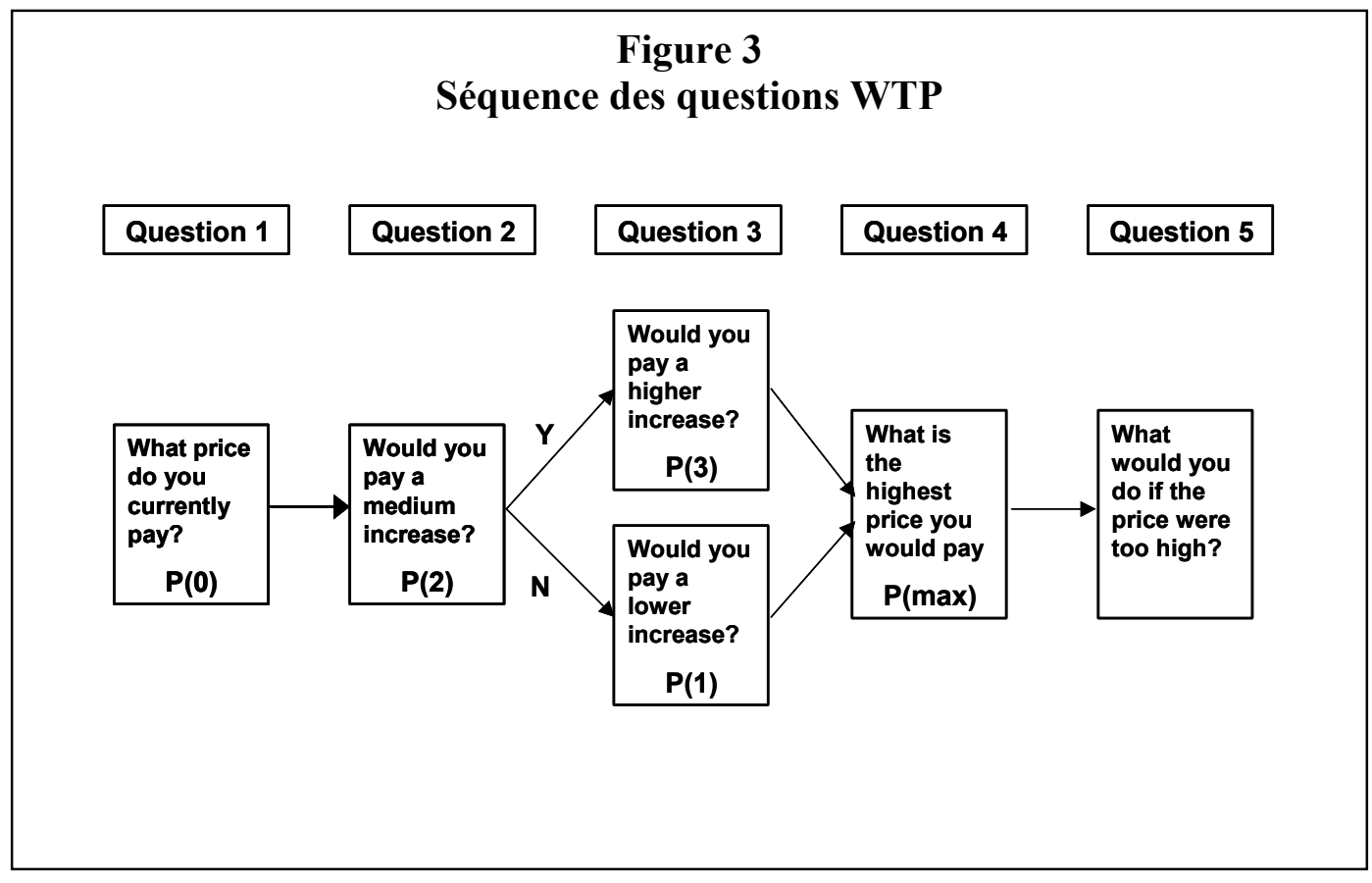

$\mathrm{P}(0)$ - Quel prix payez-vous actuellement?

$\mathrm{P}(2)$ - Accepteriez-vous de payer une légère augmentation?

$\mathrm{P}(3)$ - Accepteriez-vous de payer une augmentation plus importante?

$\mathrm{P}(1)$ - Accepteriez-vous de payer une augmentation moins importante ?

$\mathrm{P}(\max )$ - Quel est le prix maximum que vous accepteriez de payer?

Que feriez-vous si le prix était trop élevé ?

Lors de l'élaboration du questionnaire, le chercheur devrait mentionner le prix ciblé dans la question relative à une augmentation de prix faible ou dans celle de l'augmentation moyenne, mais pas dans celle qui a trait à la hausse la plus élevée. L'expérience suggère que les usagers et ceux qui peuvent l'être dans les pays en développement ont souvent tendance à traiter les questions WTP comme s'il s'agissait d'un marchandage. Ils sont davantage prêts à payer un prix donné si l'augmentation est faible plutôt que moyenne (premiers prix) ou la plus élevée.

Il est fortement recommandé de poser deux autres questions portant sur les prix. La première consiste à demander à toutes les personnes interrogées, quelle que soit leur réponse à la question numéro 3 , quel est le prix maximum qu'elles seraient prêtes à payer. Ceci étend la gamme des prix pour celles qui répondent oui au prix le plus fort. Pour celles qui répondent non au dernier prix mentionné, ceci permet de faire une distinction plus fine entre le prix accepté le plus élevé et le plus faible qui ne l'est pas. Les données portant sur le prix maximum peuvent aussi servir à vérifier la convergence des réponses (dans l'idéal, le nombre de personnes interrogées mentionnant un prix maximum inférieur à celui qu'elles paient actuellement, ou l'augmentation acceptée pour les questions fermées devrait être infime). Finalement, on peut demander aux personnes interrogées ce qu'elles feraient si les prix étaient augmentés à un niveau supérieur à celui qu'elles sont prêtes à payer. Cette question peut aider un programme privé à établir sa niche sur le marché ou un directeur de programme public cherchant à estimer à quel point un filet de sécurité sociale s'avère nécessaire. 
Il faut toujours inclure dans les enquêtes WTP des questions portant sur les caractéristiques des clients. Elles permettent de découvrir celles des personnes interrogées qui sont prêtes ou non à payer un prix donné, et servent souvent à déterminer leur richesse, leur revenu discrétionnaire ou leur capacité à payer. Les indicateurs les plus couramment utilisés en ce qui concerne la richesse comprennent le niveau d'éducation, le revenu familial, la possession de biens durables, les dépenses récentes pour d'autres services et produits relatif à la santé et les dépenses pour des choses qui ne sont pas essentielles, comme les salons de beauté. Les questions spécifiques qui doivent faire partie de l'enquête dépendent du pays où elle est menée. Les enquêtes démographiques et sanitaires (EDS) sont une bonne source de questions relatives à l'éducation et aux biens durables. Des exemples sont aussi fournis dans le questionnaire modèle pour les services de clinique (voir Annexe I). Il existe une relation étroite entre l'éducation, le revenu et la possession de biens durables et le chercheur peut donc vouloir n'utiliser qu'une seule question (comme celle qui porte sur la variable relative à l'éducation) lorsque l'entretien doit être effectué dans un temps très limité ou que les questions relatives au revenu pourraient être très indiscrètes.

Il faut ajouter au questionnaire WTP des questions supplémentaires permettant d'explorer, lors de l'entretien, l'intérêt ou la motivation des usagers potentiels d'un produit ou d'un service. Il est peu probable que les informations collectées auprès de personnes n'ayant pas l'intention d'acheter le produit ou le service soient d'une quelconque utilité. On peut demander aux personnes interrogées de classer leur intérêt (par exemple, « Diriez-vous que vous êtes très intéressé, quelque peu intéressé ou pas du tout intéressé par une stérilisation dans les deux prochaines années ? ») ou de répondre par oui ou par non à une question leur demandant si elles s'intéressent à l'utilisation d'un moyen de contraception ou d'un autre service de santé de la reproduction. Le chercheur voudra probablement exclure les personnes peu motivées de l'analyse d'acceptation de paiement.

La technique WTP mentionnée ci-dessus part de l'hypothèse que seul le prix varie et que tous les autres facteurs restent inchangés. Les chercheurs et directeurs peuvent aussi vouloir savoir si les clients seraient prêts à payer davantage en cas d'amélioration de certains aspects du service, comme réduire le temps d'attente ou installer des sièges plus confortables, et quels sont les facteurs entraînant une meilleure acceptation de paiement. Dans ce cas, la technique de recherche préférée est l'analyse conjointe, dans laquelle on demande à la personne interrogée de choisir entre deux combinaisons de prix et de caractéristiques de service, comme par exemple « Est-ce que vous préférez payer 5 dollars pour votre visite pour une pose de stérilet, être assise sur des chaises en plastique dur et attendre une heure, ou payer 15 dollars et être assise dans une chaise rembourrée et n'attendre que 10 minutes ? » Le nombre de questions posées dépend de celui des prix et caractéristiques du service qui en fait l'objet, mais l'évaluation de deux prix et deux caractéristiques requiert un minimum de huit questions.

\section{Analyse des données}

\section{Méthode d'estimation directe pour le calcul de la courbe de la demande}

L'analyse des clients actuels et potentiels s'effectue de la même façon. Pour calculer la courbe de la demande, nous partons des hypothèses suivantes :

- Les clients sont prêts à payer le prix qu'ils règlent actuellement pour leur produit ou leur service.

- Le prix maximum pour une personne est le plus élevé qu'elle a explicitement agréé. Si une personne interrogée répond non pour 4 dollars et oui pour deux, le prix maximum est de 2 dollars.

- L'acceptation de paiement est transitif vers le bas : les clients qui sont prêts à payer un prix donné 
pour un produit ou un service le sont aussi à tout prix inférieur pour ce même produit ou service.

- Le refus de payer est transitif vers le haut : les clients qui ne sont pas prêts à payer un prix donné pour un produit ou un service ne le sont pas non plus à tout prix plus élevé.

Une fois ces hypothèses de départ prises en compte, il faut suivre les étapes ci-après :

1. Etablir le prix maximum que la personne interrogée est prête à payer. ${ }^{6}$ Si la question porte sur des augmentations de prix, le prix maximum que les personnes interrogées sont prêtes à payer est calculé en ajoutant l'augmentation maximum au prix payé actuellement. Si la question porte sur des prix absolus, le prix maximum que les personnes interrogées sont prêtes à payer est le maximum qui a été donné.

2. Eliminer les non réponses et celles qui ne sont pas convergentes (toujours oui). Il faut supprimer de l'analyse les personnes interrogées répondant à toutes les questions par je ne sais pas. Par contre, il faut inclure les personnes interrogées répondant je ne sais pas aux questions normalisées sur les prix, mais qui indiquent le maximum qu'elles sont prêtes à payer, et il en va de même pour celles qui répondent par oui ou non aux questions relatives aux prix, mais ne peuvent pas indiquer quel est le montant maximum qu'elles sont prêtes à payer. Dans ce dernier cas, il faudrait estimer que le prix maximum est celui qui est le plus élevé mentionné dans les questions et accepté ou le prix actuel si aucun prix de l'enquête n'est accepté.

Il faut vérifier la convergence de toutes les réponses donnant un prix WTP maximum. Le prix maximum déclaré doit être égal ou supérieur au prix le plus élevé accepté lors de l'enquête. Les personnes interrogées indiquant un prix maximum inférieur à ce dernier ou au prix courant doivent être éliminées de l'analyse.

Il faut noter le pourcentage de personnes interrogées ayant été éliminées pour n'avoir pas donné de réponses ou pour avoir donné des réponses non convergentes. S'il est tellement important que les conclusions en sont affectées de façon substantielle, l'enquête elle-même est d'une utilité douteuse et il faut chercher les raisons pour lesquelles les personnes interrogées ont éprouvé des difficultés avec le questionnaire.

3. Effectuer une distribution de fréquence du prix WTP maximum.

4. Déterminer les tranches de prix qui figureront sur l'axe $x$ de la courbe de la demande, comme indiqué à la Figure 1. Pointer le pourcentage de la demande à chaque augmentation de prix en utilisant le complément de la fréquence cumulative de la distribution de fréquence et des règles relatives à la décision de transitivité mentionnées précédemment. Le pourcentage de personnes acceptant le prix $x$ est calculé (100 moins le pourcentage de celles dont le prix maximum est inférieur à $x$ ). Ce calcul est illustré au Tableau 1.

\footnotetext{
${ }^{6}$ La présente section part de l'hypothèse que tous les entretiens prennent fin sur une question ouverte: «Quel est le prix [augmentation] maximum que vous êtes prêt à payer?» (Question 4, Figure 3). L'analyse devient plus complexe si la question ouverte n'est pas posée à toutes les personnes interrogées et impose la suppression de certaines personnes interrogées de l'analyse aux niveaux de prix plus élevés.
} 


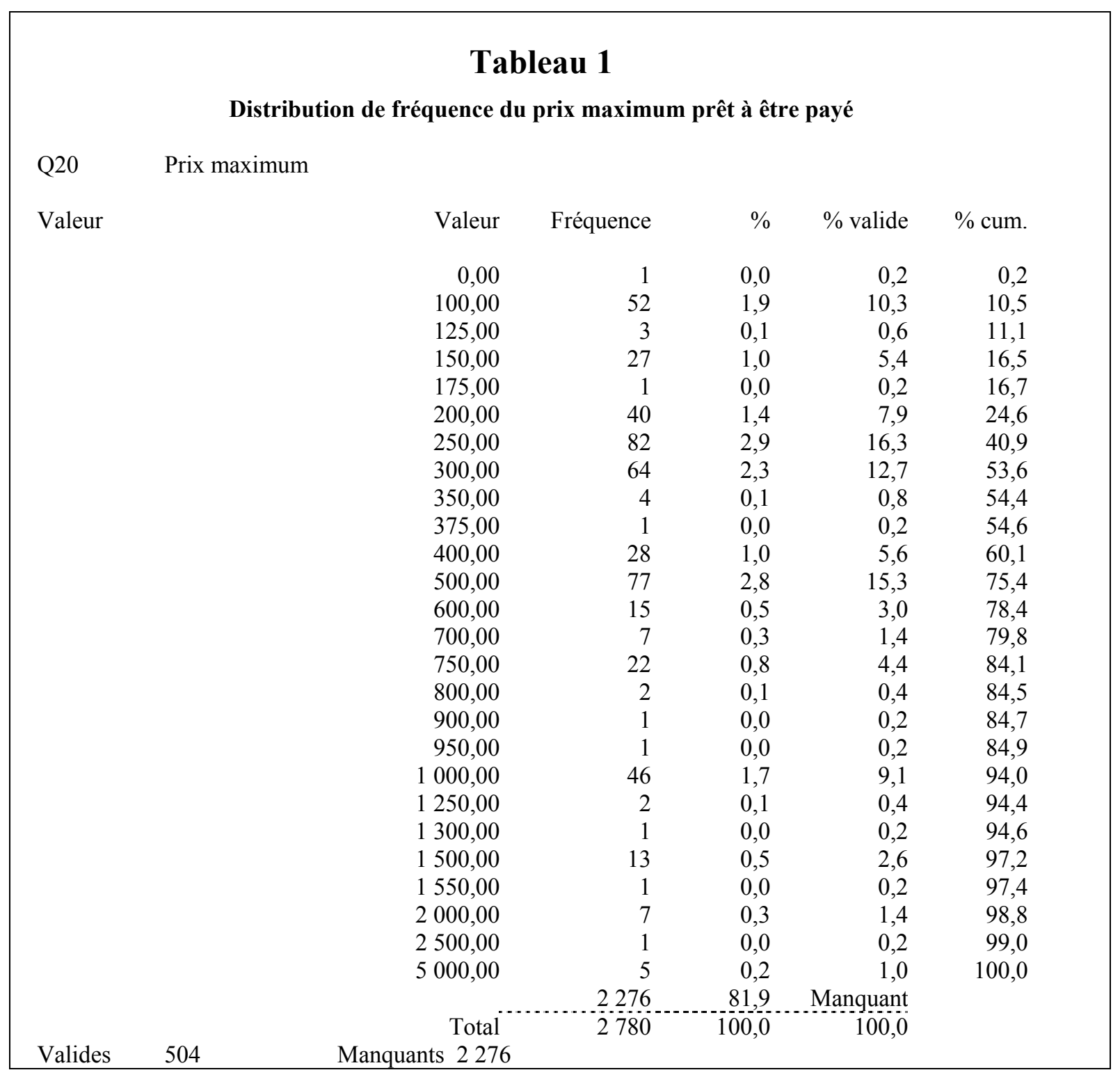

Supposons que nous voulons tracer la courbe de la demande par tranches de 50 francs d'augmentation des prix, à partir de 50 francs. Une personne interrogée (pourcentage cumulatif de 0,2 ) donne un prix maximum de 0 , ce qui fait que le pourcentage de personnes prêtes à payer 50 francs est de $100-0,2=$ 99,8. Comme personne n'a donné de prix maximum situé entre 0 et 100 francs, le pourcentage de personnes prêtes à payer 100 francs est de 99,8. Le prix maximum déclaré par 52 personnes interrogées a été de 100 francs, et de 125 pour trois autres, ce qui fait que le pourcentage des personnes prêtes à payer 150 francs est de $100-11,1=88,9$. Le pourcentage des personnes prêtes à payer 200 francs est le complément du pourcentage cumulatif donnant un prix inférieur (175 francs) : $100-16,7=83,3$ et ainsi de suite.

Les personnes interrogées peuvent de la même façon être réparties en fonction de leur statut socioéconomique et on peut comparer les courbes de la demande qui en résultent afin de déterminer l'impact de différents prix sur les clients ou obtenir des profils de clients potentiels. 
La méthode d'analyse directe décrite ci-dessus aura tendance à sous-estimer la WTP maximum parce que peu de questions sont posées sur les prix proposés et de nombreuses personnes interrogées peuvent simplement redonner le prix accepté le plus élevé proposé comme étant le maximum qu'elles sont prêtes à payer. Les économistes du domaine sanitaire qui effectuent des analyses de rentabilité ont souvent besoin d'une estimation plus fine de la WTP maximum. ${ }^{7}$ C'est la raison pour laquelle ils ont tendance à utiliser des techniques s'appuyant sur le modèle de régressions multiples au lieu de l'estimation directe. La plupart des programmes sociaux ne s'intéressent cependant ni à la maximisation des recettes ni aux analyses de rentabilité. Si le directeur de programme a déjà déterminé le prix ciblé et l'a inclus dans l'enquête en tant que prix le plus bas, ces méthodes donneront des estimations prudentes des prix WTP dans la fourchette visée.

Un programme aura souvent des prix différents pour le même service en fonction du lieu où il est offert. Selon les besoins du directeur, les personnes interrogées peuvent être regroupées pour tous les sites où le service est offert afin de calculer une seule courbe de la demande (en calculant d'abord le prix maximum prêt à être payé en ajoutant au prix actuel l'augmentation la plus élevée qui a été acceptée ou mentionnée) ou des courbes séparées pour chaque site ou groupe de sites ayant les mêmes prix.

\section{Estimation de la demande à différents niveaux de prix}

L'analyse WTP mentionnée ci-dessus établit une demande relative à différents prix (c'est-à-dire le pourcentage de personnes interrogées prêtes à payer chaque prix pour le bien ou le service). L'estimation de la demande absolue - combien de clients le programme aura-t-il à un prix donné - requiert une source supplémentaire et indépendante de données. Pour les ventes ou la clientèle courantes, il s'agit du volume actuel (pondéré pour tenir compte des variations saisonnières ou des tendances récentes).

Suivant la loi « de l'offre et de la demande », nous partons de l'hypothèse que le volume des clients baissera en cas d'augmentation des prix. Nous pouvons obtenir une estimation du volume des nouveaux clients à des prix plus élevés en combinant la courbe globale de la demande et les statistiques relatives au service, comme indiqué ci-après :

1. Estimer la demande globale pour le prix courant et celui qui est proposé. Si toutes les personnes interrogées payent actuellement le même prix, la demande au prix courant est de 100 pourcent. Par contre, si des prix différents sont demandés en fonction des sites, la demande globale au prix courant dans une installation où les prix sont élevés sera inférieure à 100 pourcent.

2. Calculer la proportion de la demande globale au nouveau prix divisée par la demande globale au prix courant. Le complément de la proportion de la demande ainsi obtenu (100 - proportion) est le pourcentage de déclin que l'on peut prévoir si les prix sont augmentés à ce nouveau niveau.

3. Multiplier la proportion de la demande par le volume actuel de service. Le produit de cette opération est le volume prévu de service auquel on peut s'attendre si les prix sont augmentés au nouveau niveau.

4. Multiplier le prix actuel par le volume actuel du service. Si tous les clients payent ce prix, on a les recettes actuellement générées. Multiplier le volume prévu pour le service par le nouveau prix proposé. Si tous les clients payent le nouveau prix, on a les recettes prévues générées au nouveau niveau de prix.

\footnotetext{
${ }^{7}$ La WTP est souvent utilisée en économie sanitaire pour donner une valeur monétaire à un avantage que le client tire d'un bien ou d'un service. Cette valeur monétaire peut être définie comme étant le prix maximum que le client serait prêt à payer.
} 
Si l'on peut prévoir combien ou quel pourcentage de clients nous perdrions en cas d'augmentation des prix, l'inverse n'est pas nécessairement vrai — on ne peut pas prévoir, à partir d'un échantillonnage pris au niveau de l'installation, combien de clients nous gagnerions en cas de baisse des prix actuels. Pour mobiliser la demande supplémentaire potentielle, il faut estimer combien de personnes n'utilisent actuellement pas le service pour la seule raison de son prix courant et quel montant elles seraient prêtes à payer pour y avoir recours. Une enquête basée sur la population pourrait nous fournir cette information, mais il faut noter que les enquêtes telles que l'EDS constate constamment que le prix est une raison mineure pour la non utilisation d'un moyen de contraception.

Il faudrait, pour les nouveaux produits ou services, mesurer notre marché potentiel de façon indépendante. Le refus d'achat d'un nouveau produit à son prix fixé inclura aussi bien les personnes qui ne sont pas prêtes à payer le prix en question que celles qui ne sont pas prêtes à l'utiliser à quelque prix que ce soit, même s'il était fourni gratuitement. La mesure supplétive de l'importance du marché pourrait être la proportion de personnes interrogées qui semblent très intéressées par l'utilisation du produit ou du service ; la demande relative à divers niveaux de prix pourrait être comparée pour ce sous-groupe, comme précédemment, et extrapolée à l'ensemble de la population.

\section{Suivi de la progression de l'enquête}

Tous les projets doivent être planifiés et faire l'objet d'un suivi et les enquêtes WTP ne font pas exception. La liste de vérification donnée ci-après peut aider le chercheur à planifier et à assurer le suivi de l'enquête. La première section donne la liste des problèmes que les décideurs faisant partie du programme doivent résoudre avant que l'on puisse concevoir l'enquête. Chaque question figurant dans la première colonne exige une réponse dans la deuxième. Par exemple, la première de la colonne Politique demande pourquoi il faut appliquer ou augmenter les prix. Si la réponse est « Recouvrement des coûts », la question suivante leur demande d'expliquer quels sont les coûts à recouvrer.

La seconde section, Conception de l'enquête, donne les éléments qui doivent être sélectionnés avant le début de l'enquête, comme « quels sont les dispensaires qui devront être inclus ? » Des installations particulières figureront sous Décision relative à la conception de l'enquête. La troisième section, Exécution de l'enquête, donne la liste des tậches à accomplir pendant celle-ci, y compris les entretiens avec les clients et la mise au propre et la saisie des données. Les personnes ou organisations responsables de ces tậches figurent dans la deuxième colonne. La dernière est réservée pour les dates de fin des travaux. 
Tableau 2

Enquête WTP : Liste de contrôle des décisions et tậches

\begin{tabular}{|c|c|c|}
\hline I. Politique & Décision (de référence) & Date de fin des travaux \\
\hline $\begin{array}{l}\text { Pourquoi demander un } \\
\text { prix pour les services? }\end{array}$ & $\begin{array}{l}\text { Recouvrement des coûts } \\
\text { Accroissement des recettes } \\
\text { Autre }\end{array}$ & \\
\hline $\begin{array}{l}\text { Si recouvrement des coûts, } \\
\text { lesquels? }\end{array}$ & $\begin{array}{l}\text { Variables? } \\
\text { Articles? }\end{array}$ & \\
\hline \multicolumn{3}{|l|}{ Quels services? } \\
\hline \multicolumn{3}{|l|}{ Dans quelles installations? } \\
\hline $\begin{array}{l}\text { II. Conception de } \\
\text { l'enquête }\end{array}$ & Décision de conception & \\
\hline \multicolumn{3}{|l|}{ Couverture géographique } \\
\hline \multicolumn{3}{|l|}{$\begin{array}{l}\text { Couverture installation } \\
\text { (types) }\end{array}$} \\
\hline \multicolumn{3}{|l|}{$\begin{array}{l}\text { Unité d'analyse ou de } \\
\text { ventilation }\end{array}$} \\
\hline \multicolumn{3}{|l|}{$\begin{array}{l}\text { Importance de } \\
\text { l'échantillon par cellule }\end{array}$} \\
\hline \multicolumn{3}{|l|}{ Flux client quotidien } \\
\hline \multicolumn{3}{|l|}{ Budget } \\
\hline $\begin{array}{l}\text { III. Exécution de } \\
\text { l'enquête }\end{array}$ & Responsabilité d'exécution & \\
\hline \multicolumn{3}{|l|}{$\begin{array}{l}\text { Conception du } \\
\text { questionnaire }\end{array}$} \\
\hline \multicolumn{3}{|l|}{$\begin{array}{l}\text { Détermination de } \\
\text { l'échantillonnage }\end{array}$} \\
\hline \multicolumn{3}{|l|}{ Travail sur le terrain } \\
\hline \multicolumn{3}{|l|}{$\begin{array}{l}\text { Saisie ou mise au propre } \\
\text { des données }\end{array}$} \\
\hline \multicolumn{3}{|l|}{ Analyse des données } \\
\hline \multicolumn{3}{|l|}{ Préparation du rapport } \\
\hline Dissémination & & \\
\hline
\end{tabular}




\section{Applications}

La technique WTP a été très utilisée ces dernières années par le groupe de recherche sanitaire de Family Health International, The Futures Group International et le programme FRONTIERS de the Population Council afin d'aider les directeurs de programmes des pays en développement à prendre des décisions relatives à l'établissement des prix des produits et services dans le domaine de la santé de la reproduction. Parmi les autres groupes qui appliquent des méthodes similaires, on peut citer International Planned Parenthood Federation (IPPF), région hémisphère occidental et le Commercial Market Strategies Project (CMS). Les informations relatives à ces enquêtes et à leur application figurent au Tableau 3. Le reste de cette section présente trois études de cas relatives à cette méthode, qui comprennent des conclusions sur la fiabilité et la validité de cette technique.

\section{Tableau 3}

\section{Récentes enquêtes WTP dans le domaine de la santé de la reproduction}

\begin{tabular}{|c|c|c|c|}
\hline Pays/Agence/Année & Echantillonnage & $\begin{array}{l}\text { Produits/ } \\
\text { Services }\end{array}$ & $\mathrm{AT}^{*}$ \\
\hline $\begin{array}{l}\text { Ghana: GSMF 1995, Marketing } \\
\text { social de produits nouveaux et } \\
\text { existants destinés à la contraception }\end{array}$ & $\begin{array}{l}\text { Echantillon national } \\
\text { représentatif de } 3016 \\
\text { femmes }\end{array}$ & $\begin{array}{l}\text { Fourniture de } \\
\text { contraceptifs } \\
\text { Préservatifs féminins }\end{array}$ & TFGI \\
\hline $\begin{array}{l}\text { Pakistan : Projet PPSPP, 1996, } \\
\text { Marketing social de produits } \\
\text { nouveaux destinés à la } \\
\text { contraception }\end{array}$ & $\begin{array}{l}\text { Echantillon représentatif de } \\
1753 \text { femmes mariées } \\
\text { vivant en milieu urbain et } \\
\text { périurbain }\end{array}$ & $\begin{array}{l}\text { Fourniture de } \\
\text { contraceptifs }\end{array}$ & TFGI \\
\hline $\begin{array}{l}\text { Equateur : CEMOPLAF, 1997, } \\
\text { Services de clinique ONG }\end{array}$ & $\begin{array}{l}\text { Echantillon systématique } \\
\text { de } 3661 \text { femmes dans } 15 \\
\text { cliniques }\end{array}$ & $\begin{array}{l}\text { Stérilet, prénatal, } \\
\text { gynécologie, WTP pour } \\
\text { attentes moins longues et } \\
\text { conseils supplémentaires }\end{array}$ & $\begin{array}{l}\text { FHI } \\
\text { PC } \\
\text { TFGI }\end{array}$ \\
\hline $\begin{array}{l}\text { Guatemala : APROFAM, 1999, } \\
\text { Services de clinique ONG }\end{array}$ & $\begin{array}{l}\text { Echantillons systématiques } \\
\text { en clinique ; la première } \\
\text { enquête a porté sur } 4856 \\
\text { femmes dans } 18 \text { cliniques } \\
\text { la seconde sur } 1827 \\
\text { femmes dans six cliniques }\end{array}$ & $\begin{array}{l}\text { Prénatal, gynécologie, } \\
\text { frottis vaginaux, } \\
\text { fourniture de } \\
\text { contraceptifs, Norplant }\end{array}$ & $\begin{array}{l}\text { FHI } \\
\text { PC }\end{array}$ \\
\hline $\begin{array}{l}\text { Mali : Pilplan, 1999, Projet de } \\
\text { marketing social de contraceptifs }\end{array}$ & $\begin{array}{l}\text { Echantillon représentatif de } \\
2780 \text { femmes et } 2780 \\
\text { hommes }\end{array}$ & $\begin{array}{l}\text { Fourniture de } \\
\text { contraceptifs }\end{array}$ & TFGI \\
\hline $\begin{array}{l}\text { Guatemala : Ministère de la Santé, } \\
\text { 2000, centres de santé hospitaliers } \\
\text { sans lit et polycliniques }\end{array}$ & $\begin{array}{l}\text { Echantillon systématique } \\
\text { de } 2468 \text { femmes dans six } \\
\text { cliniques }\end{array}$ & $\begin{array}{l}\text { Stérilisation féminine, } \\
\text { contraceptifs } \\
\text { temporaires, ultrasons }\end{array}$ & $\begin{array}{l}\text { FHI } \\
\text { PC }\end{array}$ \\
\hline $\begin{array}{l}\text { Philippines : Cliniques de sages- } \\
\text { femmes du programme familles en } \\
\text { bonne santé, } 2000\end{array}$ & $\begin{array}{l}\text { Echantillon systématique } \\
\text { de } 2277 \text { femmes dans } 60 \\
\text { cliniques }\end{array}$ & $\begin{array}{l}\text { Diagnostics de la } \\
\text { grossesse, prénatal, } \\
\text { accouchement, frottis } \\
\text { vaginaux, contraceptifs } \\
\text { temporaires }\end{array}$ & $\begin{array}{l}\text { FHI } \\
\text { PC }\end{array}$ \\
\hline
\end{tabular}

*AT (assistance technique) $:$ FHI $=$ Family Health International, programme FRONTIERS ; PC $=$ Population Council, programme INOPAL III ou FRONTIERS ; TFGI = The Futures Group International. 


\section{Mali : Augmentation des prix de produits existants}

Le projet de marketing social financé par l'USAID au Mali a mis sur le marché une marque de préservatifs (Protector) en 1992 et un contraceptif par voie orale (Pilplan) en 1993. Au début du projet, les prix ont été fixés à 100 francs maliens (FM) par cycle de Pilplan et à 50 FM pour un paquet de trois préservatifs Protector, soit approximativement 0,17 et 0,09 dollar américain, respectivement à la mi-1998.

La conception du projet prévoyait un passage en revue de la fixation des prix, devant avoir lieu à la fin de 1998, afin d'améliorer la durabilité du projet et la distribution. Une enquête WTP a été effectuée dans trois sites urbains et les zones rurales environnantes, notamment Sikasso, Mopti et la capitale, Bamako. Sikasso et Mopti ont été sélectionnées parce qu'elles représentaient respectivement les zones ayant les ventes les plus fortes et les plus faibles. L'échantillon comprenait 2780 femmes ậgées de 15 à 40 ans et 2750 hommes ậgés de 15 à 45 ans. Il a été stratifié par zone urbaine et zone urbaine-rurale; des secteurs de dénombrement ont été choisis au hasard dans les strates.

La séquence des questions posées aux utilisateurs actuels de pilules, de produits injectables et de préservatifs a suivi l'ordre donné à la Figure 3, page 12. Il a été demandé aux femmes n'utilisant pas actuellement la pilule ou de produits injectables si elles envisageaient de faire appel à l'une ou l'autre de ces méthodes à l'avenir ; on a aussi demandé aux hommes n'utilisant pas de préservatifs actuellement s'ils envisageaient de le faire à l'avenir. Un prix maximum a été calculé pour chaque personne interrogée utilisant actuellement ou pouvant être un utilisateur potentiel de chaque méthode.

\section{Contraceptifs par voie orale}

Sur 2780 femmes sexuellement actives ayant été interrogées, 390, soit 14 pourcent, ont déclaré utiliser actuellement des contraceptifs par voie orale. Pratiquement toutes celles qui prenaient la pilule ont pu donner des détails sur leur achat le plus récent - cinq seulement (1,3\%) n'ont pas pu donner la marque de la pilule qu'elles prenaient et cinq autres n'ont pas pu donner le prix payé le plus récemment. La marque vendue dans le cadre du plan de marketing social, Pilplan, arrivait nettement en tête du marché avec 71 des utilisatrices actuelles. La plupart des utilisatrices de cette marque $(65 \%)$ ont déclaré que la source la plus récente de leur approvisionnement était une pharmacie et 98 pourcent d'entre elles ont déclaré avoir payé $100 \mathrm{FM}$ pour un cycle de pilules, soit le prix de détail établi.

Toutes les utilisatrices actuelles de la pilule ont pu donner une réponse à la première augmentation de prix hypothétique -79 pourcent ont déclaré qu'elles accepteraient une augmentation de $100 \mathrm{FM}$ et 21 pourcent qu'elles ne l'accepteraient pas. Après avoir répondu à deux questions directes relatives au prix, il leur a été demandé à toutes de donner le prix le plus élevé qu'elles seraient prêtes à payer. Celui-ci a été comparé à l'augmentation la plus forte que la personne interrogée accepterait. Aux fins de convergence, il faut que le prix maximum soit égal ou supérieur à l'augmentation de prix acceptée la plus élevée (plus le prix original) ; 93 pourcent des personnes interrogées ont donné une réponse convergente ( $45 \% \mathrm{~s}$ 'en sont tenues au dernier prix mentionné et $48 \%$ en ont donné un plus élevé).

Le taux d'alphabétisation est faible chez les femmes du Mali : 60 pourcent seulement de celles utilisant actuellement la pilule ont déclaré avoir été scolarisées. Quoi qu'il en soit, la scolarisation n'a pas été associée à l'aptitude à répondre au questionnaire. La probabilité que les femmes n'ayant pas fréquenté l'école connaissaient le prix de leur contraceptif par voie orale n'a pas été plus faible que pour celles qui avaient été scolarisées ( $1 \%$ par rapport à $2 \%$ ) et elle n'a été que légèrement supérieure en ce qui concernait les réponses qui ne sont pas convergentes aux questions WTP $(8 \%$ contre $4 \%, p<0,07)$. 
Quelques rares utilisatrices actuelles de la pilule ont déclaré que leur dernière source d'approvisionnement n'était pas celle à laquelle elles faisaient appel habituellement (7 personnes interrogées, soit $2 \%$ ). Elles ont été supprimées de l'analyse ultérieure car il s'agissait d'utilisatrices ne pouvant pas donner le dernier prix payé ou dont les réponses n'étaient pas convergentes. L'acceptation de paiement a été analysée pour les 92 pourcent d'utilisatrices actuelles de la pilule qui répondaient à tous les critères d'éligibilité (comme par exemple, source d'approvisionnement constante, aptitude à donner le dernier prix payé et réponse convergente à propos des prix). Elles ont été classées en trois catégories socioéconomiques sur les biens durables qu'elles possédaient et leur niveau d'éducation. ${ }^{8}$ La Figure 4 compare la WTP entre les utilisatrices actuelles de contraceptifs par voie orale vivant en zone urbaine, par couche socioéconomique. Les différences ne sont pas importantes, mais il faut cependant noter que la probabilité d'acceptation d'augmentations hypothétiques de prix est la plus élevée pour les femmes de la couche socioéconomique la plus élevée que pour celles que la couche inférieure, et pour toute la gamme des prix.

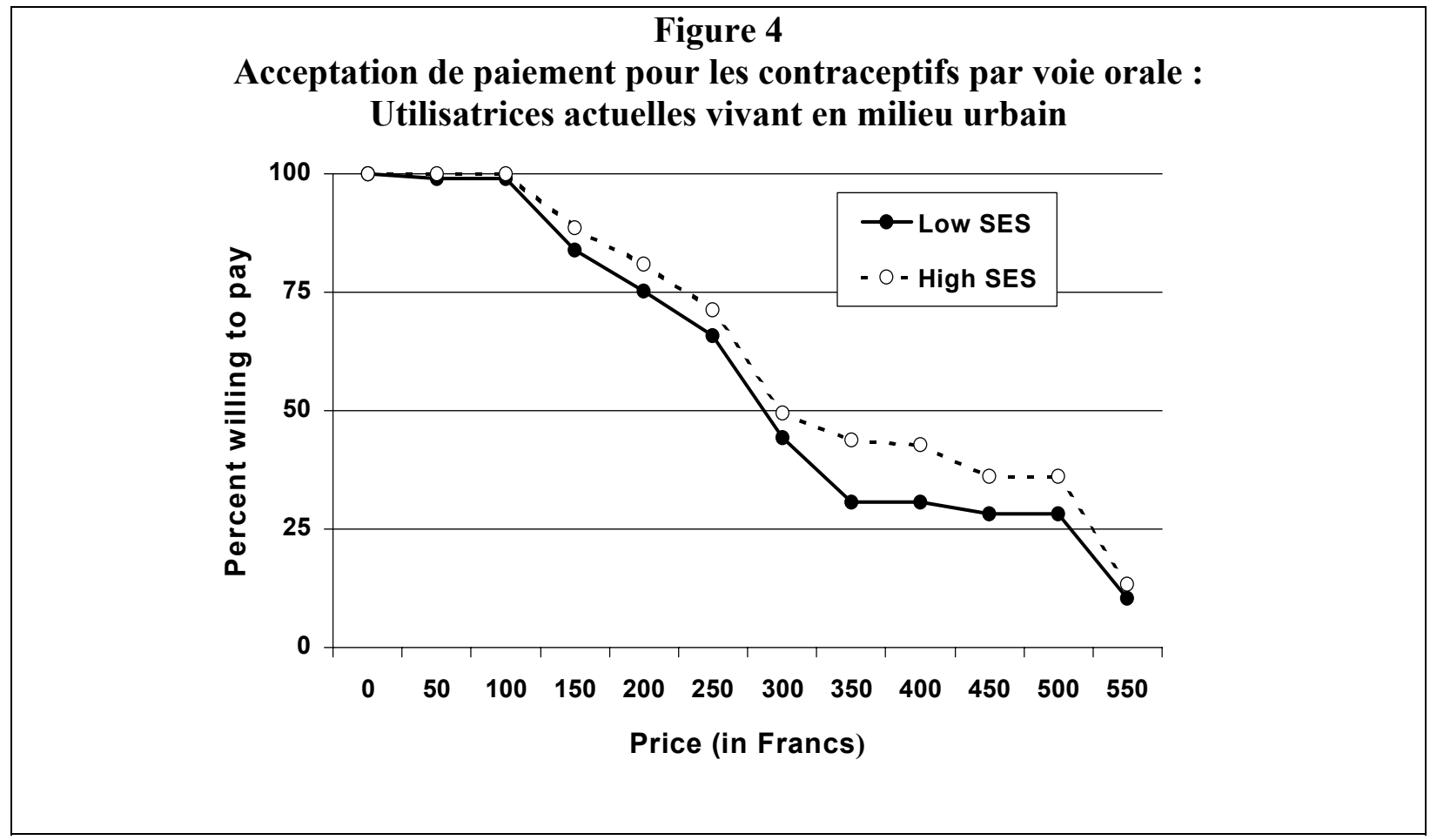

Percent willing to pay - Pourcentage prêt à payer

Price (in Francs) - Prix en francs

Low SES - Catégorie socioéconomique basse

High SES - Catégorie socioéconomique élevée

\footnotetext{
${ }^{8}$ Des critères différents ont été utilisés pour les femmes vivant en milieu urbain et celles qui vivaient en zone rurale. La différence absolue entre les catégories est relativement faible ; la plupart des familles, même dans les zones urbaines du Mali seraient considérées comme étant assez pauvres.
} 
L'acceptation de paiement a aussi été évaluée parmi les utilisatrices potentielles de la pilule. Il s'agissait de femmes qui n'avaient actuellement pas recours aux contraceptifs par voie orale ou injectables, ni à une forme de contraception chirurgicale et qui avaient répondu qu'elles pourraient commencer à utiliser la pilule ou s'y remettre à l'avenir ; 736 femmes ont été classées comme utilisatrices potentielles.

Treize personnes interrogées seulement, soit $2 \%$ des utilisatrices potentielles, n'ont pas pu donner un prix qu'elles seraient prêtes à payer pour cette méthode. Soixante-neuf pourcent étaient prêtes à payer le prix le plus élevé demandé ( $250 \mathrm{FM}$ ) et 58 pourcent de celles-ci ont même indiqué un prix encore plus élevé en tant que maximum qu'elles étaient prêtes à payer. L'acceptation de paiement a été pratiquement identique aux niveaux inférieurs de prix, encore que l'acceptation ait enregistré une légère baisse aux niveaux de prix les plus élevés. Cependant, l'acceptation d'un paiement plus élevé par les utilisatrices potentielles est plus élevée chez les femmes vivant en milieu urbain que chez celles des zones rurales et, dans les zones urbaines, celles des couches socioéconomiques plus élevées sont davantage prêtes à payer plus que celles des couches inférieures, comme l'indique la Figure 5.

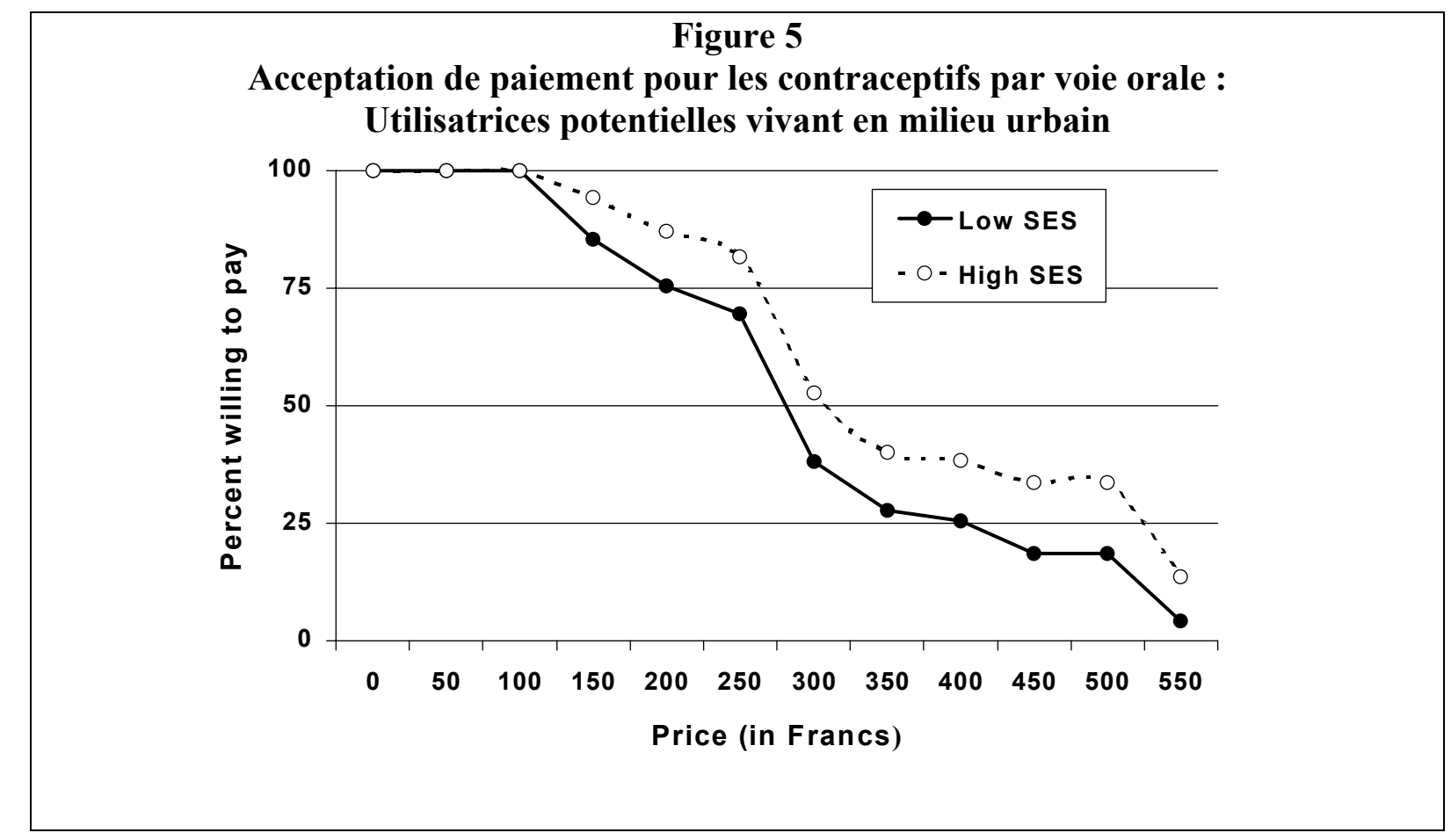

Percent willing to pay - Pourcentage prêt à payer

Price (in Francs) - Prix en francs

Low SES - Catégorie socioéconomique basse

High SES - Catégorie socioéconomique élevée

La Figure 6 donne les recettes attendues de Pilplan à différents prix. Le total des ventes pour 1998 s'est monté à 761200 cycles, représentant 76 millions au prix actuel de 100 FM. Le prix le plus élevé mentionné directement dans les questions était de $250 \mathrm{FM}$. Même avec la perte attendue d'un tiers des clientes de Pilplan, ce chiffre représente une recette potentielle de 128 millions de FM, soit une augmentation de 68 millions de FM par rapport aux recettes actuelles. En d'autres termes, les pertes 
attendues au niveau des ventes seraient plus que compensées par l'augmentation du prix. La ligne en pointillé de la Figure 6 représente les prix pour lesquels les estimations d'acceptation de paiement ne sont plus fiables.

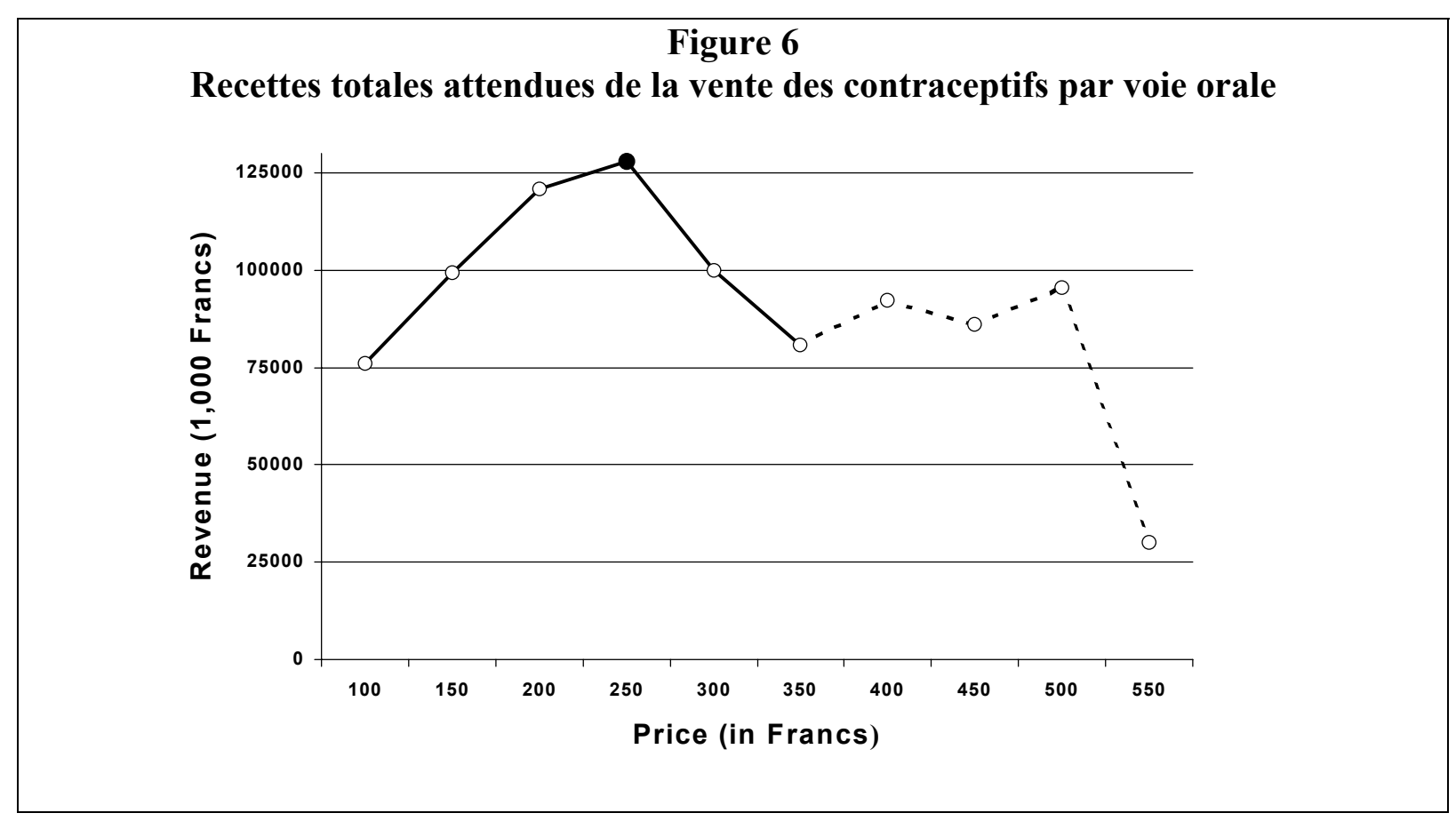

Revenue - Recettes (1 000 francs)

Price (in Francs) - Prix en francs

\section{Préservatifs}

Les hommes ayant répondu qu'ils avaient utilisé un préservatif dans les trois mois avant l'entretien ont été classés comme utilisateurs actuels de préservatifs. Un total de 742 personnes interrogées, soit 27 pourcent de l'échantillon répondait à ce critère. La marque Protector était en tête sur le marché dans toutes les zones, représentant 90 pourcent des préservatifs utilisés plus récemment. Cependant, par rapport aux femmes qui prennent la pilule, les utilisateurs de préservatifs masculins ont fait preuve d'une moindre cohérence en ce qui concerne la marque et la source -25 pourcent des utilisateurs actuels ont répondu qu'ils se servaient souvent d'une autre marque ou faisaient appel à une source différente. Cette différence entre les méthodes n'est pas surprenante étant donné que l'usage des préservatifs peut être moins constant que celui de la pilule. Lorsqu'on leur a demandé avec qui ils avaient utilisé un préservatif pour la dernière fois, 46 pourcent des hommes interrogés ont répondu par « une partenaire occasionnelle », y compris 36 pourcent des hommes mariés et 47 pourcents de ceux qui vivaient en cohabitation. Lorsqu' on leur a demandé pourquoi ils utilisaient des préservatifs, 73 pourcent des utilisateurs actuels n'ont mentionné que la prévention des maladies et 7 pourcent seulement la contraception. Les autres 20 pourcent ont mentionné la prévention des maladies aussi bien que de la grossesse.

Peu de personnes interrogées ont eu des difficultés à répondre aux questions relatives aux prix actuel ou à l'acceptation d'un prix hypothétique. Huit pourcent ne connaissaient pas la marque ou le prix du préservatif utilisé pour la dernière fois ; la probabilité de connaissance de la marque ou du prix a été 
légèrement supérieure chez les hommes ayant été scolarisés que chez ceux qui ne l'avaient pas été. $(9,5 \%$ contre $4,9 \% ; p<0,07)$. Tous les utilisateurs actuels ont pu répondre aux questions relatives aux prix hypothétiques. Soixante-neuf pourcent ont accepté l'augmentation de prix la plus élevée et 81 pourcent d'entre eux ont mentionné un prix encore plus élevé qu'ils étaient prêts à payer. Sept pourcent ont mentionné un prix qu'ils étaient prêts à payer qui était inférieur au prix le plus élevé qui avait été convenu. Bien que les niveaux aient généralement été bas, la scolarisation a joué un rôle dans la convergence des réponses : 12 pourcent des hommes n'ayant pas été scolarisés ont donné des réponses non convergentes, contre $6 \%$ pour ceux qui avaient été scolarisés ( $\mathrm{p}<0,01)$.

Un total de 873 hommes, soit 43 pourcent, de ceux qui n'avaient pas utilisé de préservatif au cours des trois derniers mois ont déclaré qu'ils seraient prêts à s'en servir à l'avenir. Moins de un pourcent de ces utilisateurs potentiels n'ont pas pu donner un prix qu'ils étaient prêts à payer. Soixante-dix-neuf pourcent des utilisateurs potentiels de préservatifs ont accepté le prix demandé le plus élevé - 150 FM - et 71 pourcent d'entre eux ont donné un prix encore plus élevé qu'ils seraient prêts à payer.

Comme cela a été constaté chez les femmes vivant en milieu urbain et prenant la pilule, les utilisateurs de préservatifs visant en milieu urbain et faisant partie d'une couche socioéconomique plus élevée se sont constamment avérés être plus prêts à payer un prix plus élevé que ceux des couches inférieures, encore que la différence n'ait pas été très marquée. Ces conclusions sont illustrées à la Figure 7. Parmi les utilisateurs potentiels de préservatifs, les hommes vivant en milieu urbain étaient davantage prêts à payer que ceux qui vivent en zone rurale et, parmi les utilisateurs potentiels vivant en milieu urbain, il y a eu une différence relativement substantielle dans ce domaine entre ceux d'un milieu socioéconomique supérieur et ceux d'un niveau inférieur. Il y a eu une prévision de demande plus élevée chez les utilisateurs potentiels de préservatifs à une gamme de prix plus bas que chez les utilisateurs actuels, ce qui a été une constatation inattendue.

\section{Figure 7 \\ Acceptation de paiement pour les préservatifs : Utilisateurs actuels - Hommes vivant en milieu urbain}

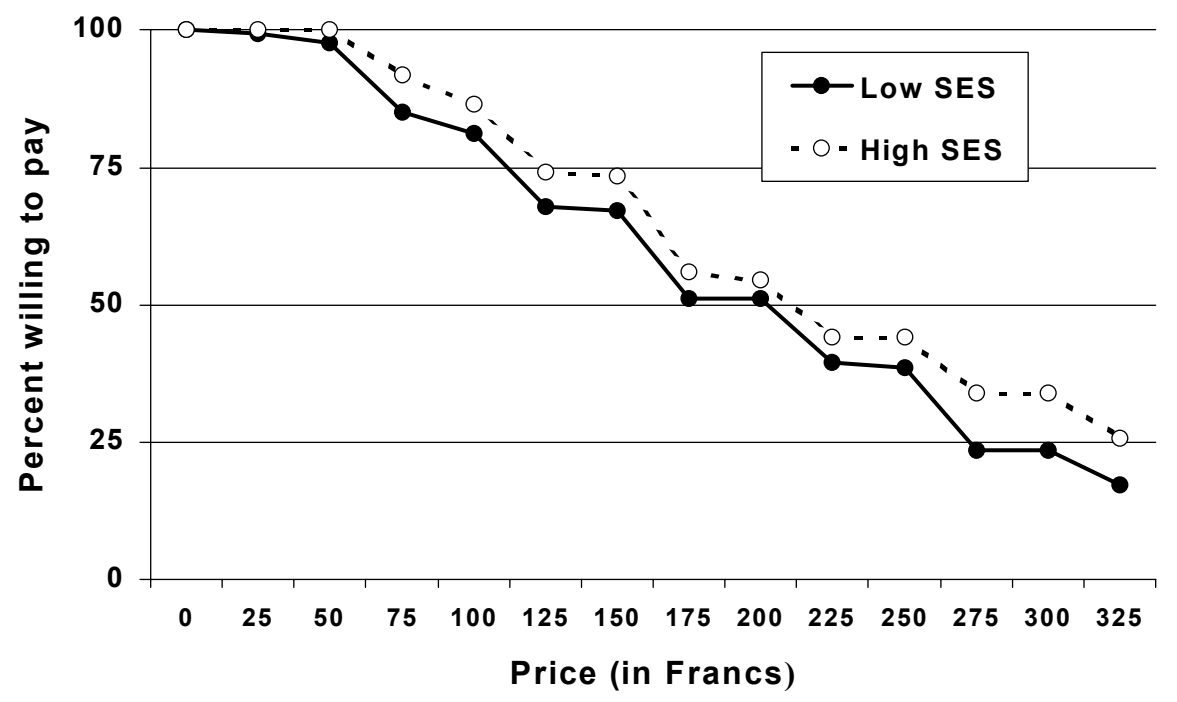


Percent willing to pay - Pourcentage prêt à payer

Price (in Francs) - Prix en francs

Low SES - Catégorie socioéconomique basse

High SES - Catégorie socioéconomique élevée

Comme cela avait été constaté pour les contraceptifs par voie orale, le point des recettes potentielles les plus élevées pour les contraceptifs correspondait aussi au prix le plus élevé qui avait été mentionné 150 FM pour un paquet de trois préservatifs. La Figure 8 présente la courbe des recettes anticipées pour la marque Protector. Il faut noter que les recettes feraient plus que doubler si les prix étaient augmentés de 50 à 150 FM, passant de 85 à 176 millions de FM. Comme dans le cas de la Figure 6, la ligne en pointillé représente les prix pour lesquels les estimations d'acceptation de paiement ne sont plus fiables.

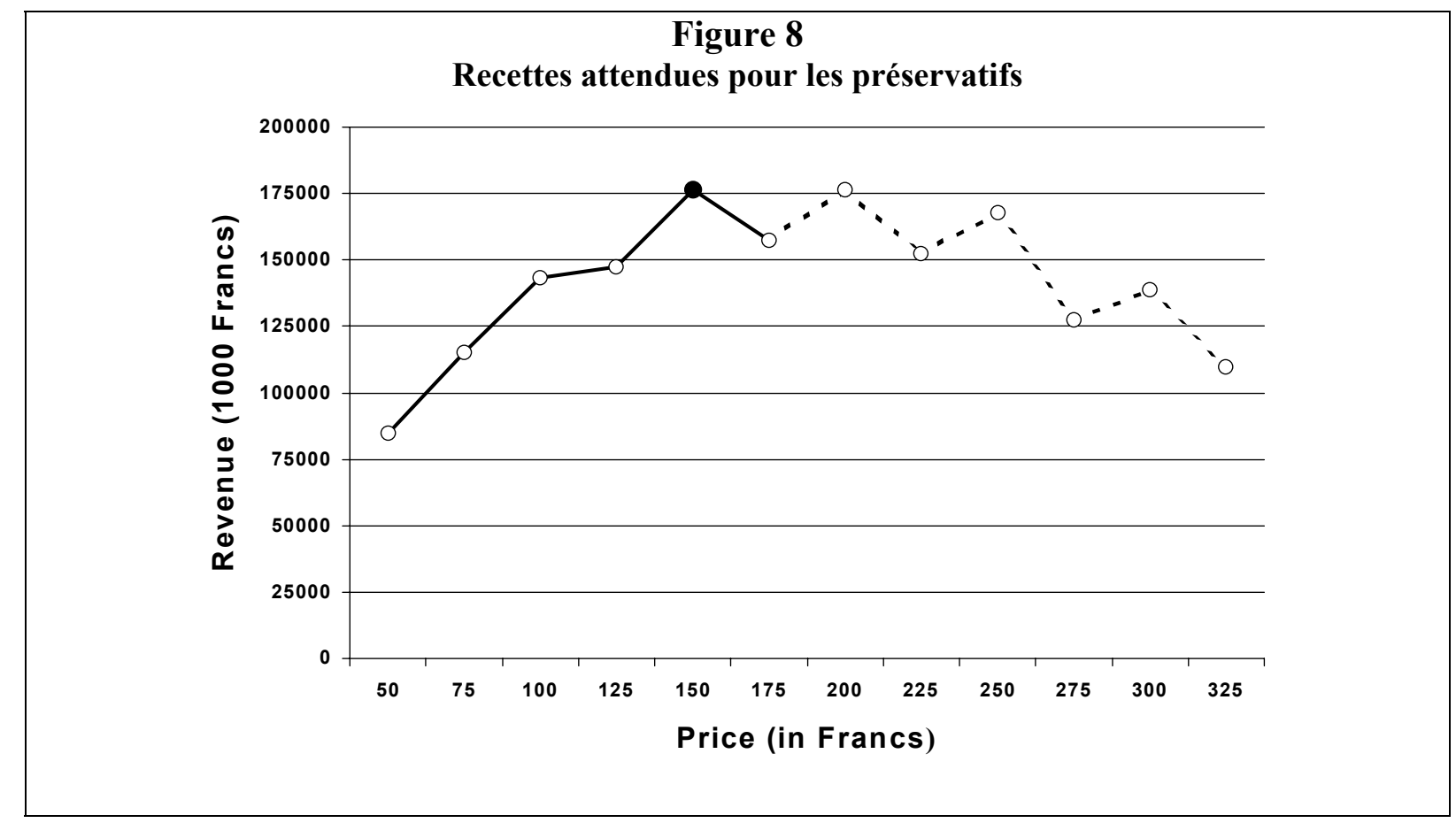

Revenue (1000 Francs) - Recettes (1 000 francs)

Price (in Francs) - Prix en francs

Que pouvons-nous conclure de l'application au Mali de la méthodologie d'acceptation de paiement?

1. Même dans une région où le niveau d'alphabétisation est bas, les utilisateurs actuels de divers moyens de contraception peuvent comprendre des questions sur ce qu'ils pourraient faire sous des conditions hypothétiques différentes et y répondre de façon cohérente. Pratiquement toutes les personnes interrogées - les utilisateurs aussi bien actuels que potentiels — ont pu répondre par oui ou par non à la première question hypothétique relative aux prix.

2. Demander aux personnes interrogées quel est le prix maximum qu'elles seraient prêtes à payer étend nettement la fourchette des prix potentiels parmi celles qui acceptent le prix le plus élevé mentionné dans les questions. Il est probable que celles qui refusent un ou plusieurs de ces derniers ne donneront 
pas un prix maximum plus élevé que le dernier qu'elles ont accepté. Poser la question relative au prix maximum permet en outre de vérifier si la personne interrogée a bien compris les questions

hypothétiques. Peu de personnes interrogées donnent des estimations qui ne sont pas convergentes en réponse à cette question, encore que celles qui n'ont pas été scolarisées ont davantage de chance de faire preuve de confusion que celles qui l'ont été.

3. La validité interne a été généralement élevée ; les personnes interrogée faisant partie d'une couche socioéconomique plus élevée se sont montrées davantage prêtes à payer que celles des couches inférieures et, parmi les utilisateurs potentiels, les personnes interrogées vivant en milieu urbain l'acceptation de paiement a été plus élevée que pour celles des zones rurales.

\section{Pakistan : Détermination de prix de produits nouveaux}

Déterminer les prix de produits nouveaux dans le cadre du marketing social pose plusieurs problèmes. Premièrement, il n'existe pas de données permettant de guider la prise de décisions car le produit est nouveau sur le marché. Deuxièmement, même dans le cas ou il existe des produits ou services similaires dans les secteurs public ou privé, il se peut que le nouveau produit soit tellement différent au niveau de sa posologie, de son monde d'administration et ainsi de suite que les informations disponibles ne permettent pas une comparaison utile. Les données WTP peuvent aussi fournir des informations utiles dans une telle situation, ce qu'a démontré la recherche effectuée au Pakistan.

Le Projet pour la population du secteur privé du Pakistan (PPSPP) a été lancé dans deux villes :

Faisalabad and Larkana. Le projet portait sur des contraceptifs administrés par voie orale ou par injection, à des prix subventionnés inférieurs à ceux des produits entièrement commerciaux. Des enquêtes WTP ont été effectuées en août 1996 dans les deux villes ciblées. En raison des préoccupations exprimées par le gouvernement du Pakistan concernant la question des prix des contraceptifs dans le secteur public des zones rurales, une de ces dernières a été ajoutée à l'échantillonnage.

Le marché ciblé par le PPSPP était les couples mariés dont le revenu mensuel allait de 1500 à 4000 roupies (entre 43 et 113 dollars). On pensait que les personnes probablement prêtes à accepter ces produits étaient les femmes de moins de 40 ans ayant déjà au moins un enfant vivant. L'échantillonnage destiné à l'enquête WTP a été déterminé à partir des districts électoraux des zones urbaines de Faisalabad et de Larkana, où la majorité des personnes interrogées tombaient dans la fourchette de revenu.

L'échantillon rural a été choisi parmi des villages se trouvant à entre cinq et 25 kilomètres des limites de Faisalabad, où il y avait davantage de chances de trouver des personnes dans la même tranche de revenu. Seules les femmes répondant aux critères d'âge et de revenu ont été interrogées à propos de quatre prix $25,15,10$ et 3 roupies - en ordre décroissant. Les techniques de détermination de l'acceptation de paiement étaient toujours en cours d'élaboration à cette époque et le questionnaire ne comportait pas la question relative au prix maximum.

Un échantillon total de 1753 femmes a été interrogé. Deux pourcent seulement de celles-ci utilisaient alors des contraceptifs par voie orale, mais 6 pourcent ont déclaré qu'elles pourraient envisager de le faire à l'avenir. Les femmes pour lesquelles la pilule était le moyen de contraception préféré ont été classées comme étant fortement motivée à utiliser cette méthode alors que celles dont le moyen de contraception était différent ont été classées comme l'étant peu. La force motivationnelle a été clairement un facteur d'acceptation de paiement : les femmes préférant la pilule étaient davantage prêtes à accepter de payer n'importe quel prix indiqué que les autres, comme on peut le constater à la Figure 9. 


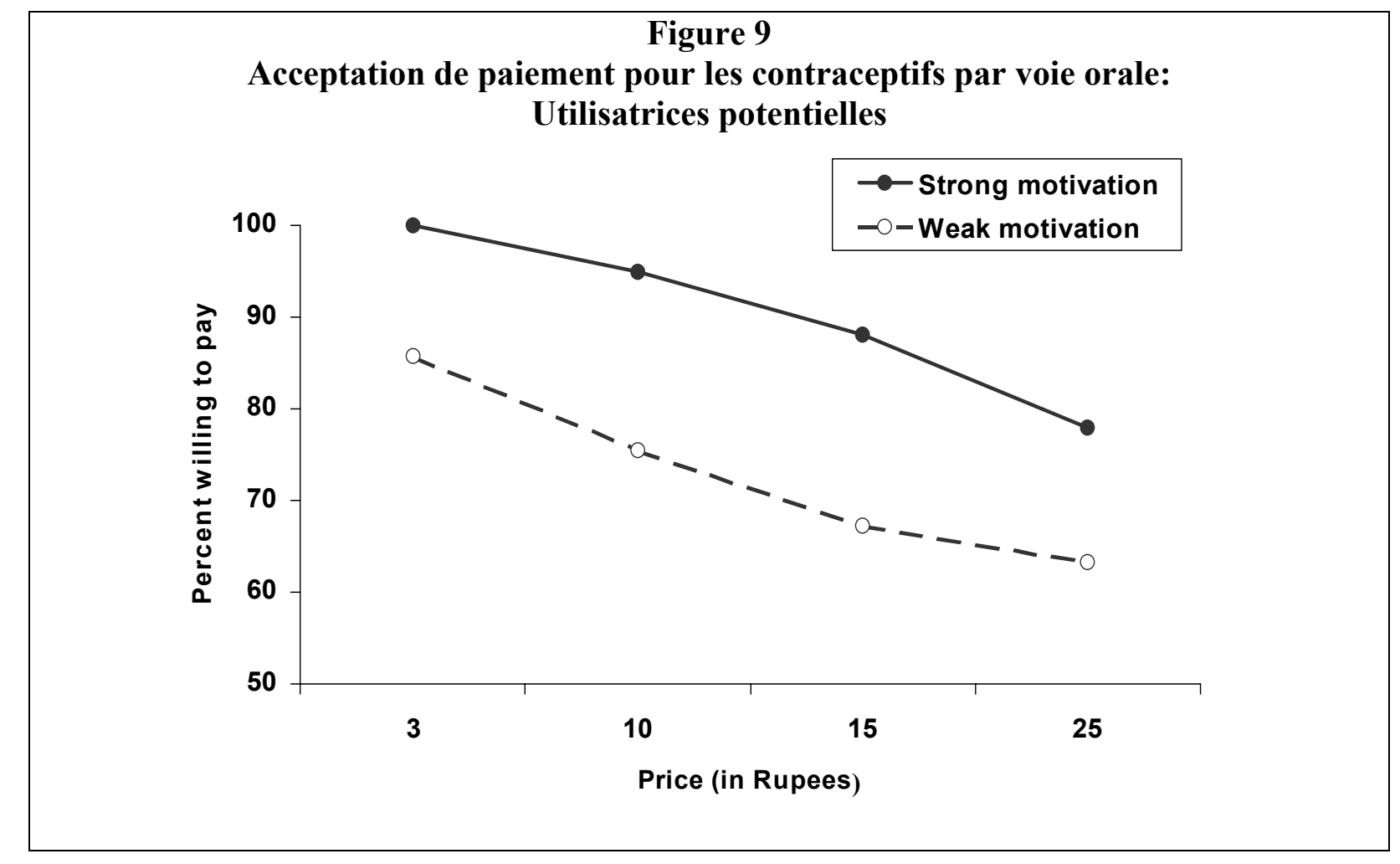

Percent willing to pay - Pourcentage prêt à payer

Price (in Rupees) - Prix en roupies

Strong motivation - Forte motivation

Weak motivation - Faible motivation

A la suite de l'enquête WTP, le prix de la marque faisant l'objet du marketing social a été fixé à 15

roupies par cycle, montant que 88 pourcent des femmes les plus prêtes à les acheter se sont dites prêtes à payer.

A la différence de l'étude effectuée au Mali, qui avait été conçue pour tester l'acceptation de paiement de produits existants, celle du Pakistan avait pour but de donner des informations destinées à fixer le prix de produits nouveaux. Ce qui a été appris au Pakistan en matière de méthodologie renforce et étend ce qui avait été retenu au Mali, comme suit:

1. Les personnes n'utilisant pas le moyen en question peuvent répondre aux questions WTP. Une fois de plus, le taux de celles qui ont répondu par je ne sais pas a été très faible et les enquêtrices n'ont pas fait état de problèmes dans l'administration du questionnaire.

2. Les prix recommandés, provenant de l'estimation directe de l'acceptation de paiement, sont dans la gamme des critères utilisés couramment en ce qui concerne les capacités de paiement (exprimés en 
pourcentage du revenu familial) et correspondent aux dépenses indiquées par les personnes interrogées dans le domaine de l'hygiène préventive. ${ }^{9}$

3. La validité interne de la méthodologie WTP a été confirmée : les femmes n'utilisant actuellement pas la pilule mais fortement intéressées par ce moyen étaient davantage prêtes à payer que les moins motivées.

\section{Equateur : Augmentation des prix des services ${ }^{10}$}

Les deux premières études de cas démontrent la convergence et la fiabilité de la technique WTP. Mais à quel point un directeur de programme peut-il être certain que les résultats d'une enquête WTP prédiront effectivement si ses clients sont prêts à payer un nouveau prix ? En d'autres termes, à quel point cette méthodologie est-elle valide ? L'étude de cas suivant tente d'apporter une réponse à cette question.

L'étude a été effectuée avec le Centros Medicos de Orientacíon y Planificacíon Familiar (Centre médical de conseils et de planning familial, connu sous son sigle en espagnol, CEMOPLAF), une ONG équatorienne qui fournit toute gamme de services dans le domaine de la santé de la reproduction. Les clients du CEMOPLAF sont surtout des femmes de la classe ouvrière vivant en milieu urbain, dont 20 pourcent vivent au niveau du seuil de pauvreté ou en dessous. Pour tenir compte de l'inflation, le CEMOPLAF augmente périodiquement ses prix d'environ 20 pourcent. Malgré ces hausses et des mesures destinées à réduire les coûts et à attirer davantage de clients, le CEMOPLAF avait besoin d'accroître ses recettes et avait décidé en 1996 de tester des augmentations de prix supérieures aux 20 pourcent habituels.

Quinze des 21 dispensaires du CEMOPLAF desservant une clientèle ayant le même profil socioéconomique ont pris part à une expérience portant sur trois augmentations de prix différentes, précédées par une enquête WTP. ${ }^{11}$ Les changements intervenant dans le recours au dispensaire à la suite de la hausse des prix ont été comparés à ceux qui avaient été prévus par l'enquête.

L'enquête de référence a été effectuée deux mois avant l'entrée en vigueur prévue de la hausse des prix. Il a été demandé aux clientes des dispensaires arrivant pour une visite d'obstétrique/gynécologie, de pose de stérilet et de soins prénatals à quel point elles seraient prêtes à payer des augmentations hypothétiques des prix de ces services. Comme dans le cas du Mali, on leur a d'abord proposé une augmentation modérée (de 4500 sucres, soit 1,37 dollar), qui a ensuite été relevée ( 9000 sucres, soit 2,74 dollars) ou réduite ( 2500 sucres, soit 0,76 dollar) en fonction de la réponse à la première augmentation proposée. Il a ensuite été demandé aux personnes ayant accepté l'augmentation la plus forte quel prix elles étaient prêtes à payer.

\footnotetext{
${ }^{9} \mathrm{La}$ 《sagesse populaire » suggère que les prix des produits faisant l'objet d'un marketing social en matière de contraception devraient représenter entre 1 et 3 pourcent du revenu des utilisatrices ciblées. A 15 roupies par cycle, une année de contraceptifs (195 roupies : 15 x 13 cycles) constitue 1,3 pourcent ou moins du revenu mensuel du segment du marché ciblé (entre 1500 et 4000 roupies). Parmi les femmes potentiellement intéressées par la contraception par voie orale, les dépenses annuelles moyennes par ménage pour l'hygiène préventive se montaient à 300 roupies, soit la moitié du prix d'une année du prix annuel du moyen de contraception. Le prix de trois mois de préparation injectable a été fixé à 60 roupies, ce qui représente au maximum 1,6 pourcent du revenu annuel du ménage pour une année de contraception; 80 pourcent des utilisatrices potentielles se sont dites prêtes à payer ce prix.

${ }^{10}$ Pour les détails de cette étude, voir de Vargas et al. (1998).

${ }^{11}$ Une analyse de groupage a été effectuée afin d'identifier les dispensaires desservant une clientèle aux caractéristiques socioéconomiques similaires. Elle a été basée sur quatre variables : pourcentage de clients ayant un emploi rémunéré en dehors du foyer, pourcentage de personnes possédant un réfrigérateur, pourcentage de personnes ayant consommé de la viande au moins deux des trois jours précédents et revenu familial moyen (voir Bratt et al. 1998).
} 
Un total de 3661 patientes de dispensaires ont été interrogées, dont environ un tiers venaient pour une pose de stérilet, plus de la moitié pour une consultation gynécologique et environ 12 pourcent pour un examen prénatal. En tout, 79 pourcent ont accepté l'augmentation de prix moyenne et 53 pourcent la hausse la plus forte. Il n'y a pas eu de différence entre les services. Qui plus est, 59 pourcent de celles qui avaient accepté l'augmentation la plus forte se sont dites prêtes à payer un prix maximum encore plus élevé.

La Figure 10 présente l'acceptation de paiement pour ces trois services de clinique. Il faut noter que la prévision de la demande concernant les services de gynécologie et de soins prénatals est pratiquement identique et que celle des visites pour pose de stérilet est parallèle, mais légèrement plus faible. Le CEMOPLAF demande des prix plus élevés pour les services de gynécologie et prénatals afin de subventionner ceux de pose de stérilet ; le prix moyen initial des services de gynécologie et prénatals était de 8000 sucres, par rapport à 6500 pour ceux de pose de stérilet.

Figure 10

Acceptation de paiement pour les services de clinique

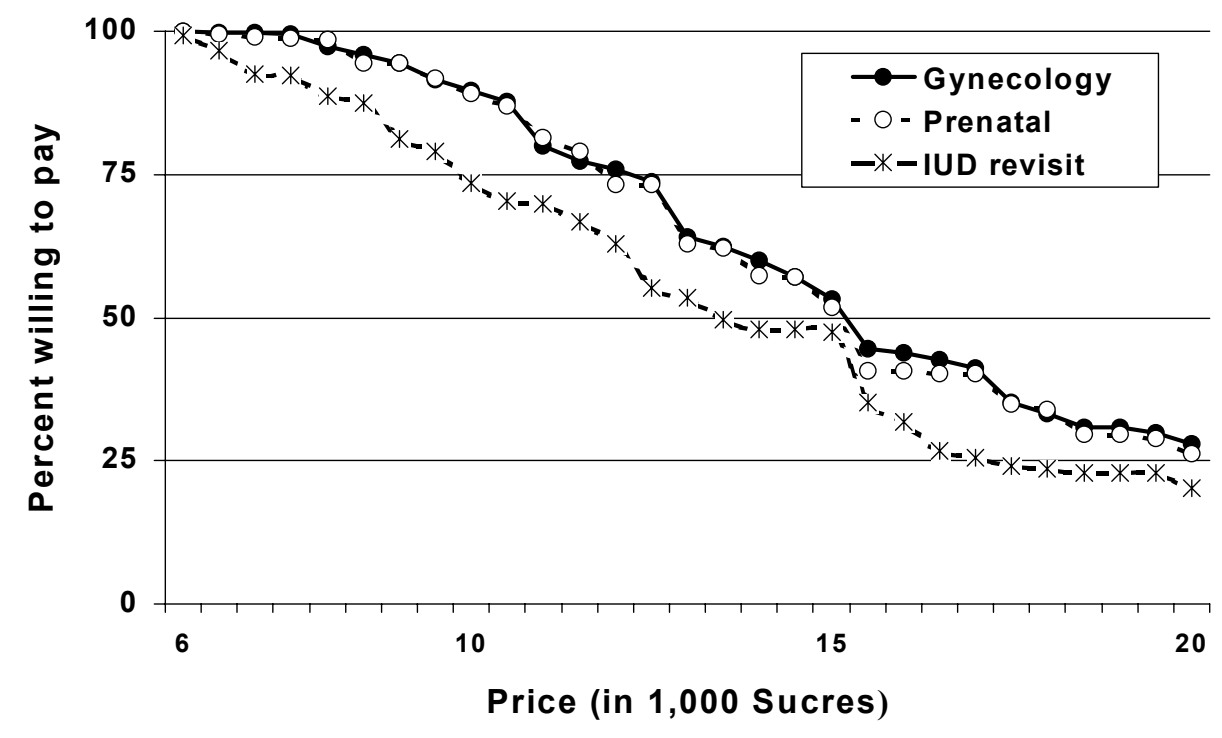

Percent willing to pay - Pourcentage prêt à payer

Price (in 1,000 Sucre) - Prix (1 000 sucres)

Gynecology - Gynécologie

Prenatal - Soins prénatals

IUD revisit - Examen stérilet

Validité de prédiction. Deux mois après l'enquête WTP, les 15 dispensaires qui y avaient pris part ont appliqué de façon aléatoire différentes augmentations de prix $(20 \%, 40 \%$ ou $60 \%)$ pour les visites de pose de stérilet, les consultations gynécologiques et les examens prénatals. Le volume mensuel moyen des 
visites des trois mois ayant précédé l'enquête a été comparé au volume mensuel moyen de celles qui ont suivi l'augmentation des prix.

La Figure 11 présente la demande prévue et enregistrée pour ces trois services de clinique à la suite du changement des prix. ${ }^{12}$ Il faut noter à quel point la demande prévue et celle qui a été constatée pour les visites de pose de stérilet et gynécologiques est proche. Pour ces deux services, la demande constatée a baissé à la suite de l'augmentation de prix, mais les différences entre services n'ont pas été statistiquement fiables. Les prévisions d'acceptation de paiement ont été moins précises pour les services prénatals et, paradoxalement, l'augmentation la plus forte de la demande à la suite de la hausse des prix a été enregistrée pour l'augmentation la plus élevée. Là encore, il n'a pas été possible d'obtenir une fiabilité statistique dans les différences observées entre prix.

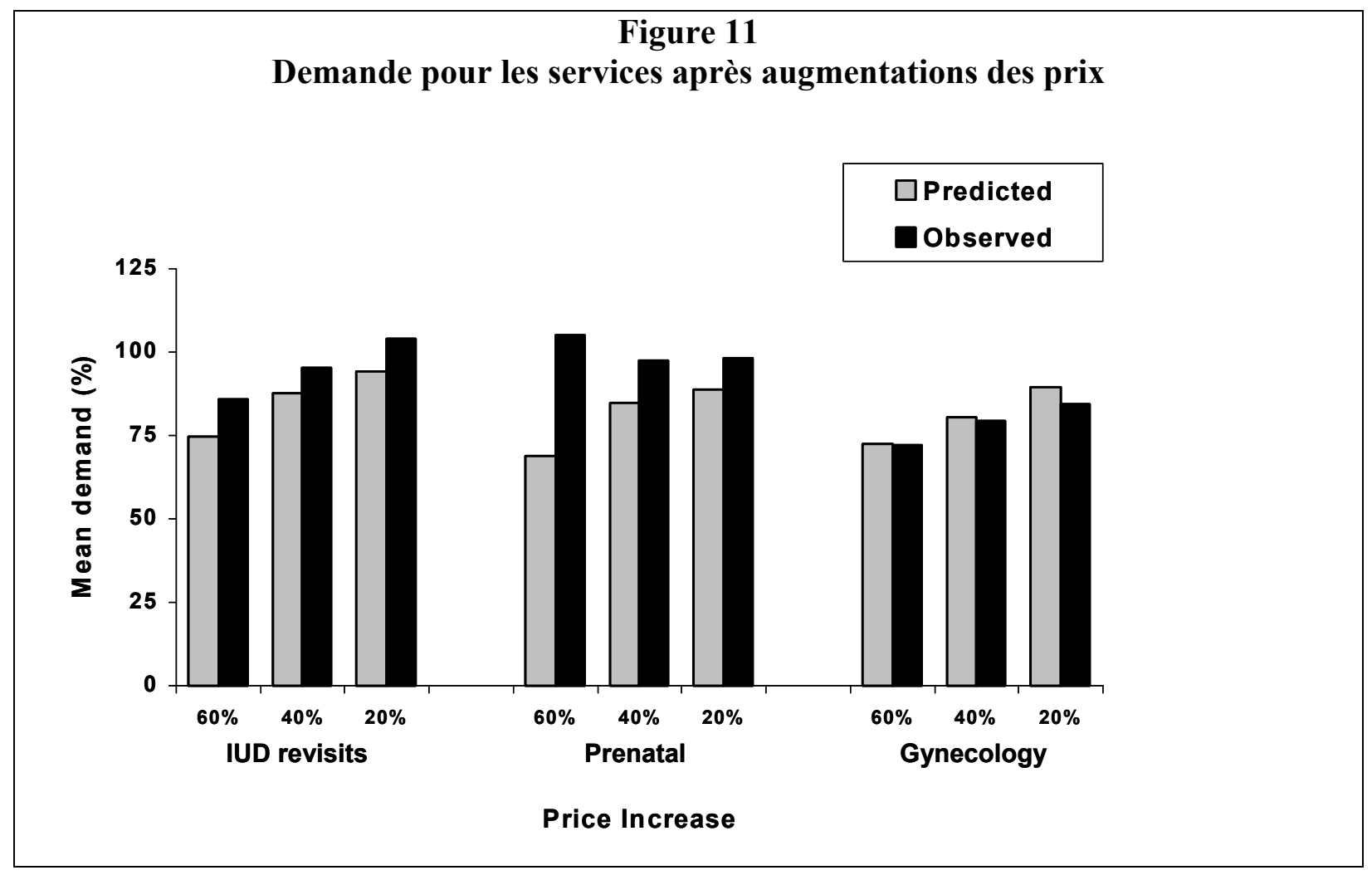

Mean demand (\%) - Demande moyenne (\%)

Predicted - Prévue

Observed - Observée

IUD revisits - Examen stérilet

Prenatal - Soins prénatals

Gynecology - Gynécologie

Price Increase - Augmentation des prix

\footnotetext{
${ }^{12}$ Les courbes de demande globale indiquées à la Figure 10 ont servi à calculer la demande pour chaque service, dans chaque dispensaire, au niveau prix, à l'aide de la méthode décrite à la Section III. La Figure 11 présente la demande prévue en tant que pourcentage du volume de référence (pourcentage de la demande globale au nouveau prix, divisé par le pourcentage de la demande globale au prix original).
} 
L'analyse du volume de la clientèle (nombre de consultations) à la suite de l'augmentation des prix a indiqué que le pourcentage de changement prévu était de \pm 5 pourcent de celui qui a été constaté dans 12 des 44 combinaisons dispensaire/service ( $27 \%$ ) et de l'ordre de \pm 10 pourcent des changements observés dans 21 cas (48\%). Finalement, les prévisions d'acceptation de paiement ont surestimé les pertes de clientes (c'est-à-dire que les pertes prévues ont été plus importantes que celles qui ont été constatées) dans 68 pourcent des cas. Cette perte a été sous-estimée de moins de 5 pourcent dans 14 pourcent supplémentaires des cas. La Figure 12 donne la ventilation des pertes sur et sous-estimées en clientes, prouvant que la technique d'estimation directe contribue à protéger les programmes contre la sousestimation des pertes de clients.

\section{Figure 12 \\ Précision des prévisions WTP}

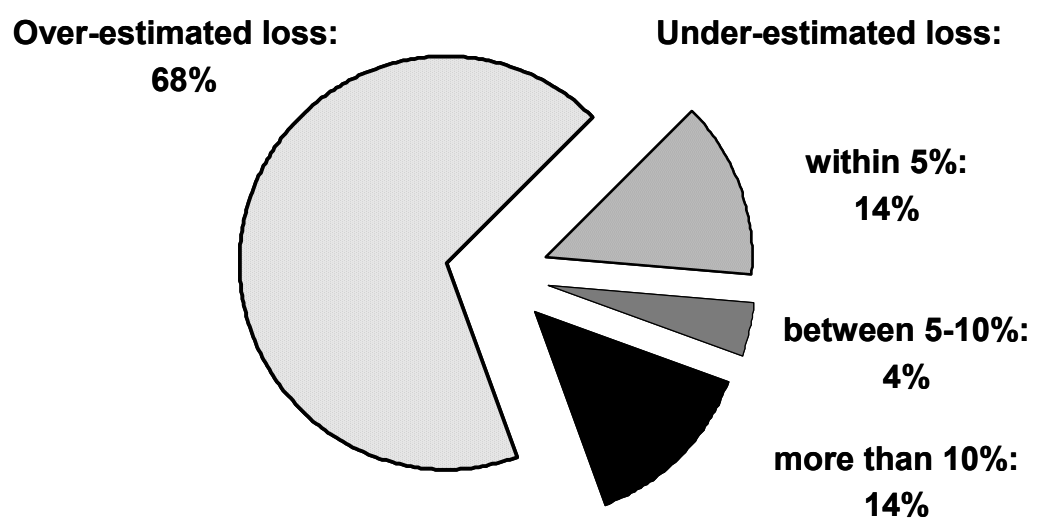

Over-estimated loss : 68\% - Pertes surestimées : $68 \%$

Under-estimated loss : Pertes sous-estimées

within 5\%:14\% - Moins de $5 \%: 14 \%$

between $5-10 \%: 4 \%$ - Entre 5 et $10 \%: 4 \%$

more than $10 \%: 14 \%$ - Plus de $10 \%: 14 \%$

Les estimations d'acceptation de paiement sont un meilleur prédicteur du volume de services après hausse des prix que toutes les autres variables dont les directeurs de programmes disposent, comme le volume initial de service ou l'amplitude de la hausse des prix. L'analyse de variance avec régression hiérarchique multiple a été utilisée en tant que moyen prudent de tester la validité de prédiction, en saisissant d'abord la volume de référence, l'augmentation de prix et enfin des prévisions d'acceptation de paiement. Le volume futur a été presque parfaitement en corrélation avec le volume de référence, ce qui n'est pas surprenant $\left(\mathrm{R}^{2}=0,93\right)$. Cependant, les prévisions WTP ont nettement amélioré celles du changement par rapport aux niveaux de référence, de $\mathrm{R}^{2}=0,095$ pour le volume de référence à lui seul à $\mathrm{R}^{2}=0,331$. Les augmentations de prix n'ont jamais prévu la performance future, quelle que soit la variable dépendante. 
L'étude effectuée en Equateur renforce les leçons apprises au Mali et au Pakistan et ajoute une méthodologie pour la validation expérimentale des estimations tirées de questionnaires WTP :

1. Les clientes des dispensaires n'ont pas eu beaucoup de problème à répondre à des questions portant sur des augmentations de prix hypothétiques ou le prix maximum qu'elles étaient prêtes à payer. L'inclusion de la question relative au prix maximum a étendu la fourchette des augmentations potentielles de prix sans beaucoup surcharger le questionnaire.

2. La validation expérimentale de trois augmentations de prix a été conforme à la prévision d'une baisse de la demande à la suite d'une augmentation de prix. Les estimations WTP améliorent nettement la capacité des directeurs de programmes à prévoir l'impact des changements de prix. La tendance systématique des enquêtes WTP à surestimer la baisse de la demande à la suite d'une hausse des prix fait qu'elles sont particulièrement utiles pour la détermination de prix dans le secteur à but non lucratif dans lequel la nécessité de maintenir le niveau de volume impose l'utilisation de méthodes d'estimation prudentes. 


\section{Test de la validité de prédiction}

A ce jour, la seule validité de prédiction des enquêtes WTP dans le domaine de la santé de la reproduction à avoir été publiée est l'étude du CEMOPLAF en Equateur. Il faut effectuer davantage de tests de validité de prédiction. Mais différentes applications WTP requièrent différentes considérations méthodologiques.

Test des augmentations de prix pour utilisateurs actuels de produits ou services. On peut utiliser une de deux approches en fonction du service ou du produit considéré. La première consiste à utiliser les données relatives aux clients actuels pour prévoir le comportement de futurs clients. Elle part de l'hypothèse que les uns et les autres proviennent de la même population et ont une capacité et une acceptation de paiement similaires. Dans un tel cas, la validité de prédiction est évaluée en suivant les étapes suivantes : 1) des informations de référence sur l'utilisation sont collectées, le plus souvent en faisant appel aux statistiques relatives au service ou aux données de vente ; 2) une enquête WTP est effectuée pour obtenir des données sur l'utilisation prédite ; 3 ) les prix sont augmentés de façon aléatoire dans des sous-groupes de dispensaires (la validation par analyse de régression multiple, comme dans le cas de l'étude du CEMOPLAF, requiert au moins deux niveaux de prix); et 4) la précision de la prévision est comparée à l'utilisation constatée, qui est mesurée par les données statistiques relatives au service après la hausse des prix. Il est possible de maximiser le nombre des constatations en utilisant des combinaisons dispensaire/service en tant qu'unité d'analyse. (Par exemple, si les prix doivent être augmentés pour quatre services dans 10 dispensaires, le nombre de constatations est de $4 \times 10=40$.) Il faut que le chercheur utilise un aussi grand nombre de cas que possible car les programmes sont très " surchargés » de nombreuses variables. Le facteur saisonnier intervient souvent aussi bien au niveau des dispensaires que des services et ses dates peuvent varier en fonction de chaque dispensaire ou service. Il se peut que certains dispensaires aient une trajectoire caractérisée par une utilisation accrue à terme alors qu'elle est en baisse ou plate pour d'autres.

Il existe une seconde possibilité d'évaluation de la validité de prédiction des enquêtes WTP lorsque l'on peut suivre le comportement des utilisateurs individuels sur un certain temps. Le test le plus rigoureux de la validité de prédiction d'une enquête donnée consiste à comparer ses résultats au comportement ultérieur des personnes interrogées. Par exemple, Griffin et al. (1995) ont demandé aux personnes interrogées en Inde si elles seraient prêtes à payer un droit de branchement et des frais mensuels pour un meilleur système d'approvisionnement en eau. Les ménages ont été à nouveau interrogés une fois le nouveau système en place. Quatre-vingt-onze pourcent des familles ayant déclaré en 1988 qu'elles s'y brancheraient l'ont fait en 1991 et 94 pourcent de celles ayant répondu non ne l'ont pas fait. Il est rare que l'on puisse se livrer à des études longitudinales dans le domaine de la santé de la reproduction, en partie en raison du temps et des moyens requis et en partie parce que nombre de ses produits et services ne sont pas utilisés constamment à relativement long terme. Par exemple, les clientes des services prénatals ne reviendront pas au dispensaire une fois que leurs enfants sont nés et une personne utilisant un stérilet peut n'avoir besoin que de quelques rares examens pendant les longues années d'utilisation de cette méthode.

Détermination des prix pour les produits ou services nouveaux. Il semble que la validité de prédiction des techniques WTP n'ait jamais été testée dans le cadre de la détermination de prix de nouveaux produits ou services. Il peut être possible, dans certaines situations, de faire appel à une approche longitudinale, comme celle qui a été mentionnée par Griffin et al. (1995). Mais cela ne sera pas possible pour la plupart des produits et services. Parmi les problèmes à résoudre avant de pouvoir valider la validité de prédiction des enquêtes WTP pour des nouveaux produits, on peut citer le manque de données de référence aux fins de comparaison et le fait que l'adoption d'un nouveau produit ou service peut être influencée par un grand nombre de facteurs autres que son prix. 


\section{Bibliographie}

Aker, B., M. Boroush et S. Smith. 1989. "The Choice to Contracept in Liberia: Sensitivities to Changes in Incomes and Contraceptive Prices" (Le choix d'adopter la contraception au Liberia : sensibilités aux changements intervenant dans les revenus et les prix des contraceptifs). Rapport SOMARC. Washington D.C. : The Futures Group International.

Akin, John S. et Brad Schwartz. 1988. "The Effect of Economic Factors on Contraceptive Choice in Jamaica and Thailand: A Comparison of Mixed Multinomial Logit Results" (Les effets des facteurs économiques sur le choix des contraceptifs à la Jamaïque et en Thaïlande : Une comparaison de résultats de logit multinominal mixte). Economic Development and Cultural Change 36(3): 503-527.

Bratt, John H., James Foreit et Teresa de Vargas. 1998. "Three Strategies to Promote Sustainability of CEMOPLAF Clinics in Ecuador" (Trois stratégies de promotion de la durabilité des cliniques CEMOPLAF en Equateur). Studies in Family Planning 29(1): 58-68.

Foreit, J.R. et Foreit, K.G. 2003. "The reliability and validity of willingness to pay surveys for reproductive health pricing decisions in developing countries" (La fiabilité et la validité des enquêtes d'acceptation de paiement dans les décisions de détermination des prix en matière de santé de la reproduction dans les pays en développement). Health Policy 63 (1): 37-47.

Griffin, C., J. Briscoe, B. Singh, R. Ramasubban et R. Bhatia. 1995. "Contingent Valuation and Actual Behavior: Predicting Connections to New Water Systems in the State of Kerala, India" (Evaluation conditionnelle et comportement réel : Prévoir les raccordements au nouveau système de distribution de l'eau de l'état de Kerala, en Inde). The World Bank Economic Review 9(3): 373-395.

Jensen, Eric R., N. Kak, K. Satjawinata, D.N. Wirawan, N. Nanjoy et Suproyoko. 1993. "Contraceptive Pricing and Prevalence: Family Planning Self-Sufficiency in Indonesia" (Prix et prévalence des contraceptifs : Autosuffisante du planning familial en Indonésie), document soumis à la réunion annuelle de l'Association américaine pour la population, Cincinnati, Ohio.

Leon, Federico R. et Augustin Cuesta. 1993. "The Need for Quasi-Experimental Methodology to Evaluate Pricing Effects" (La nécessité d'avoir une méthodologie quasi-expérimentale pour évaluer les effets de l'établissement des prix). Studies in Family Planning 24(6), part 1:375-381.

Lewis, Maureen A. 1986. "Do Contraceptive Prices Affect Demand?" (Les prix des contraceptifs affectentils la demande ?) Studies in Family Planning (17)3: 126-135.

Etudes du National Institute of Population et d'IRD/Macro International. 1992. Pakistan Demographic and Health Survey (Enquête de démographie et de santé - Pakistan) 1990/1991. Columbia, MD : National Institute of Population Studies (Pakistan) et IRD/Macro International Inc.

Oliver, Raylynn. 1994. "The Effect of the Quality, Price, and Availability of Family Planning on Contraceptive Use in Ghana" (Les effets de la qualité, des prix et de la disponibilité du planning familial sur son utilisation au Ghana). Document de travail de mesure de la qualité de la vie, Banque mondiale, départements Technique et Recherche politique pour l'Afrique. Washington, D.C. : Banque mondiale.

Phillips, Kathryn A., Rick K. Homan, Harold S. Luft, Patricia H. Hiatt, Kent R. Olson, Thomas E. Kearney et Stuart E. Heard. 1997. "Willingness to Pay for Poison Control Centers" (Acceptation de paiement pour les centres anti-poison). Journal of Health Economics 16: 347. 
Portnoy, Paul R. 1994. "The Contingent Valuation Debate: Why Economists Should Care" (Le débat sur l'évaluation conditionnelle : Pourquoi cela devrait intéresser les économistes). Journal of Economic Perspectives (8)4: 3-17.

de Vargas, Teresa, John H. Bratt, Varuni Dayaratna, James R. Foreit et Daniel H. Kress . 1998. "Estimating Consumer Response to Price Changes in Family Planning and Reproductive Health Services" (Estimer la réaction du consommateur aux changements de prix dans les services de planning familial et de santé de la reproduction), document soumis à la réunion annuelle de l'Association américaine pour la population, Chicago, Illinois. 


\section{ANNEXE I \\ QUESTIONNAIRES MODELES}

Modèle pour utilisateurs actuels et potentiels de services de clinique

(Entretien d'interception)

Enquête auprès des ménages sur les utilisateurs actuels ou potentiels de préservatifs

(Entretien au foyer) 


\section{Santé de la reproduction \\ Acceptation de paiement \\ Questionnaires modèles pour entretiens d'interception}

\section{Consentement en toute connaissance de cause}

Les premières tâches de l'enquêtrice consistent à se présenter, à informer la personne interrogée potentielle des objectifs, risques et avantages de la recherche et à obtenir le consentement de la personne à interroger. La plupart des organismes de recherche exigent que la personne interrogée donne son consentement en toute connaissance de cause, qui trouve son origine dans la recherche médicale, qui comprend des risques et des avantages. Les formulaires de consentement doivent utiliser des phrases simples et des déclarations directes, appropriées au niveau d'alphabétisation ou de scolarisation des personnes interrogées. Il faut donner au participant l'occasion de poser des questions et il doit être bien informé avant de signer le formulaire de consentement. Les principaux éléments d'un consentement en toute connaissance de cause sont les suivants :

1. But de l'étude.

2. Risques encourus par le participant à l'étude, y compris les risques physiques, sociaux et émotionnels.

3. Avantages que le participant peut tirer de l'étude, y compris, si nécessaire, une déclaration selon laquelle il n'en existe pas.

4. Informations relatives à la confidentialité, y compris l'utilisation d'identificateurs et l'accès à des données personnelles.

5. Où aller à tout moment en cas de question ou de problème.

6. Informations concernant le retrait de l'étude. Le participant a le droit de décider de ne plus en faire partie à quelque moment que ce soit, sans pénalisation.

7. Garantie que les services sont disponibles, que le participant potentiel décide ou non de prendre part à l'étude ou s'il décide de ne plus y participer.

8. Informations sur les procédures suivies et la durée de l'étude.

Le formulaire doit être sur une feuille de papier différente des autres. Il faut noter que l'enquêtrice doit pouvoir relier le formulaire de consentement au questionnaire approprié en leur donnant un numéro. Pour la collecte de données ne présentant qu'un risque peu élevé, un consentement oral suffit, mais le formulaire doit être signé par l'enquêtrice aux fins de documentation.

Chaque organisation a sa façon propre d'obtenir un consentement en toute connaissance de cause. Le questionnaire comporte un exemple de consentement en toute connaissance de cause. 


\section{Santé de la reproduction \\ Acceptation de paiement \\ Questionnaires modèles pour entretiens d'interception}

\section{MODELE POUR UTILISATEURS ACTUELS ET POTENTIELS DE SERVICES DE CLINIQUE}

[INSTRUCTIONS DESTINEE A L'ENQUETRICE : Il faut lire la déclaration suivante à toute personnes interrogée potentielle.] Puis-je vous demander un moment, s'il vous plaît? L'AGENCE désire continuer à vous offrir des services opportuns et de haute qualité. Pour cela, il est nécessaire qu'elle augmente les prix de certains services. Nous voudrions savoir comment ces changements de prix vous affecteront. Il n'y a pas de bonne ou de mauvaise réponse, alors soyez honnête et dites-nous ce qui est vrai pour vous. Les informations que nous collectons ne serviront qu'à des fins de planification et votre participation ne comporte ni risques, ni avantages personnels. Vous n'avez pas besoin de me donner votre nom et votre adresse. Tout ce que vous me direz restera confidentiel et le seul identificateur qui figure sur le questionnaire sera un numéro et non pas votre nom. Personne, y compris le personnel de ce dispensaire, ne saura ce que vous nous aurez dit personnellement et personne ne saura votre nom. Que vous acceptiez ou non de prendre part à cette étude, vous pourrez toujours avoir recours aux services. L'entretien prend environ quinze minutes. Vous pouvez l'abandonner n'importe quand sans conséquences. Avez-vous des questions? Voudriez-vous y participer? Si vous voulez en savoir davantage sur cette étude, veuillez contacter à

1. Participation OUI (effectuer l'entretien)

NON (remercier la personne et lui souhaiter une bonne journée)

[L'enquêtrice conservera tous les questionnaires, que la personne interrogée décide ou non de participer à l'enquête.]

2. Questionnaire numéro : [_] [_] [_] [ $]$

3. Dispensaire numéro : [__ $]$

4. Nom de l'enquêtrice :

5. Date de l'entretien : Jour Mois Année

6. Heure de début de l'entretien :

7. Heure de fin de l'entretien : 
A. Questions démographiques

\begin{tabular}{|c|c|c|c|}
\hline $\mathbf{Q}$. & QUESTION & CODE REPONSE & PASSER A \\
\hline 100 & $\begin{array}{l}\text { Quel âge aviez-vous lors de votre dernier } \\
\text { anniversaire? }\end{array}$ & Années & \\
\hline 101 & Quelle est votre situation de famille? & $\begin{array}{ll}\text { Mariée/Vivant avec quelqu'un } & 1 \\
\text { Veuve, séparée, divorcée } & 2 \\
\text { Célibataire } & 3 \\
\end{array}$ & \\
\hline 102 & Combien avez-vous d'enfants en vie ? & $\begin{array}{ll}\text { Aucun } & 00 \\
\text { Nombre } & \\
\end{array}$ & passer à 104 \\
\hline 103 & $\begin{array}{l}\text { Combien d'enfants en vie avez-vous dans les } \\
\text { groupes d'âges suivants? }\end{array}$ & \begin{tabular}{ll} 
GROUPE D'AGE & NO. D'ENFANTS \\
\cline { 2 - 2 } 1 & \\
1 à 3 & \\
4 ou plus & \\
\end{tabular} & \\
\hline 104 & $\begin{array}{l}\text { Combien d'années d'étude avez-vous faites? } \\
\text { DEMANDER QUELLE EST LA CLASSE LA } \\
\text { PLUS ELEVEE QUI A ETE TERMINEE }\end{array}$ & 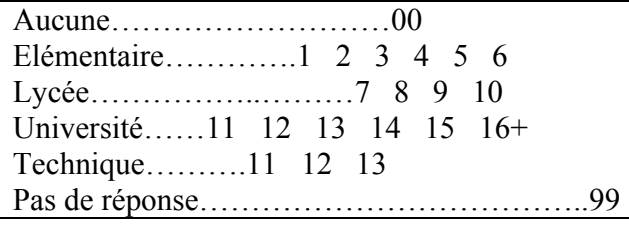 & \\
\hline
\end{tabular}

B Questions relatives à la capacité de payer

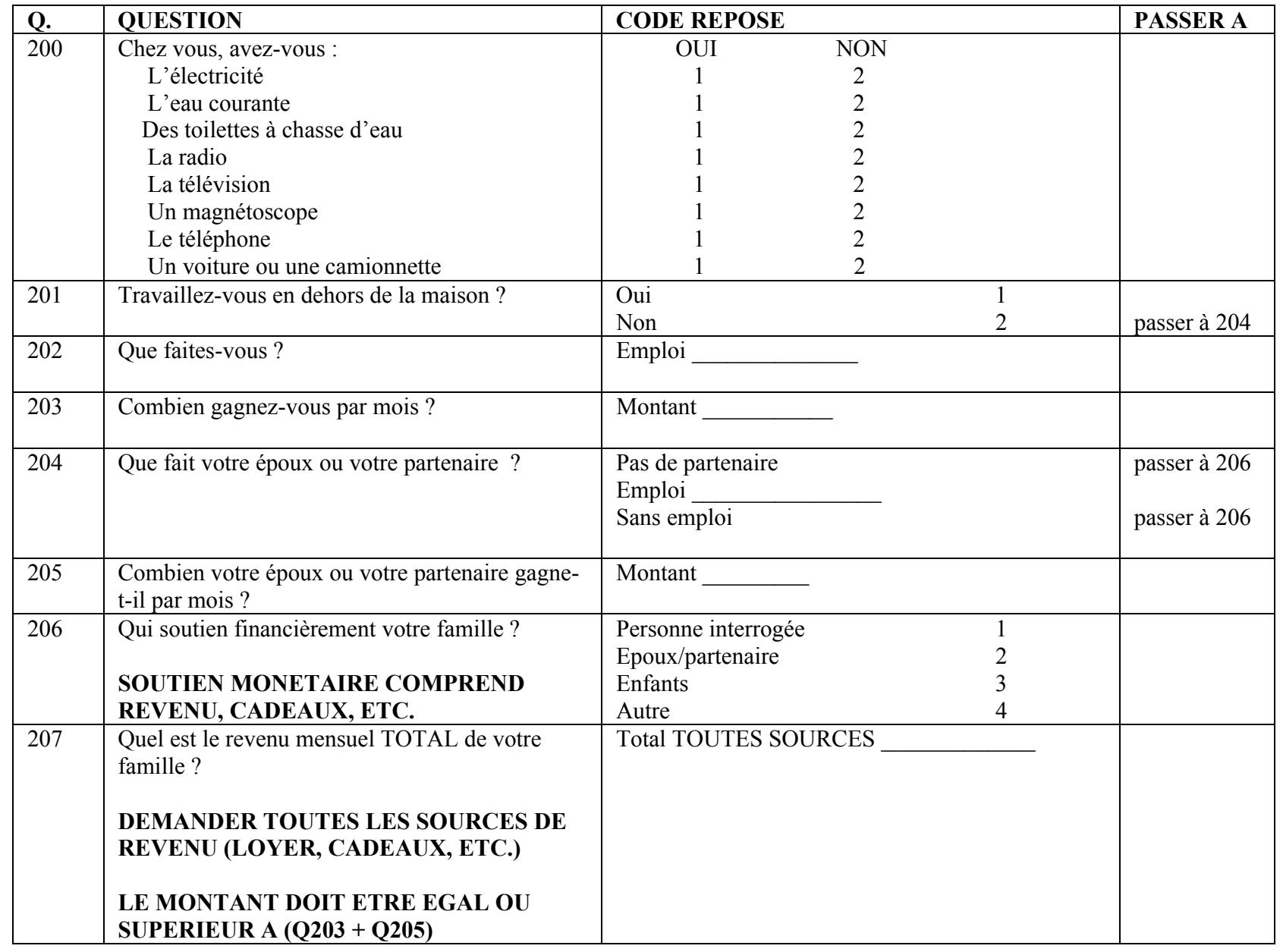


C. Type de consultation

\begin{tabular}{|c|c|c|c|c|}
\hline Q. & QUESTION & CODE REPONSE & & PASSER A \\
\hline 300 & Est-ce votre première visite à AGENCE ? & $\begin{array}{l}\text { Oui } \\
\text { Non }\end{array}$ & $\begin{array}{l}1 \\
2 \\
\end{array}$ & passer à 302 \\
\hline 301 & $\begin{array}{l}\text { Votre visite est-elle un « suivi » prévu lors } \\
\text { d'une consultation précédente? }\end{array}$ & $\begin{array}{l}\text { Oui } \\
\text { Non }\end{array}$ & $\begin{array}{l}1 \\
2 \\
\end{array}$ & \\
\hline 302 & $\begin{array}{l}\text { Quel est le principal service dont vous avez } \\
\text { bénéficié aujourd'hui? }\end{array}$ & $\begin{array}{l}\text { Gynécologie } \\
\text { Contraceptif injectable } \\
\text { Pose stérilet } \\
\text { Examen stérilet } \\
\text { Pilule } \\
\text { Soins prénatals } \\
\text { Autre : spécifier }\end{array}$ & $\begin{array}{l}1 \\
2 \\
3 \\
4 \\
5 \\
6 \\
\end{array}$ & $\begin{array}{l}\text { passer à } 400 \\
\text { passer à } 500 \\
\text { passer à } 600 \\
\text { passer à } 700 \\
\text { passer à } 800 \\
\text { passer à } 900 \\
\text { passer à FIN }\end{array}$ \\
\hline
\end{tabular}

\section{Questions relatives à l'acceptation de payer : utilisatrices actuelles}

\section{Gynécologie}

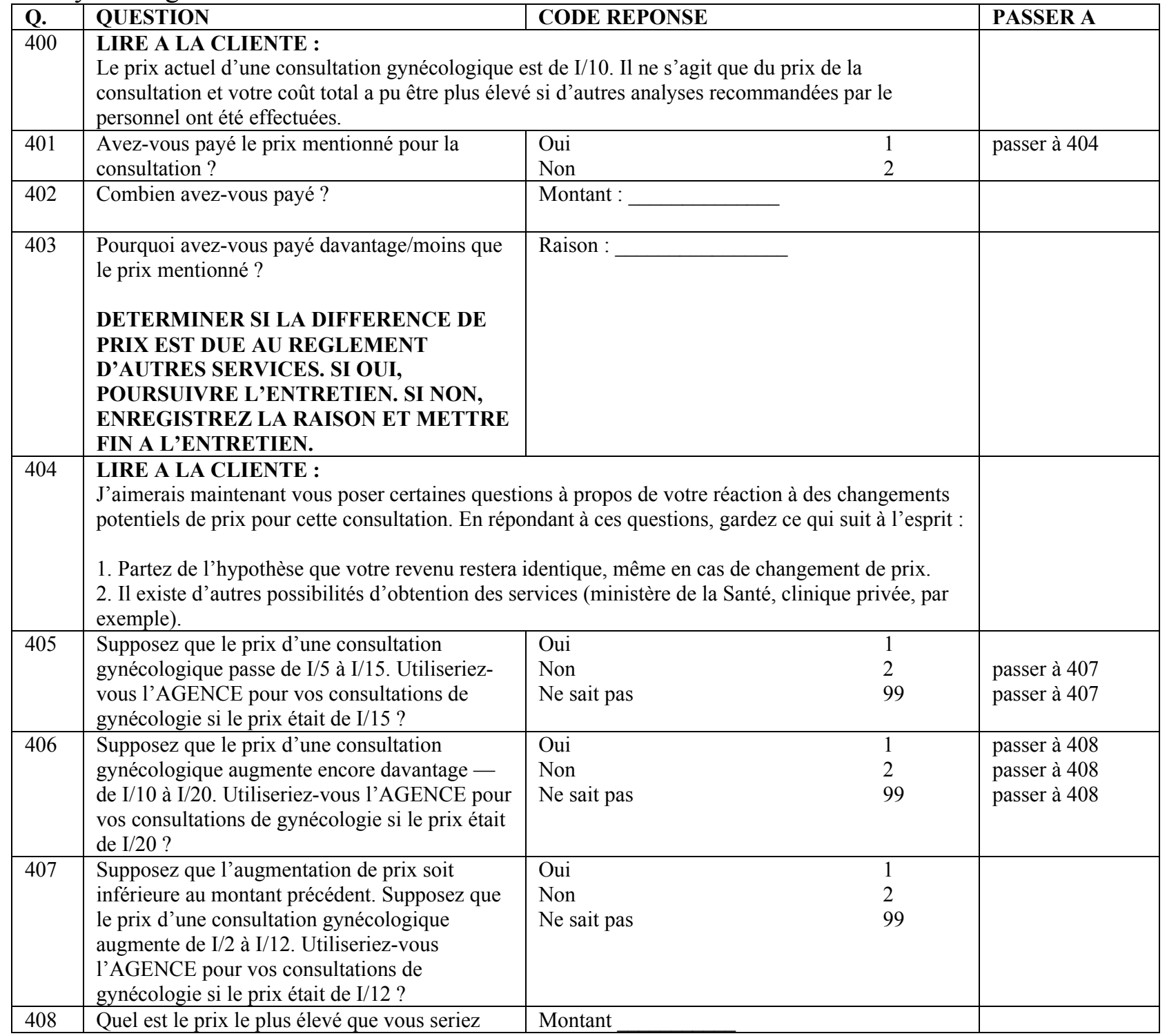




\begin{tabular}{|c|c|c|c|c|}
\hline & $\begin{array}{l}\text { prête à payer pour des services gynécologiques } \\
\text { de cette AGENCE? }\end{array}$ & & & \\
\hline 409 & $\begin{array}{l}\text { Si l'AGENCE augmentait le prix des services } \\
\text { de gynécologie au-delà que ce que vous prête à } \\
\text { payer ou capable de payer, que feriez-vous? }\end{array}$ & $\begin{array}{l}\text { S'en passer } \\
\text { Aller ailleurs } \\
\text { Ne sait pas } \\
\text { Autre : spécifier }\end{array}$ & $\begin{array}{l}1 \\
2 \\
99 \\
88\end{array}$ & $\begin{array}{l}\text { passer à } 1000 \\
\text { passer à } 410 \\
\text { passer à } 1000 \\
\text { passer à } 1000\end{array}$ \\
\hline 410 & $\begin{array}{l}\text { Où iriez-vous? } \\
\text { NE PAS DONNER LES CHOIX } \\
\text { DONNER LE CODE DE TOUS CEUX QUI } \\
\text { SONT MENTIONNES }\end{array}$ & $\begin{array}{l}\text { Secteur public } \\
\text { Autre ONG } \\
\text { Secteur privé } \\
\text { Pharmacie } \\
\text { Autre : spécifier } \\
\text { Je ne sais pas }\end{array}$ & $\begin{array}{l}1 \\
2 \\
3 \\
4 \\
88 \\
99\end{array}$ & $\begin{array}{l}\text { passer à } 1000 \\
\text { passer à } 1000 \\
\text { passer à } 1000 \\
\text { passer à } 1000 \\
\text { passer à } 1000 \\
\text { passer à } 1000\end{array}$ \\
\hline
\end{tabular}

\section{Contraceptif injectable}

\begin{tabular}{|c|c|c|c|c|}
\hline Q. & QUESTION & \multicolumn{2}{|l|}{ CODE REPONSE } & PASSER A \\
\hline 500 & \multicolumn{3}{|c|}{$\begin{array}{l}\text { LIRE A LA CLIENTE : } \\
\text { Le coût actuel du contraceptif injectable est de I/12. Il ne s'agit que du coût de la consultation et de } \\
\text { l'injection et votre coût total peut être plus élevé si d'autres analyses recommandées par le personnel } \\
\text { du dispensaire ont été effectuées. }\end{array}$} & \\
\hline 501 & Avez-vous payé le prix mentionné ? & $\begin{array}{l}\text { Oui } \\
\text { Non }\end{array}$ & $\begin{array}{l}1 \\
2\end{array}$ & passer à 504 \\
\hline 502 & Combien avez-vous payé ? & Montant : & & \\
\hline 503 & $\begin{array}{l}\text { Pourquoi avez-vous payé davantage/moins que } \\
\text { le prix mentionné? } \\
\text { DETERMINER SI LA DIFFERENCE DE } \\
\text { PRIX EST DUE AU REGLEMENT } \\
\text { D'AUTRES SERVICES. SI OUI, } \\
\text { POURSUIVRE L'ENTRETIEN. SI NON, } \\
\text { ENREGISTREZ LA RAISON ET METTRE } \\
\text { FIN A L'ENTRETIEN. }\end{array}$ & Raison & & \\
\hline 504 & \multicolumn{3}{|c|}{$\begin{array}{l}\text { LIRE A LA CLIENTE : } \\
\text { J'aimerais maintenant vous poser certaines questions à propos de votre réaction à des changements } \\
\text { potentiels de prix pour cette consultation. En répondant à ces questions, gardez ce qui suit à l'esprit : } \\
\text { 1. Partez de l'hypothèse que votre revenu restera identique, même en cas de changement de prix. } \\
\text { 2. Il existe d'autres possibilités d'obtention des services (ministère de la Santé, clinique privée, par } \\
\text { exemple). }\end{array}$} & \\
\hline 505 & $\begin{array}{l}\text { Supposez que le prix du contraceptif injectable } \\
\text { augmente de } \mathrm{I} / 5 \text { à } \mathrm{I} / 17 \text {. Viendriez-vous à } \\
\text { l'AGENCE pour votre injection si le prix était } \\
\text { de } \mathrm{I} / 17 \text { ? }\end{array}$ & $\begin{array}{l}\text { Oui } \\
\text { Non } \\
\text { Ne sait pas }\end{array}$ & $\begin{array}{l}1 \\
2 \\
99\end{array}$ & $\begin{array}{l}\text { passer à } 507 \\
\text { passer à } 507\end{array}$ \\
\hline 506 & $\begin{array}{l}\text { Supposez que le prix du contraceptif injectable } \\
\text { augmente encore davantage, de I/10 à I/22. } \\
\text { Viendriez-vous à l'AGENCE pour votre } \\
\text { injection si le prix était de } \mathrm{I} / 22 \text { ? }\end{array}$ & $\begin{array}{l}\text { Oui } \\
\text { Non } \\
\text { Ne sait pas }\end{array}$ & $\begin{array}{l}1 \\
2 \\
99\end{array}$ & $\begin{array}{l}\text { passer à } 508 \\
\text { passer à } 508 \\
\text { passer à } 508\end{array}$ \\
\hline 507 & $\begin{array}{l}\text { Supposez que l'augmentation de prix soit } \\
\text { inférieure au montant précédent. Supposez que } \\
\text { le prix du contraceptif injectable augmente de } \\
\mathrm{I} / 3 \text { à } \mathrm{I} / 15 \text {. Viendriez-vous à l'AGENCE pour } \\
\text { votre injection si le prix était de I/15? }\end{array}$ & $\begin{array}{l}\text { Oui } \\
\text { Non } \\
\text { Ne sait pas }\end{array}$ & $\begin{array}{l}1 \\
2 \\
99\end{array}$ & \\
\hline 508 & $\begin{array}{l}\text { Quel serait le prix le plus élevé que vous seriez } \\
\text { prête à payer pour le contraceptif injectable } \\
\text { dispensé par cette AGENCE? }\end{array}$ & Montant & & \\
\hline 509 & $\begin{array}{l}\text { Si l'AGENCE augmentait le prix du } \\
\text { contraceptif injectable au-delà de ce que vous } \\
\text { êtres prête à payer ou avez la capacité de payer, } \\
\text { que feriez-vous? }\end{array}$ & $\begin{array}{l}\text { N'utilise pas de moyen de contrac } \\
\text { Aller ailleurs } \\
\text { Passer à une méthode moins chère } \\
\quad \text { Spécifier : }\end{array}$ & $\begin{array}{l}\mathrm{n} \\
1 \\
2 \\
3\end{array}$ & $\begin{array}{l}\text { passer à } 1000 \\
\text { passer à } 510 \\
\text { passer à } 1000 \\
\text { passer à } 1000 \\
\text { passer à } 1000\end{array}$ \\
\hline
\end{tabular}




\begin{tabular}{|c|c|c|c|c|}
\hline & & $\begin{array}{l}\text { Autre : spécifier } \\
\text { Je ne sais pas }\end{array}$ & $\begin{array}{l}88 \\
99\end{array}$ & passer à 1000 \\
\hline 510 & $\begin{array}{l}\text { Où iriez-vous? } \\
\text { NE PAS DONNER LES CHOIX } \\
\text { DONNER LE CODE DE TOUS CEUX QUI } \\
\text { SONT MENTIONNES }\end{array}$ & $\begin{array}{l}\text { Secteur public } \\
\text { Autre ONG } \\
\text { Secteur privé } \\
\text { Pharmacie } \\
\text { Autre : spécifier } \\
\text { Je ne sais pas }\end{array}$ & $\begin{array}{l}1 \\
2 \\
3 \\
4 \\
88 \\
99 \\
\end{array}$ & $\begin{array}{l}\text { passer à } 1000 \\
\text { passer à } 1000 \\
\text { passer à } 1000 \\
\text { passer à } 1000 \\
\text { passer à } 1000 \\
\text { passer à } 1000\end{array}$ \\
\hline
\end{tabular}

3. Pose de stérilet

\begin{tabular}{|c|c|c|c|c|}
\hline Q. & QUESTION & \multicolumn{2}{|l|}{ CODE REPONSE } & PASSER A \\
\hline 600 & \multicolumn{3}{|c|}{$\begin{array}{l}\text { LIRE A LA CLIENTE : } \\
\text { Le prix actuel de la pose d'un stérilet est de I/15. Il ne s'agit que du coût de la consultation et du } \\
\text { stérilet, votre coût total peut être plus élevé si d'autres analyses recommandées par le personnel du } \\
\text { dispensaire ont été effectuées. }\end{array}$} & \\
\hline 601 & Avez-vous payé le prix mentionné ? & $\begin{array}{l}\text { Oui } \\
\text { Non }\end{array}$ & & passer à 604 \\
\hline 602 & Combien avez-vous payé ? & Montant: & & \\
\hline 603 & $\begin{array}{l}\text { Pourquoi avez-vous payé davantage/moins que } \\
\text { le prix mentionné? } \\
\text { DETERMINER SI LA DIFFERENCE DE } \\
\text { PRIX EST DUE AU REGLEMENT } \\
\text { D'AUTRES SERVICES. SI OUI, } \\
\text { POURSUIVRE L'ENTRETIEN. SI NON, } \\
\text { ENREGISTREZ LA RAISON ET METTRE } \\
\text { FIN A L'ENTRETIEN. }\end{array}$ & Raison : & & \\
\hline 604 & \multicolumn{3}{|c|}{$\begin{array}{l}\text { LIRE A LA CLIENTE : } \\
\text { J'aimerais maintenant vous poser certaines questions à propos de votre réaction à des changements } \\
\text { potentiels de prix pour cette consultation. En répondant à ces questions, gardez ce qui suit à l'esprit : } \\
\text { 1. Partez de l'hypothèse que votre revenu restera identique, même en cas de changement de prix. } \\
\text { 2. Il existe d'autres possibilités d'obtention des services (ministère de la Santé, clinique privée, par } \\
\text { exemple). }\end{array}$} & \\
\hline 605 & $\begin{array}{l}\text { Supposez que le prix de la pose d'un stérilet } \\
\text { augmente de } I / 5 \text { à } I / 20 \text {, viendriez-vous à } \\
\text { l'AGENCE pour la pose d'un stérilet si le prix } \\
\text { était de } I / 20 \text { ? }\end{array}$ & $\begin{array}{l}\text { Oui } \\
\text { Non } \\
\text { Ne sait pas }\end{array}$ & $\begin{array}{l}1 \\
2 \\
99\end{array}$ & $\begin{array}{l}\text { passer à } 607 \\
\text { passer à } 607\end{array}$ \\
\hline 606 & $\begin{array}{l}\text { Supposez que le prix de la pose d'un stérilet } \\
\text { augmente encore davantage, de I/10 à I/25. } \\
\text { Viendriez-vous à l'AGENCE pour la pose d'un } \\
\text { stérilet si le prix était de } \mathrm{I} / 25 \text { ? }\end{array}$ & $\begin{array}{l}\text { Oui } \\
\text { Non } \\
\text { Ne sait pas }\end{array}$ & $\begin{array}{l}1 \\
2 \\
99\end{array}$ & $\begin{array}{l}\text { passer à } 608 \\
\text { passer à } 608 \\
\text { passer à } 608\end{array}$ \\
\hline 607 & $\begin{array}{l}\text { Supposez que l'augmentation de prix soit } \\
\text { inférieure au montant précédent. Supposez que } \\
\text { le prix de la pose d'un stérilet augmente de I/3 à } \\
\text { I/18. Viendriez-vous à l'AGENCE pour la pose } \\
\text { d'un stérilet si le prix était de I/18? }\end{array}$ & $\begin{array}{l}\text { Oui } \\
\text { Non } \\
\text { Ne sait pas }\end{array}$ & $\begin{array}{l}1 \\
2 \\
99\end{array}$ & \\
\hline 608 & $\begin{array}{l}\text { Quel serait le prix le plus élevé que vous seriez } \\
\text { prête à payer pour la pose d'un stérilet dans } \\
\text { cette AGENCE? }\end{array}$ & Montant & & \\
\hline 609 & $\begin{array}{l}\text { Si l'AGENCE augmentait le prix de la pose } \\
\text { d'un stérilet au-delà de ce que vous êtes prête à } \\
\text { payer ou de votre capacité à payer, que feriez- } \\
\text { vous? }\end{array}$ & $\begin{array}{l}\text { N'utilise pas de moyen de con } \\
\text { Aller ailleurs } \\
\text { Passer à un moyen moins cher } \\
\quad \text { Spécifier: } \\
\text { Autre : spécifier } \\
\text { Je ne sais pas }\end{array}$ & $\begin{array}{l}0 \\
1 \\
2 \\
3 \\
88 \\
99\end{array}$ & $\begin{array}{l}\text { passer à } 1000 \\
\text { passer à } 610 \\
\text { passer à } 1000 \\
\text { passer à } 1000 \\
\text { passer à } 1000 \\
\text { passer à } 1000\end{array}$ \\
\hline 610 & Où iriez-vous? & $\begin{array}{l}\text { Secteur public } \\
\text { Autre ONG } \\
\text { Secteur privé }\end{array}$ & $\begin{array}{l}1 \\
2 \\
3\end{array}$ & $\begin{array}{l}\text { passer à } 1000 \\
\text { passer à } 1000 \\
\text { passer à } 1000\end{array}$ \\
\hline
\end{tabular}




\begin{tabular}{|l|l|ll|l|}
\hline & NE PAS DONNER LES CHOIX & Pharmacie & 4 & passer à 1000 \\
& DONNER LE CODE DE TOUS CEUX QUI & Autre : spécifier & 88 & passer à 1000 \\
& SONT MENTIONNES & Je ne sais pas & 99 & passer à 1000 \\
\hline
\end{tabular}

\section{Examen stérilet}

\begin{tabular}{|c|c|c|c|c|}
\hline Q. & QUESTION & \multicolumn{2}{|l|}{ CODE REPONSE } & PASSER A \\
\hline 700 & \multicolumn{3}{|c|}{$\begin{array}{l}\text { LIRE A LA CLIENTE : } \\
\text { Le coût actuel d'un examen stérilet est de I/10. Il ne s'agit que du prix de la consultation, votre coût } \\
\text { total peut être plus élevé si d'autres analyses recommandées par le personnel du dispensaire ont été } \\
\text { effectuées. }\end{array}$} & \\
\hline 701 & Avez-vous payé le prix mentionné ? & $\begin{array}{l}\text { Oui } \\
\text { Non }\end{array}$ & & passer à 704 \\
\hline 702 & Combien avez-vous payé ? & Montant & & \\
\hline 703 & $\begin{array}{l}\text { Pourquoi avez-vous payé davantage/moins que } \\
\text { le prix mentionné? } \\
\text { DETERMINER SI LA DIFFERENCE DE } \\
\text { PRIX EST DUE AU REGLEMENT } \\
\text { D'AUTRES SERVICES. SI OUI, } \\
\text { POURSUIVRE L'ENTRETIEN. SI NON, } \\
\text { ENREGISTREZ LA RAISON ET METTRE } \\
\text { FIN A L'ENTRETIEN. }\end{array}$ & Raison: & & \\
\hline 704 & \multicolumn{3}{|c|}{$\begin{array}{l}\text { LIRE A LA CLIENTE : } \\
\text { J'aimerais maintenant vous poser certaines questions à propos de votre réaction à des changements } \\
\text { potentiels de prix pour cette consultation. En répondant à ces questions, gardez ce qui suit à l'esprit : } \\
\text { 1. Partez de l'hypothèse que votre revenu restera identique, même en cas de changement de prix. } \\
\text { 2. Il existe d'autres possibilités d'obtention des services (ministère de la Santé, clinique privée, par } \\
\text { exemple). }\end{array}$} & \\
\hline 705 & $\begin{array}{l}\text { Supposez que le prix d'un examen stérilet } \\
\text { augmente de I/5 à I/15. Viendriez-vous à } \\
\text { l'AGENCE pour votre examen stérilet su le } \\
\text { prix était de I/15? }\end{array}$ & $\begin{array}{l}\text { Oui } \\
\text { Non } \\
\text { Ne sait pas }\end{array}$ & $\begin{array}{l}1 \\
2 \\
99\end{array}$ & $\begin{array}{l}\text { passer à } 707 \\
\text { passer à } 707\end{array}$ \\
\hline 706 & $\begin{array}{l}\text { Supposez que le prix d'un examen stérilet } \\
\text { augmente encore davantage, de } \mathrm{I} / 10 \text { à } \mathrm{I} / 20 \text {. } \\
\text { Viendriez-vous à l'AGENCE pour votre } \\
\text { examen stérilet su le prix était de } \mathrm{I} / 20 \text { ? }\end{array}$ & $\begin{array}{l}\text { Oui } \\
\text { Non } \\
\text { Ne sait pas }\end{array}$ & $\begin{array}{l}1 \\
2 \\
99\end{array}$ & $\begin{array}{l}\text { passer à } 708 \\
\text { passer à } 708 \\
\text { passer à } 708\end{array}$ \\
\hline 707 & $\begin{array}{l}\text { Supposez que l'augmentation de prix soit } \\
\text { inférieure au montant précédent. Supposez que } \\
\text { le prix d'un examen stérilet augmente de I/2 à } \\
\text { I/12. Viendriez-vous à l'AGENCE pour votre } \\
\text { examen stérilet su le prix était de I/12? }\end{array}$ & $\begin{array}{l}\text { Oui } \\
\text { Non } \\
\text { Ne sait pas }\end{array}$ & $\begin{array}{l}1 \\
2 \\
99\end{array}$ & \\
\hline 708 & $\begin{array}{l}\text { Quel est le prix le plus élevé que vous seriez } \\
\text { prête à payer pour un examen stérilet par } \\
\text { l'AGENCE? }\end{array}$ & Montant & & \\
\hline 709 & $\begin{array}{l}\text { Si l'AGENCE augmentait le prix d'un examen } \\
\text { stérilet au-delà de ce que vous êtes prête à payer } \\
\text { ou avez la capacité de payer, que feriez-vous? }\end{array}$ & $\begin{array}{l}\text { S'en passer } \\
\text { Aller ailleurs } \\
\text { Je ne sais pas } \\
\text { Autre : spécifier }\end{array}$ & $\begin{array}{l}1 \\
2 \\
99 \\
88\end{array}$ & $\begin{array}{l}\text { passer à } 1000 \\
\text { passer à } 710 \\
\text { passer à } 1000 \\
\text { passer à } 1000\end{array}$ \\
\hline 710 & $\begin{array}{l}\text { Où iriez-vous? } \\
\text { NE PAS DONNER LES CHOIX } \\
\text { DONNER LE CODE DE TOUS CEUX QUI } \\
\text { SONT MENTIONNES }\end{array}$ & $\begin{array}{l}\text { Secteur public } \\
\text { Autre ONG } \\
\text { Secteur privé } \\
\text { Pharmacie } \\
\text { Autre : spécifier } \\
\text { Je ne sais pas }\end{array}$ & $\begin{array}{l}1 \\
2 \\
3 \\
4 \\
88 \\
99\end{array}$ & $\begin{array}{l}\text { passer à } 1000 \\
\text { passer à } 1000 \\
\text { passer à } 1000 \\
\text { passer à } 1000 \\
\text { passer à } 1000 \\
\text { passer à } 1000\end{array}$ \\
\hline
\end{tabular}


5. Achat de pilule

\begin{tabular}{|c|c|c|c|c|}
\hline Q. & QUESTION & \multicolumn{2}{|l|}{ CODE REPONSE } & PASSER A \\
\hline 800 & \multicolumn{3}{|c|}{$\begin{array}{l}\text { LIRE A LA CLIENTE : } \\
\text { Le prix actuel du contraceptif par voie orale MARQUE est de I/3. Il ne s'agit que du prix des pilules } \\
\text { pour un cycle, votre coût total peut être plus élevé si d'autres analyses recommandées par le } \\
\text { personnel du dispensaire ont été effectuées. }\end{array}$} & \\
\hline 801 & Avez-vous payé le prix mentionné ? & $\begin{array}{l}\text { Oui } \\
\text { Non }\end{array}$ & $\begin{array}{l}1 \\
2\end{array}$ & passer à 804 \\
\hline 802 & Combien avez-vous payé ? & Montant: & & \\
\hline 803 & $\begin{array}{l}\text { Pourquoi avez-vous payé davantage/moins que } \\
\text { le prix mentionné? } \\
\text { DETERMINER SI LA DIFFERENCE DE } \\
\text { PRIX EST DUE AU REGLEMENT } \\
\text { D'AUTRES SERVICES. SI OUI, } \\
\text { POURSUIVRE L'ENTRETIEN. SI NON, } \\
\text { ENREGISTREZ LA RAISON ET METTRE } \\
\text { FIN A L'ENTRETIEN. }\end{array}$ & Raison : & & \\
\hline 804 & \multicolumn{3}{|c|}{$\begin{array}{l}\text { LIRE A LA CLIENTE : } \\
\text { J'aimerais maintenant vous poser certaines questions à propos de votre réaction à des changements } \\
\text { potentiels de prix pour cette consultation. En répondant à ces questions, gardez ce qui suit à l'esprit : } \\
\text { 1. Partez de l'hypothèse que votre revenu restera identique, même en cas de changement de prix. } \\
\text { 2. Il existe d'autres possibilités d'obtention des services (ministère de la Santé, clinique privée, par } \\
\text { exemple). }\end{array}$} & \\
\hline 805 & $\begin{array}{l}\text { Supposez que le prix des contraceptifs par voie } \\
\text { orale MARQUE augmente de } \mathrm{I} / 2 \text { à } \mathrm{I} / 5 \text {. } \\
\text { Achèteriez-vous vos pilules à l'AGENCE si le } \\
\text { prix était de } \mathrm{I} / 5 \text { ? }\end{array}$ & $\begin{array}{l}\text { Oui } \\
\text { Non } \\
\text { Ne sait pas }\end{array}$ & $\begin{array}{l}1 \\
2 \\
99\end{array}$ & $\begin{array}{l}\text { passer à } 807 \\
\text { passer à } 807\end{array}$ \\
\hline 806 & $\begin{array}{l}\text { Supposez que le prix des contraceptifs par voie } \\
\text { orale MARQUE augmente encore davantage, } \\
\text { de I/3 à } I / 6 \text {. Achèteriez-vous vos pilules à } \\
\text { l'AGENCE si le prix était de } I / 6 \text { ? }\end{array}$ & $\begin{array}{l}\text { Oui } \\
\text { No } \\
\text { Ne sait pas }\end{array}$ & $\begin{array}{l}1 \\
2 \\
99\end{array}$ & $\begin{array}{l}\text { passer à } 808 \\
\text { passer à } 808 \\
\text { passer à } 808\end{array}$ \\
\hline 807 & $\begin{array}{l}\text { Supposez que l'augmentation du prix soit } \\
\text { inférieure au montant précédent. Supposez que } \\
\text { le prix des contraceptifs par voie orale } \\
\text { MARQUE augmente de I/1 à I/4. Achèteriez- } \\
\text { vous vos pilules à l'AGENCE si le prix était de } \\
\text { I/4? }\end{array}$ & $\begin{array}{l}\text { Oui } \\
\text { Non } \\
\text { Ne sait pas }\end{array}$ & $\begin{array}{l}1 \\
2 \\
99\end{array}$ & \\
\hline 808 & $\begin{array}{l}\text { Quel serait le prix le plus élevé que vous seriez } \\
\text { prête à payer pour les contraceptifs par voie } \\
\text { orale MARQUE achetés à cette AGENCE? }\end{array}$ & Montant & & \\
\hline 809 & $\begin{array}{l}\text { Si l'AGENCE augmentait le prix des } \\
\text { contraceptifs par voie orale MARQUE au-delà } \\
\text { de ce que êtes prête à payer ou avez la capacité } \\
\text { de payer, que feriez-vous? }\end{array}$ & $\begin{array}{l}\text { N'utilise pas de moyen de cont } \\
\text { Aller ailleurs } \\
\text { Passer à un moyen moins cher } \\
\quad \text { Spécifier : } \\
\text { Autre : spécifier } \\
\text { Je ne sais pas }\end{array}$ & $\begin{array}{l}1 \\
2 \\
3 \\
88 \\
99\end{array}$ & $\begin{array}{l}\text { passer à } 1000 \\
\text { passer à } 810 \\
\text { passer à } 1000 \\
\text { passer à } 1000 \\
\text { passer à } 1000 \\
\text { passer à } 1000\end{array}$ \\
\hline 810 & $\begin{array}{l}\text { Où iriez-vous? } \\
\text { NE PAS DONNER LES CHOIX } \\
\text { DONNER LE CODE DE TOUS CEUX QUI } \\
\text { SONT MENTIONNES }\end{array}$ & $\begin{array}{l}\text { Secteur public } \\
\text { Autre ONG } \\
\text { Secteur privé } \\
\text { Pharmacie } \\
\text { Autre : spécifier } \\
\text { Je ne sais pas }\end{array}$ & $\begin{array}{l}1 \\
2 \\
3 \\
4 \\
88 \\
99\end{array}$ & $\begin{array}{l}\text { passer à } 1000 \\
\text { passer à } 1000 \\
\text { passer à } 1000 \\
\text { passer à } 1000 \\
\text { passer à } 1000 \\
\text { passer à } 1000\end{array}$ \\
\hline
\end{tabular}


6. Soins prénatals

\begin{tabular}{|c|c|c|c|c|}
\hline Q. & QUESTION & \multicolumn{2}{|c|}{ CODE REPONSE } & PASSER A \\
\hline 900 & \multicolumn{3}{|c|}{$\begin{array}{l}\text { READ TO CLIENT: } \\
\text { Le coût actuel d'une consultation pour soins prénatals est de I/ } 15 \text {. Il ne s'agit que du prix de la } \\
\text { consultation et votre coût total a pu être plus élevé si d'autres analyses recommandées par le } \\
\text { personnel ont été effectuées. }\end{array}$} & \\
\hline 901 & Avez-vous payé le prix mentionné ? & $\begin{array}{l}\text { Oui } \\
\text { Non }\end{array}$ & $\begin{array}{l}1 \\
2\end{array}$ & passer à 904 \\
\hline 902 & Combien avez-vous payé ? & Montant : & & \\
\hline 903 & $\begin{array}{l}\text { Pourquoi avez-vous payé davantage/moins que } \\
\text { le prix mentionné ? } \\
\text { DETERMINER SI LA DIFFERENCE DE } \\
\text { PRIX EST DUE AU REGLEMENT } \\
\text { D'AUTRES SERVICES. SI OUI, } \\
\text { POURSUIVRE L'ENTRETIEN. SI NON, } \\
\text { ENREGISTREZ LA RAISON ET METTRE } \\
\text { FIN A L'ENTRETIEN. }\end{array}$ & Raison : & & \\
\hline 904 & \multicolumn{3}{|c|}{$\begin{array}{l}\text { LIRE A LA CLIENTE : } \\
\text { J'aimerais maintenant vous poser certaines questions à propos de votre réaction à des changements } \\
\text { potentiels de prix pour cette consultation. En répondant à ces questions, gardez ce qui suit à l'esprit } \\
\text { 1. Partez de l'hypothèse que votre revenu restera identique, même en cas de changement de prix. } \\
\text { 2. Il existe d'autres possibilités d'obtention des services (ministère de la Santé, clinique privée, par } \\
\text { exemple). }\end{array}$} & \\
\hline 905 & $\begin{array}{l}\text { Supposez que le prix des soins prénatals } \\
\text { augmente de } \mathrm{I} / 5 \text { à } \mathrm{I} / 20 \text {. Viendriez-vous à } \\
\text { l'AGENCE si le prix des soins prénatals était } \\
\text { de } \mathrm{I} / 20 \text { ? }\end{array}$ & $\begin{array}{l}\text { Oui } \\
\text { Non } \\
\text { Ne sait pas }\end{array}$ & $\begin{array}{l}1 \\
2 \\
99\end{array}$ & $\begin{array}{l}\text { passer à } 907 \\
\text { passer à } 907\end{array}$ \\
\hline 906 & $\begin{array}{l}\text { Supposez que le prix des soins prénatals } \\
\text { augmente encore davantage, de } \mathrm{I} / 10 \text { à } \mathrm{I} / 25 \text {. } \\
\text { Viendriez-vous à l'AGENCE si le prix des } \\
\text { soins prénatals était de } \mathrm{I} / 25 \text { ? }\end{array}$ & $\begin{array}{l}\text { Oui } \\
\text { Non } \\
\text { Ne sait pas }\end{array}$ & $\begin{array}{l}1 \\
2 \\
99\end{array}$ & $\begin{array}{l}\text { PASSER À } 908 \\
\text { passer à } 908 \\
\text { passer à } 908\end{array}$ \\
\hline 907 & $\begin{array}{l}\text { Supposez que l'augmentation de prix soit } \\
\text { inférieure au montant précédent. Supposez que } \\
\text { le prix des soins prénatals augmente de I/2 à } \\
\text { I/17. Viendriez-vous à l'AGENCE si le prix des } \\
\text { soins prénatals était de I/17? }\end{array}$ & $\begin{array}{l}\text { Oui } \\
\text { Non } \\
\text { Ne sait pas }\end{array}$ & $\begin{array}{l}1 \\
2 \\
99\end{array}$ & \\
\hline 908 & $\begin{array}{l}\text { Quel serait le prix le plus élevé que vous seriez } \\
\text { prête à payer pour des soins prénatals dispensés } \\
\text { par l'AGENCE? }\end{array}$ & Montant & & \\
\hline 909 & $\begin{array}{l}\text { Si l'AGENCE augmentait le prix des soins } \\
\text { prénatals au-delà de ce que vous êtes prête à } \\
\text { payer ou avez la capacité de payer, que feriez- } \\
\text { vous? }\end{array}$ & $\begin{array}{l}\text { S'en passer } \\
\text { Aller ailleurs } \\
\text { Je ne sais pas } \\
\text { Autre : spécifier }\end{array}$ & $\begin{array}{l}1 \\
2 \\
99 \\
88\end{array}$ & $\begin{array}{l}\text { passer à } 1000 \\
\text { passer à } 910 \\
\text { passer à } 1000 \\
\text { passer à } 1000\end{array}$ \\
\hline 910 & $\begin{array}{l}\text { Où iriez-vous? } \\
\text { NE PAS DONNER LES CHOIX } \\
\text { DONNER LE CODE DE TOUS CEUX QUI } \\
\text { SONT MENTIONNES }\end{array}$ & $\begin{array}{l}\text { Secteur public } \\
\text { Autre ONG } \\
\text { Secteur privé } \\
\text { Pharmacie } \\
\text { Autre : spécifier } \\
\text { Je ne sais pas }\end{array}$ & $\begin{array}{l}1 \\
2 \\
3 \\
4 \\
88 \\
99\end{array}$ & $\begin{array}{l}\text { passer à } 1000 \\
\text { passer à } 1000 \\
\text { passer à } 1000 \\
\text { passer à } 1000 \\
\text { passer à } 1000 \\
\text { passer à } 1000\end{array}$ \\
\hline
\end{tabular}




\section{(LES QUESTIONS SUIVANTES SONT POSEES A TOUTES LES PERSONNES INTERROGEES QUI ONT FINI UNE SEQUENCE WTP PRECEDENTE)}

\section{E. Questions WTP : Utilisateurs potentiels}

1. Stérilisation féminine

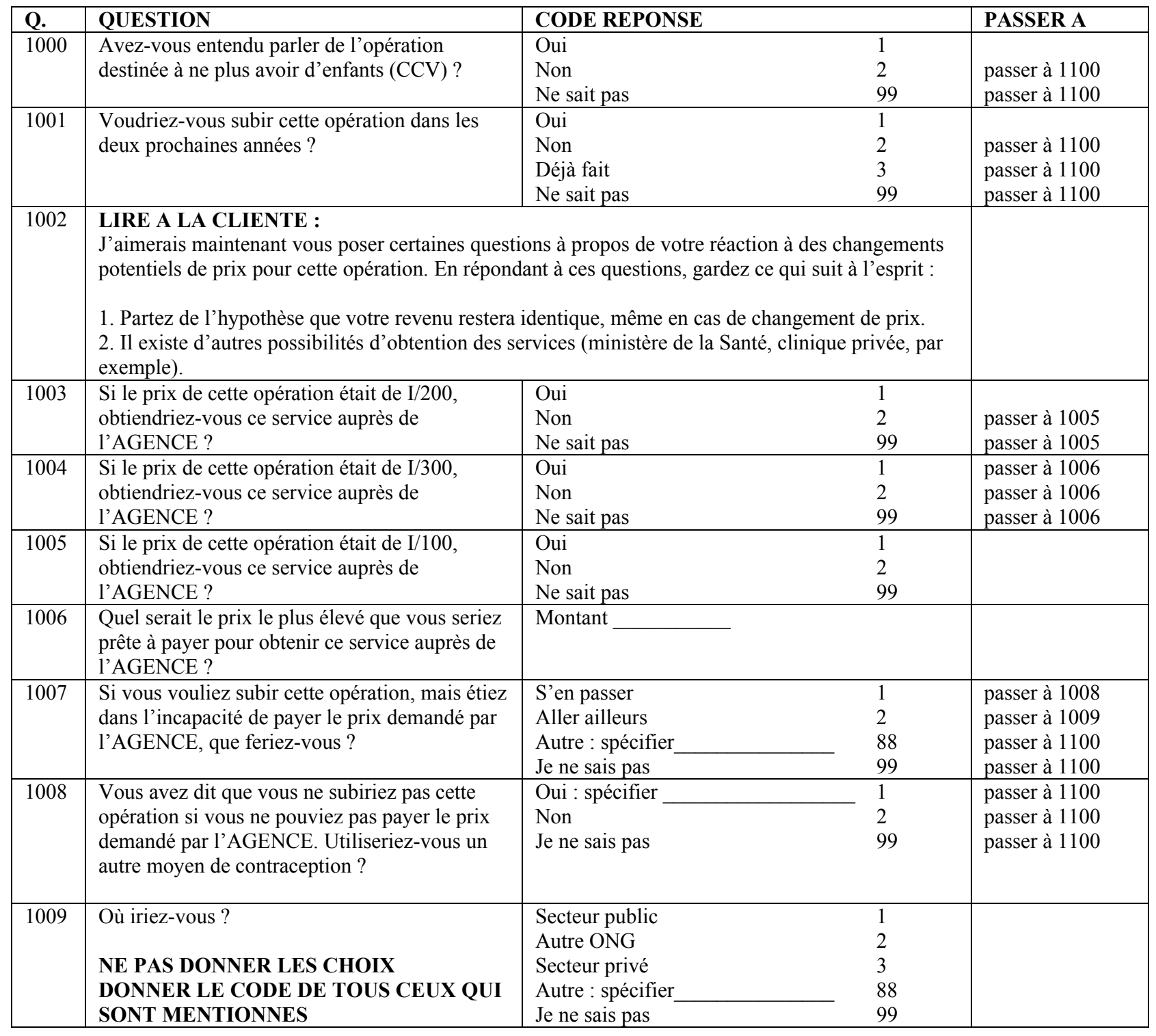

\section{F. Acceptation de paiement pour l'amélioration des accommodements et de la qualité}

1. Acceptation de paiement pour la réduction du temps d'attente

\begin{tabular}{|c|c|c|c|c|}
\hline $\mathbf{Q}$. & QUESTION & CODE REPONSE & & PASSER A \\
\hline 1100 & $\begin{array}{l}\text { Combien de temps avez-vous dû attendre avant } \\
\text { de voir un membre du personnel de ce } \\
\text { dispensaire? }\end{array}$ & Temps en minutes & & \\
\hline 1101 & $\begin{array}{l}\text { Considérez-vous que ce temps d'attente est } \\
\text { excessif, raisonnable ou court? }\end{array}$ & $\begin{array}{l}\text { Excessif } \\
\text { Raisonnable } \\
\text { Court }\end{array}$ & $\begin{array}{l}1 \\
2 \\
3\end{array}$ & \\
\hline 1102 & Supposez que le temps d'attente soit réduit de & Oui & 1 & \\
\hline
\end{tabular}




\begin{tabular}{|c|c|c|c|c|}
\hline & $\begin{array}{l}\text { moitié, mais que le prix de la consultation soit } \\
\text { augmenté de } 3 \text {, c'est-à-dire MONTANT } \\
\text { PAYE }+3 \text {. Seriez-vous prêt à payer pour cette } \\
\text { amélioration? }\end{array}$ & $\begin{array}{l}\text { Non } \\
\text { Ne sait pas }\end{array}$ & $\begin{array}{c}2 \\
99\end{array}$ & $\begin{array}{l}\text { passer à } 1104 \\
\text { passer à } 1104\end{array}$ \\
\hline 1103 & $\begin{array}{l}\text { Supposez que le temps d'attendre soit réduit de } \\
\text { moitié mais que le prix de la consultation soit } \\
\text { augmenté de } 6 \text {, soit MONTANT PAYE + 6. } \\
\text { Seriez-vous prêt à payer pour cette } \\
\text { amélioration? }\end{array}$ & $\begin{array}{l}\text { Oui } \\
\text { Non } \\
\text { Ne sait pas }\end{array}$ & $\begin{array}{c}1 \\
2 \\
99\end{array}$ & $\begin{array}{l}\text { passer à } 1105 \\
\text { passer à } 1105 \\
\text { passer à } 1105\end{array}$ \\
\hline 1104 & $\begin{array}{l}\text { Supposez que le temps d'attente soit réduit de } \\
\text { moitié, mais que le prix de la consultation soit } \\
\text { augmenté de 1, soit MONTANT PAYE + 1. } \\
\text { Seriez-vous prêt à payer pour cette amélioration } \\
\text { ? }\end{array}$ & $\begin{array}{l}\text { Oui } \\
\text { Non } \\
\text { Ne sait pas }\end{array}$ & $\begin{array}{c}1 \\
2 \\
99\end{array}$ & \\
\hline 1105 & $\begin{array}{l}\text { Quel est le prix le plus élevé que vous seriez } \\
\text { prêt à payer pour votre consultation si cette } \\
\text { amélioration était apportée? }\end{array}$ & Montant & & \\
\hline
\end{tabular}

2. Acceptation de paiement pour counseling

\begin{tabular}{|c|c|c|c|c|}
\hline Q. & QUESTION & CODE REPONSE & & PASSER A \\
\hline 1200 & $\begin{array}{l}\text { Combien de temps le prestataire de service a-t- } \\
\text { il passé à vous expliquer les procédures et à } \\
\text { vous conseiller à propos de vos problèmes de } \\
\text { santé de la reproduction? }\end{array}$ & Temps en minutes & & \\
\hline 1201 & $\begin{array}{l}\text { Considérez-vous que le temps que votre } \\
\text { conseiller a passé avec vous est excessif, } \\
\text { raisonnable ou court? }\end{array}$ & $\begin{array}{l}\text { Excessif } \\
\text { Raisonnable } \\
\text { Court }\end{array}$ & $\begin{array}{l}1 \\
2 \\
3\end{array}$ & passer à 1300 \\
\hline 1202 & $\begin{array}{l}\text { Supposez que l'on augmente de } 10 \text { minutes le } \\
\text { temps que vous pouvez passer avec votre } \\
\text { conseiller, mais que le prix de la consultation } \\
\text { augmente de 3, soit MONTANT PAYE }+\mathbf{3} \text {. } \\
\text { Seriez-vous prêt à payer pour cette } \\
\text { amélioration? }\end{array}$ & $\begin{array}{l}\text { Oui } \\
\text { Non } \\
\text { Ne sait pas }\end{array}$ & $\begin{array}{c}1 \\
2 \\
99\end{array}$ & $\begin{array}{l}\text { passer à } 1204 \\
\text { passer à } 1204\end{array}$ \\
\hline 1203 & $\begin{array}{l}\text { Supposez que l'on augmente de } 10 \text { minutes le } \\
\text { temps que vous pouvez passer avec votre } \\
\text { conseiller, mais que le prix de la consultation } \\
\text { augmente de } 6 \text {, soit MONTANT PAYE + 6. } \\
\text { Seriez-vous prêt à payer pour cette } \\
\text { amélioration? }\end{array}$ & $\begin{array}{l}\text { Oui } \\
\text { Non } \\
\text { Ne sait pas }\end{array}$ & $\begin{array}{c}1 \\
2 \\
99\end{array}$ & $\begin{array}{l}\text { passer à } 1205 \\
\text { passer à } 1205 \\
\text { passer à } 1205\end{array}$ \\
\hline 1204 & $\begin{array}{l}\text { Supposez que l'on augmente de } 10 \text { minutes le } \\
\text { temps que vous pouvez passer avec votre } \\
\text { conseiller, mais que le prix de la consultation } \\
\text { augmente de } 1 \text {, soit MONTANT PAYE }+\mathbf{1} \text {. } \\
\text { Seriez-vous prêt à payer pour cette } \\
\text { amélioration? }\end{array}$ & $\begin{array}{l}\text { Oui } \\
\text { Non } \\
\text { Ne sait pas }\end{array}$ & $\begin{array}{c}1 \\
2 \\
99\end{array}$ & \\
\hline 1205 & $\begin{array}{l}\text { Quel est le prix le plus élevé que vous seriez } \\
\text { prêt à payer si cette amélioration était } \\
\text { apportée? }\end{array}$ & Montant & & \\
\hline
\end{tabular}

3. Acceptation de paiement pour l'amélioration du dispensaire

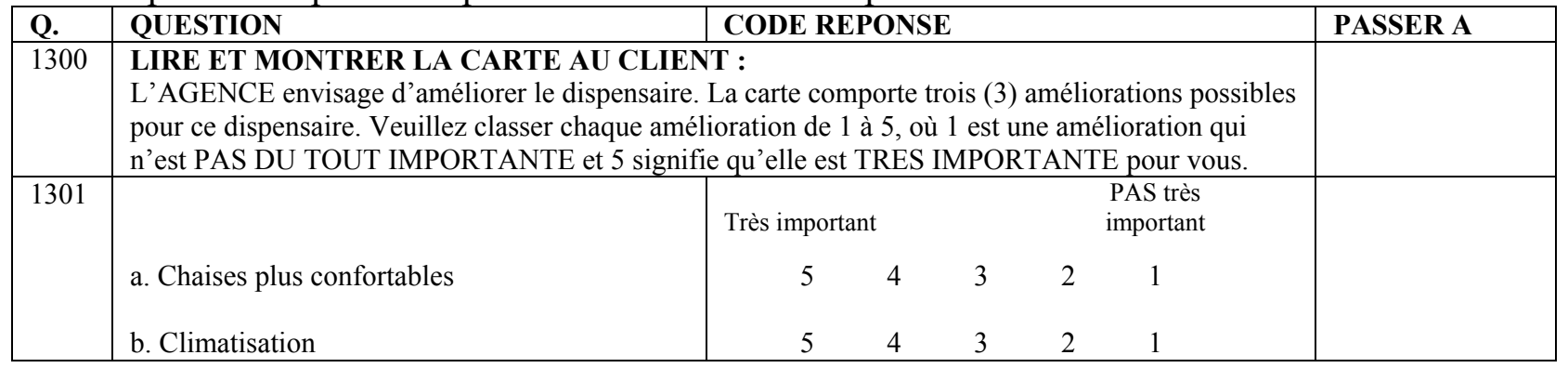




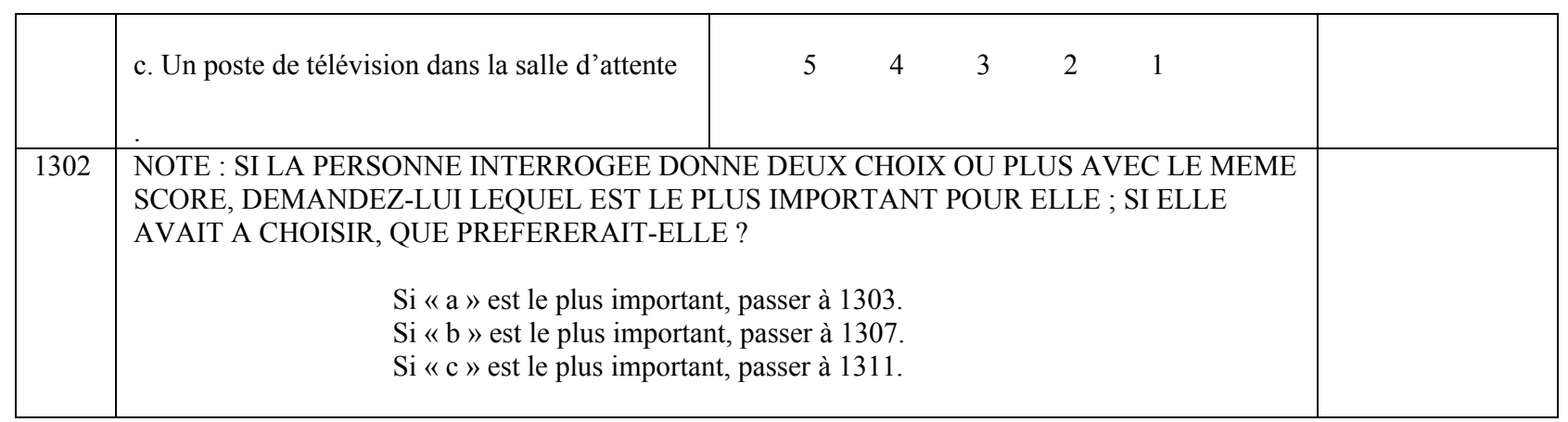

\begin{tabular}{|c|c|c|c|c|}
\hline 1303 & $\begin{array}{l}\text { Vous avez classé des chaises plus } \\
\text { confortables comme étant l'amélioration la } \\
\text { plus importante. Seriez-vous prêt à payer } 2 \text { de } \\
\text { plus, soit MONTANT PAYE + 2, pour votre } \\
\text { prochaine consultation si le dispensaire } \\
\text { effectuait cette amélioration? }\end{array}$ & $\begin{array}{l}\text { Oui } \\
\text { Non } \\
\text { Ne sait pas }\end{array}$ & $\begin{array}{c}1 \\
2 \\
99\end{array}$ & $\begin{array}{l}\text { passer à } 1305 \\
\text { passer à } 1305\end{array}$ \\
\hline 1304 & $\begin{array}{l}\text { Seriez-vous prêt à payer } 3 \text { de plus, soit } \\
\text { MONTANT PAYE }+\mathbf{3} \text {, pour votre prochaine } \\
\text { consultation si le dispensaire effectuait cette } \\
\text { amélioration? }\end{array}$ & $\begin{array}{l}\text { Oui } \\
\text { Non } \\
\text { Ne sait pas }\end{array}$ & $\begin{array}{c}1 \\
2 \\
99\end{array}$ & $\begin{array}{l}\text { passer à } 1306 \\
\text { passer à } 1306 \\
\text { passer à } 1306\end{array}$ \\
\hline 1305 & $\begin{array}{l}\text { Seriez-vous prêt à payer } 1 \text { de plus, soit } \\
\text { MONTANT PAYE + 1, pour votre prochaine } \\
\text { consultation si le dispensaire effectuait cette } \\
\text { amélioration? }\end{array}$ & $\begin{array}{l}\text { Oui } \\
\text { Non } \\
\text { Ne sait pas }\end{array}$ & $\begin{array}{c}1 \\
2 \\
99\end{array}$ & \\
\hline 1306 & $\begin{array}{l}\text { Quel est le prix le plus élevé que vous seriez } \\
\text { prêt à payer si cette amélioration était } \\
\text { apportée? }\end{array}$ & Montant & & \\
\hline 1307 & $\begin{array}{l}\text { Vous avez classé la climatisation comme étant } \\
\text { l'amélioration la plus importante. Seriez-vous } \\
\text { prêt à payer } 2 \text { de plus, soit MONTANT PAYE } \\
+\mathbf{2} \text {, pour votre prochaine consultation si le } \\
\text { dispensaire effectuait cette amélioration? }\end{array}$ & $\begin{array}{l}\text { Oui } \\
\text { Non } \\
\text { Ne sait pas }\end{array}$ & $\begin{array}{c}1 \\
2 \\
99\end{array}$ & $\begin{array}{l}\text { passer à } 1309 \\
\text { passer à } 1309\end{array}$ \\
\hline 1308 & $\begin{array}{l}\text { Seriez-vous prêt à payer } 3 \text { de plus, soit } \\
\text { MONTANT PAYE }+3 \text {, pour votre prochaine } \\
\text { consultation si le dispensaire effectuait cette } \\
\text { amélioration? }\end{array}$ & $\begin{array}{l}\text { Oui } \\
\text { Non } \\
\text { Ne sait pas }\end{array}$ & $\begin{array}{c}1 \\
2 \\
99\end{array}$ & $\begin{array}{l}\text { passer à } 1310 \\
\text { passer à } 1310 \\
\text { passer à } 1310\end{array}$ \\
\hline 1309 & $\begin{array}{l}\text { Seriez-vous prêt à payer } 1 \text { de plus, soit } \\
\text { MONTANT PAYE + 1, pour votre prochaine } \\
\text { consultation si le dispensaire effectuait cette } \\
\text { amélioration? }\end{array}$ & $\begin{array}{l}\text { Oui } \\
\text { Non } \\
\text { Ne sait pas }\end{array}$ & $\begin{array}{c}1 \\
2 \\
99\end{array}$ & \\
\hline 1310 & $\begin{array}{l}\text { Quel est le prix le plus élevé que vous seriez } \\
\text { prêt à payer si cette amélioration était } \\
\text { apportée? }\end{array}$ & Montant & & \\
\hline 1311 & $\begin{array}{l}\text { Vous avez classé la télévision comme étant } \\
\text { l'amélioration la plus importante. Seriez-vous } \\
\text { prêt à payer } 2 \text { de plus, soit MONTANT PAYE } \\
+2 \text {, pour votre prochaine consultation si le } \\
\text { dispensaire effectuait cette amélioration? }\end{array}$ & $\begin{array}{l}\text { Oui } \\
\text { Non } \\
\text { Ne sait pas }\end{array}$ & $\begin{array}{c}1 \\
2 \\
99\end{array}$ & $\begin{array}{l}\text { passer à } 1313 \\
\text { passer à } 1313\end{array}$ \\
\hline 1312 & $\begin{array}{l}\text { Seriez-vous prêt à payer } 3 \text { de plus, soit } \\
\text { MONTANT PAYE }+3 \text {, pour votre prochaine } \\
\text { consultation si le dispensaire effectuait cette } \\
\text { amélioration? }\end{array}$ & $\begin{array}{l}\text { Oui } \\
\text { Non } \\
\text { Ne sait pas }\end{array}$ & $\begin{array}{c}1 \\
2 \\
99\end{array}$ & $\begin{array}{l}\text { PASSER À } 1314 \\
\text { passer à } 1314 \\
\text { passer à } 1314\end{array}$ \\
\hline 1313 & $\begin{array}{l}\text { Seriez-vous prêt à payer } 1 \text { de plus, soit } \\
\text { MONTANT PAYE }+1 \text {, pour votre prochaine } \\
\text { consultation si le dispensaire effectuait cette }\end{array}$ & $\begin{array}{l}\text { Oui } \\
\text { Non } \\
\text { Ne sait pas }\end{array}$ & $\begin{array}{c}1 \\
2 \\
99\end{array}$ & \\
\hline
\end{tabular}




\begin{tabular}{|l|l|l|l|}
\hline 1314 & amélioration? & $\begin{array}{l}\text { Quel est le prix le plus élevé que vous seriez } \\
\text { prêt à payer si cette amélioration était } \\
\text { apportée? }\end{array}$ & Montant
\end{tabular}

\begin{tabular}{|l|l|}
\hline END & METTRE FIN A L'ENTRETIEN ET MERCIER LA PERSONNE INTERROGEE POUR
\end{tabular} VOUS AVOIR CONSACRE SON TEMPS A DONNER SES REPONSES. 


\section{Préservatifs \\ Acceptation de paiement \\ Modèle de questionnaire destiné aux hommes mariés \\ ou sexuellement actifs âgés de $\mathbf{1 5}$ à $\mathbf{4 5}$ ans}

(Basé sur une étude effectuée par Futures Group/INFO-STAT au Mali en 1999)

\section{ENQUETE AUPRES DES MENAGES SUR LES UTILISATEURS ACTUELS ET POTENTIELS DE PRÉSERVATIFS}

Bonjour ! Je m'appelle qui s'appelle . Je travaille pour un organisme de recherche J'aimerais vous poser quelques questions à propos des préservatifs, un produit qui peut être utilisé pour arrêter ou prévenir la grossesse, les maladies sexuellement transmissibles et le SIDA. Vous n'êtes pas obligé de participer à cet entretien, mais je vous serais très reconnaissant si vous acceptiez de répondre à quelques questions. Tout ce que vous direz restera confidentiel et personne ne saura comment vous aurez répondu personnellement aux questions que je vais vous poser.

Participation Oui-- $\rightarrow$ Continuer

Non-- $\rightarrow$ Fin de l'entretien

Questionnaire numéro :

Date :

Nom de l'enquêteur :
Région :
1. Bamako
2. Sikasso
3. Mopti

Zone: $\quad$ 1. Urbaine 2. Rurale

Bourg/village :

Quartier :

Nom de la personne interrogée :

Note : Questions basée sur les préservatifs vendus par paquet de trois 


\begin{tabular}{|c|c|c|}
\hline QUESTION & CODE REPONSE & PASSER A \\
\hline 1. Quel âge avez-vous? & $\frac{\mid}{(\operatorname{minim} .}=15$ ans, maxim. $=45$ ans $)$ & \\
\hline $\begin{array}{l}\text { 2. Situation de famille, activité } \\
\text { sexuelle }\end{array}$ & $\begin{array}{ll}\text { 1. } & \text { Marié (mariage consommé) } \\
\text { 2. } & \text { Vivant avec quelqu'un } \\
\text { 3. } & \text { Pas de relation, sexuellement } \\
& \text { actif } \\
\text { 4. } & \text { Pas de relation, pas } \\
& \text { sexuellement actif }\end{array}$ & \\
\hline $\begin{array}{l}\text { 3. Combien d'enfants vivants } \\
\text { avez-vous? }\end{array}$ & 1 & \\
\hline 4. Etes vous allé à l'école? & $\begin{array}{ll}\text { 1. } & \text { Oui } \\
\text { 2. } & \text { Non }\end{array}$ & $\rightarrow$ Passer à question 6 \\
\hline 5. Quel niveau avez-vous atteint? & $\begin{array}{ll}\text { 1. } & \text { Primaire } \\
\text { 2. } & \text { Secondaire } \\
\text { 3. } & \text { Lycée } \\
\text { 4. } & \text { Supérieur }\end{array}$ & \\
\hline 6. Quelle est votre profession? & $\begin{array}{ll}\text { 1. } & \text { Etudiant } \\
\text { 2. } & \text { Sans emploi } \\
\text { 3. } & \text { Employé de bureau } \\
\text { 4. } & \text { Marchand } \\
\text { 5. } & \text { Indépendant } \\
\text { 6. } & \text { Agriculteur } \\
\text { 7. } & \text { Eleveur } \\
\text { 8. } & \text { Pêcheur } \\
\text { 88. } & \text { Autre, } \\
\end{array}$ & \\
\hline 7. Chez vous, avez-vous : & 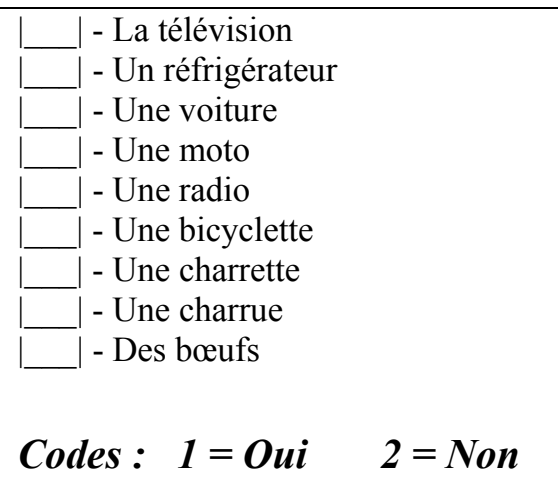 & \\
\hline
\end{tabular}


Enquêteur : Expliquer à la personne interrogée vous allez maintenant lui poser une série de question sur l'utilisation qu'elle fait de préservatifs.

\begin{tabular}{|c|c|c|}
\hline QUESTION & CODE REPONSE & PASSER A \\
\hline $\begin{array}{l}\text { 8. Avez-vous entendu parler des } \\
\text { préservatifs, un moyen de contrôle } \\
\text { des naissances et de prévention des } \\
\text { maladies utilisé par un homme? }\end{array}$ & $\begin{array}{ll}\text { 1. } & \text { Oui } \\
\text { 2. } & \text { Non }\end{array}$ & $\rightarrow$ Fin de l'entretien \\
\hline $\begin{array}{l}\text { 9. Vous êtes-vous jamais servi d'un } \\
\text { préservatif? }\end{array}$ & $\begin{array}{ll}\text { 1. } & \text { Oui } \\
\text { 2. } & \text { Non } \\
\end{array}$ & $\rightarrow$ Passer à question 27 \\
\hline $\begin{array}{l}\text { 10. Avez-vous utilisé un préservatif } \\
\text { au cours des } 3 \text { derniers mois? }\end{array}$ & $\begin{array}{ll}\text { 1. } & \text { Oui } \\
\text { 2. } & \text { Non } \\
\end{array}$ & $\rightarrow$ Passer à question 23 \\
\hline $\begin{array}{l}\text { 11. La dernière fois que vous avez } \\
\text { utilisé un préservatif, quelle en était } \\
\text { la marque? (montrer l'emballage } \\
\text { si nécessaire, une seule réponse) }\end{array}$ & $\begin{array}{ll}\text { 1. } & \text { Protector } \\
\text { 88. } & \text { Autre que } \\
\text { Protector, } \\
\text { 99. Je ne m'en souviens pas }\end{array}$ & \\
\hline $\begin{array}{l}\text { 12. Quelle(s) autre(s) marque(s) } \\
\text { avez-vous utilisé au cours des trois } \\
\text { derniers mois? (plusieurs } \\
\text { réponses possibles) }\end{array}$ & $\begin{array}{ll}\text { 1. } & \text { Aucune autre } \\
\text { 88. Autre, } \\
\text { 99. Je ne m'en souviens pas }\end{array}$ & \\
\hline $\begin{array}{l}\text { 13. La dernière fois que vous avez } \\
\text { utilisé un préservatif, où vous l'êtes- } \\
\text { vous procuré? }\end{array}$ & $\begin{array}{ll}\text { 1. } & \text { Magasin } \\
\text { 2. } & \text { Pharmacie } \\
\text { 3. } & \text { Centre de santé } \\
\text { communautaire } \\
\text { 4. Autre centre de santé } \\
\text { 5. Agent de distribution } \\
\text { communautaire } \\
\text { 88. Autre } \\
\text { 99. Ne sait pas/Ne s'en } \\
\text { souvient pas } \\
\end{array}$ & \\
\hline $\begin{array}{l}\text { 14.La dernière fois que vous avez } \\
\text { acheté des préservatifs, combien de } \\
\text { paquets de } 3 \text { avez-vous acheté? }\end{array}$ & & \\
\hline $\begin{array}{l}\text { 15. La dernière fois que vous avez } \\
\text { acheté des préservatifs, combien } \\
\text { avez-vous payé pour un paquet de } \\
3 \text { ? } \\
\text { Enquêteur : Si la quantité } \\
\text { mentionnée à la question } 14 \text { est } \\
\text { supérieure à } 3 \text {, aider la personne } \\
\text { interrogée à calculer le montant } \\
\text { payé par paquet de } 3 \text { en divisant } \\
\text { le total payé par le nombre de } \\
\text { paquets achetés. }\end{array}$ & $\begin{array}{l}\text { Code } 0 \mathrm{~F}=\text { rien/gratuitement } \\
\text { Code } 99=\text { Je ne sais pas }\end{array}$ & \\
\hline
\end{tabular}




\begin{tabular}{|c|c|c|}
\hline QUESTION & CODE REPONSE & PASSER A \\
\hline $\begin{array}{l}\text { 16. Vous avez dit que vous avez } \\
\text { payé (donner le prix } \\
\text { mentionné à la question 15) pour } \\
\text { le dernier paquet de } 3 \text { préservatifs } \\
\text { que vous avez acheté. Si le prix était } \\
\text { augmenté et passait à (Montant payé } \\
+50 \text { F), continueriez-vous à acheter } \\
\text { ces préservatifs? }\end{array}$ & $\begin{array}{ll}\text { 1. } & \text { Oui } \\
\text { 2. } & \text { Non }\end{array}$ & $\rightarrow$ Passer à question 18 \\
\hline $\begin{array}{l}\text { 17. Si le prix était augmenté et } \\
\text { passait à (Montant payé }+100 \mathrm{~F}) \text {, } \\
\text { continueriez-vous à acheter ces } \\
\text { préservatifs? }\end{array}$ & $\begin{array}{ll}\text { 1. } & \text { Oui } \\
\text { 2. } & \text { Non }\end{array}$ & $\begin{array}{l}\rightarrow \text { Passer à question } 19 \\
\rightarrow \text { Passer à question } 19\end{array}$ \\
\hline $\begin{array}{l}\text { 18. Si le prix était augmenté et } \\
\text { passait à (Montant payé }+25 \mathrm{~F}) \text {, } \\
\text { continueriez-vous à acheter ces } \\
\text { préservatifs? }\end{array}$ & $\begin{array}{ll}\text { 1. } & \text { Oui } \\
\text { 2. } & \text { Non }\end{array}$ & \\
\hline $\begin{array}{l}\text { 19. Quel est le prix le plus élevé que } \\
\text { vous seriez prêt à payer pour un } \\
\text { paquet de } 3 \text { préservatifs? }\end{array}$ & F CFA & \\
\hline $\begin{array}{l}\text { 20. Si le prix des préservatifs à } \\
\text { (donner le lieu mentionné à } \\
\text { la question 13) dépasse ce que vous } \\
\text { êtes prêt à payer ou avez la capacité } \\
\text { de payer, que feriez-vous? } \\
\text { (donner les réponses) }\end{array}$ & $\begin{array}{l}\text { 1. Chercher une marque moins } \\
\text { chère au même endroit } \\
\text { 2. Chercher la même marque à } \\
\text { un endroit qui la vend moins } \\
\text { cher } \\
\text { 3. Chercher une marque moins } \\
\text { chère et un endroit moins } \\
\text { cher } \\
\text { 4. Arrêter de s'en servir } \\
\text { 88. Autre, }\end{array}$ & \\
\hline $\begin{array}{l}\text { 21. Quelle est la principale raison } \\
\text { pour laquelle vous utilisez des } \\
\text { préservatifs? }\end{array}$ & $\begin{array}{ll}\text { 1. } & \text { Prévention de la grossesse } \\
\text { 2. } & \text { Protection contre } \\
\text { MST/VIH/SIDA } \\
\text { 3. Les deux } \\
\end{array}$ & \\
\hline $\begin{array}{l}\text { 22. La dernière fois que vous avez } \\
\text { utilisé un préservatif, avec qui } \\
\text { l'avez-vous fait? }\end{array}$ & $\begin{array}{l}\text { 1. Ma femme } \\
\text { 2. Une partenaire régulière qui } \\
\text { n'est pas ma femme } \\
\text { 3. Une partenaire occasionnelle } \\
\text { 88. Autre, } \\
\text { 99. Pas de réponse }\end{array}$ & $\rightarrow$ Fin de l'entretien \\
\hline $\begin{array}{l}\text { 23. Il y a combien de temps que } \\
\text { vous avez utilisé un préservatif pour } \\
\text { la dernière fois? }\end{array}$ & $\begin{array}{ll}\text { 1. } & \text { Entre } 4 \text { et } 6 \text { mois } \\
\text { 2. } & \text { Entre } 7 \text { et } 12 \text { mois } \\
\text { 3. } & \text { Plus d'un an } \\
\text { 99. } & \text { Je ne m'en souviens pas }\end{array}$ & $\begin{array}{l}\rightarrow \text { Passer à question } 27 \\
\rightarrow \text { Passer à question } 27\end{array}$ \\
\hline $\begin{array}{l}\text { 24. Pour quelle raison avez-vous } \\
\text { utilisé un préservatif dans le passé ? }\end{array}$ & $\begin{array}{ll}\text { 1. } & \text { Prévention de la grossesse } \\
\text { 2. } & \text { Protection contre } \\
\text { MST/VIH/SIDA } \\
\text { 3. Les deux } \\
\end{array}$ & \\
\hline
\end{tabular}




\begin{tabular}{|c|c|c|}
\hline QUESTION & CODE REPONSE & PASSER A \\
\hline $\begin{array}{l}\text { 25. Avec qui avez-vous utilisé un } \\
\text { préservatif pour la dernière fois? }\end{array}$ & $\begin{array}{l}\text { 1. Ma femme } \\
\text { 2. Une partenaire régulière qui } \\
\text { n'est pas ma femme } \\
\text { 3. Une partenaire occasionnelle } \\
\text { 88. Autre, } \\
\text { 99. Pas de réponse }\end{array}$ & \\
\hline $\begin{array}{l}\text { 26. Où achetez-vous normalement } \\
\text { des préservatifs? }\end{array}$ & $\begin{array}{ll}\text { 1. } & \text { Magasin } \\
\text { 2. } & \text { Pharmacie } \\
\text { 3. Centre de santé } \\
\text { communautaire } \\
\text { 4. Autre centre de santé } \\
\text { 5. Agent de distribution } \\
\text { communautaire } \\
\text { 88. Autre, } \\
\text { 99. Ne sait pas/ Ne s'en souvient } \\
\text { pas }\end{array}$ & \\
\hline $\begin{array}{l}\text { 27. Pour quelle raison avez-vous } \\
\text { arrêté d'utiliser des préservatifs? }\end{array}$ & $\begin{array}{l}\text { 1. Pas les moyens / trop cher } \\
\text { 2. Veut des enfants } \\
\text { 3. N'aime plus les utiliser, } \\
\text { raison } \\
\text { 4. Pas faciles à obtenir } \\
\text { 5. Pas sexuellement actif } \\
\text { 88. Autre, spécifier }\end{array}$ & \\
\hline $\begin{array}{l}\text { 28. Utiliseriez-vous des préservatifs } \\
\text { à l'avenir? }\end{array}$ & $\begin{array}{ll}\text { 1. } & \text { Oui } \\
\text { 2. } & \text { Non } \\
\text { 99. } & \text { Ne sait pas }\end{array}$ & $\begin{array}{l}\rightarrow \text { Passer à question } 34 \\
\rightarrow \text { Fin de l'entretien }\end{array}$ \\
\hline $\begin{array}{l}\text { 29. Achèteriez-vous un paquet de } \\
3 \text { préservatifs pour } 100 \mathrm{~F} \text { ? }\end{array}$ & $\begin{array}{ll}\text { 1. } & \text { Oui } \\
\text { 2. } & \text { Non }\end{array}$ & $\rightarrow$ Passer à question 31 \\
\hline $\begin{array}{l}30 \text {. Achèteriez-vous un paquet de } \\
3 \text { préservatifs pour } 150 \mathrm{~F} \text { ? }\end{array}$ & $\begin{array}{ll}\text { 1. } & \text { Oui } \\
\text { 2. } & \text { Non }\end{array}$ & $\begin{array}{l}\rightarrow \text { Passer à question } 32 \\
\rightarrow \text { Passer à question } 32\end{array}$ \\
\hline $\begin{array}{l}\text { 31. Achèteriez-vous un paquet de } \\
3 \text { préservatifs pour } 75 \mathrm{~F} \text { ? }\end{array}$ & $\begin{array}{ll}\text { 1. } & \text { Oui } \\
\text { 2. } & \text { Non }\end{array}$ & $\begin{array}{l}\rightarrow \text { Passer à question } 32 \\
\rightarrow \text { Passer à question } 32\end{array}$ \\
\hline $\begin{array}{l}\text { 32. Quel est le prix le plus élevé que } \\
\text { vous seriez prêt à payer pour un } \\
\text { paquet de } 3 \text { préservatifs? }\end{array}$ & $\begin{array}{l}\text { F CFA } \\
\mathbf{0 = \text { Pas d'achat }}\end{array}$ & \\
\hline $\begin{array}{l}\text { 33. Quelle est la raison principale } \\
\text { pour laquelle vous utiliseriez des } \\
\text { préservatifs à l'avenir? }\end{array}$ & $\begin{array}{l}\text { 1. Prévention de la grossesse } \\
\text { 2. Protection contre } \\
\text { MST/VIH/SIDA } \\
\text { 3. Les deux }\end{array}$ & \\
\hline
\end{tabular}




\begin{tabular}{|l|l|l|}
\hline QUESTION & CODE REPONSE & PASSER A \\
\hline 34. Pour quelle raison n'utiliseriez- & 1. Religion & \\
vous pas de préservatifs ? & 2. Plaisir limité & \\
(Entourez toutes les réponses & 3. Les préservatifs encouragent & \\
données d'un cercle) & la débauche & \\
& 4. Utilise une autre méthode de & \\
& 5. Prianing familial & \\
& 6. Accès peu facile aux & \\
& 7. Opréservatifs \\
& 8. Pas de partenaire sexuel & \\
& 88. Autre, & \\
\hline
\end{tabular}

\section{FIN DE L'ENTRETIEN}

Enquêteur : Vérifier que vous n'avez pas oublié de question. Remercier la personne interrogée pour sa coopération. 


\section{ANNEXE II \\ MODELE DE MANUEL DE L'ENQUETEUR/ENQUETRICE}

Instructions relatives à la façon de remplir le formulaire sur les augmentations de prix et questions apparentées figurant dans le modèle de questionnaire : Utilisateurs actuels et potentiels de services de clinique (entretien d'interception)

\section{MODELE DE MANUEL DE CODAGE}

Instructions relatives au codage du modèle de questionnaire : Utilisateurs actuels et potentiels de services de clinique (entretien d'interception) 


\section{Acceptation de paiement Modèle de manuel de l'enquêteur/enquêtrice}

Instructions relatives à la façon de remplir le formulaire sur les augmentations de prix et questions apparentées figurant dans le modèle de questionnaire : Utilisateurs actuels et potentiels de services de clinique (entretien d'interception)

\section{INSTRUCTIONS D'ORDRE GENERAL}

A. Toujours utiliser un $\underline{\text { crayon }}$ (mine douce ou moyenne).

B. Ecrire en caractère d'imprimerie toutes les lettres et tous les chiffre de façon claire.

C. Remplir toutes les questions qui s'appliquent à la personne interrogée.

D. Ne rien laisser en blanc. Si la réponse à une question est « pas de réponse », entourer le 99 pour « Pas de réponse ». Si un nombre est zéro, marquer 00.

E. Il faut noter les commentaires quand ils sont faits. Informer dès que possible votre supérieur (e) de tout commentaire ou de toute question.

F. Le/la supérieur(e) doit faire une note de tous les commentaires et de toutes les questions et en rendre compte au coordinateur (à la coordinatrice) de l'étude.

G. Toutes les instructions destinées à l'enquêteur/enquêtrice doivent être rédigées en majuscules et en caractères gras. Ces instructions ne doivent pas être lues à la personne interrogée. Par exemple :

\begin{tabular}{|c|c|c|c|}
\hline Q. & QUESTION & CODE REPONSE & PASSER A \\
\hline 207 & $\begin{array}{l}\text { Quel est le revenu mensuel TOTAL de votre } \\
\text { famille? } \\
\text { DEMANDER TOUTES LES SOURCES DE } \\
\text { REVENU (LOYER, CADEAUX, ETC.) } \\
\text { LE MONTANT DOIT ETRE EGAL OU } \\
\text { SUPERIEUR A (Q203 + Q205) }\end{array}$ & Total TOUTES SOURCES & \\
\hline
\end{tabular}

\section{STRUCTURE DU QUESTIONNAIRE}

\section{A. Organisation}

Le questionnaire est organisé en rangées et colonnes. Par exemple :

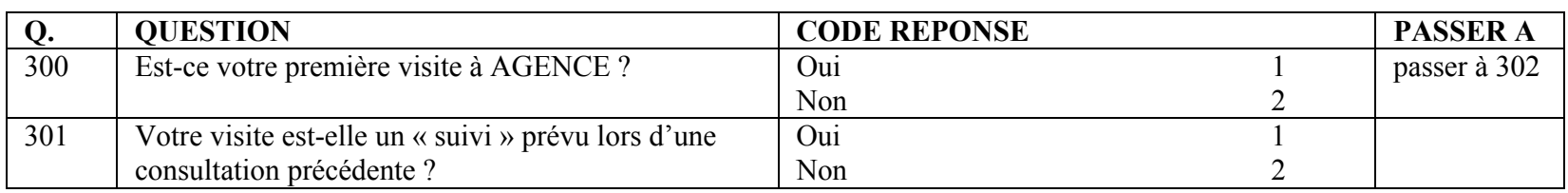


La première colonne comporte le numéro de la question. La deuxième colonne comporte la question à lire à la personne interrogée ainsi que toute instruction destinée à l'enquêteur/enquêtrice. Ces instructions sont en caractères gras.

La troisième colonne comporte les réponses et leurs codes. S'assurer que le code approprié est entouré d'un cercle (par exemple, pour la question 300 le code pour « Oui » est le 1 et le code pour « Non » est le 2). Pour les questions ouvertes, s'assurer que la réponse est enregistrée mot à mot. Par exemple :

\begin{tabular}{|l|l|ll|l|}
\hline Q. & QUESTION & CODE REPONSE & PASSER A \\
\hline 201 & Travaillez-vous en dehors de la maison? & Oui & 1 & passer à 204 \\
& & Non & 2 & \\
\hline 202 & Que faites-vous? & Emploi & & \\
& & & & \\
\hline
\end{tabular}

La quatrième colonne comporte les instructions « passer à », si c'est le cas.

\section{B. Passer à}

Les instructions données dans la quatrième colonne, intitulée «PASSER », indiquent à l'enquêteur/enquêtrice à quelle question il faut passer. Différentes réponses données dans la troisième colonne par la personne interrogée la font souvent passer à d'autres questions.

Par exemple :

\begin{tabular}{|l|l|ll|l|}
\hline Q. & QUESTION & CODE REPONSE & PASSER A \\
\hline 302 & Quel est le principal service dont vous avez & Gynécologie & passer à 400 \\
& bénéficié aujourd'hui ? & Contraceptif injectable & 2 & Passer à 500 \\
& & Pose stérilet & 3 & Passer à 600 \\
& & Examen stérilet & 4 & passer à 700 \\
& & Pilule & 5 & passer à 800 \\
& & Soins prénatals & 6 & passer à 900 \\
& & & & \\
& & Autre : spécifier & 88 & passer à FIN \\
\hline
\end{tabular}

Dans le cas de la question 302, les personnes interrogées qui déclarent être venues au dispensaire pour un service de gynécologie passent à la question 400. Celles qui répondent être venues pour une pose de stérilet passent à la 600 .

Ces manœuvres sont probablement la partie la plus difficile de tout questionnaire pour l'enquêteur/enquêtrice. Il faut faire preuve de grand soin à respecter les instructions qui s'y rattachent. Il y aura perte de données et confusion pour les personnes interrogées si vous ratez un « passer à ». Les supérieur(e)s doivent soigneusement passer en revue tous les questionnaires afin de rechercher toute erreur dans ce domaine.

\section{Dates}

Lorsqu'une date est demandée (par exemple, celle de l'entretien), toujours utiliser deux chiffres. Par exemple, si la date est le 6 mai 2000, elle doit être notée comme suit : jour $\underline{06}$ mois $\underline{05}$ année $\underline{00}$.

\section{Argent}

Ne pas utiliser de décimales. Par exemple, écrire 25 pesos au lieu de 25,00. Si la personne interrogée donne un chiffre qui n'est pas entier, arrondir au chiffre inférieur. Par exemple, une réponse de 10,75 pesos doit être arrondie à 10 pesos et un chiffre inférieur à un peso doit être arrondi à 00 . Les sommes inférieures à 10 doivent être précédée d'un zéro. Par exemple, 5 pesos doit être écrit 05 pesos. 


\section{SELECTION DES FEMMES A INTERROGER}

A. N'interroger que les femmes qui se sont présentées pour un des services mentionnés sur le questionnaire. Ce sont les suivants :

- Gynécologie

- Contraceptif injectable

- Pose de stérilet

- Examen stérilet

- Pilule

- Soins prénatals

B. Les entretiens doivent être menés dans le dispensaire pendant les heures d'ouverture.

C. La sage-femme doit présenter la cliente à l'enquêtrice à la fin de la consultation et lorsque la cliente a reçu tous les services et effectué tous les achats.

D. L'enquêtrice doit remplir le numéro du questionnaire, le code du dispensaire, le nom de l'enquêtrice, la date et l'heure de début de l'entretien avant de commencer ce dernier. Le code du dispensaire doit être fourni à l'enquêtrice sur une carte ou une feuille de papier séparée.

\section{INTRODUCTION}

Les premières tâches de l'enquêtrice consistent à se présenter, à informer la personne interrogée potentielle des objectifs, risques et avantages de la recherche et à obtenir le consentement de la personne à interroger. La plupart des organismes de recherche exigent que la personne interrogée donne son consentement en toute connaissance de cause, qui trouve son origine dans la recherche médicale, qui comprend des risques et des avantages. Les formulaires de consentement doivent utiliser des phrases simples et des déclarations directes, appropriées au niveau d'alphabétisation ou de scolarisation des personnes interrogées. Il faut donner au participant l'occasion de poser des questions et il doit être bien informé avant de signer le formulaire de consentement. Les principaux éléments d'un consentement en toute connaissance de cause sont les suivants :

1. But de l'étude.

2. Risques encourus par le participant à l'étude, y compris les risques physiques, sociaux et émotionnels.

3. Avantages que le participant peut tirer de l'étude, y compris, si nécessaire, une déclaration selon laquelle il n'en existe pas.

4. Informations relatives à la confidentialité, y compris l'utilisation d'identificateurs et l'accès à des données personnelles.

5. Où aller à tout moment en cas de question ou de problème.

6. Informations concernant le retrait de l'étude. Le participant a le droit de décider de ne plus en faire partie à quelque moment que ce soit, sans pénalisation.

7. Garantie que les services sont disponibles, que le participant potentiel décide ou non de prendre part à l'étude ou s'il décide de ne plus y participer.

8. Informations sur les procédures suivies et la durée de l'étude.

Le formulaire doit être sur une feuille de papier différente des autres. Il faut noter que l'enquêtrice doit pouvoir relier le formulaire de consentement au questionnaire approprié en leur donnant un numéro. Pour la collecte de données ne présentant qu'un risque peu élevé, un consentement oral suffit, mais le formulaire doit être signé par l'enquêtrice aux fins de documentation.

Chaque organisation a sa façon propre d'obtenir un consentement en toute connaissance de cause. Le questionnaire comporte un exemple de consentement en toute connaissance de cause. Un exemple d'enquête WTP est donné ci-après. 
INSTRUCTIONS DESTINEE A L'ENQUETRICE : Il faut lire la déclaration suivante à toute personnes interrogée potentielle. Puis-je vous demander un moment, s'il vous plait ? L'AGENCE désire continuer à vous offrir des services opportuns et de haute qualité. Pour cela, il est nécessaire qu'elle augmente les prix de certains services. Nous voudrions savoir comment ces changements de prix vous affecteront. Il n'y a pas de bonne ou de mauvaise réponse, alors soyez honnête et dites-nous ce qui est vrai pour vous. Les informations que nous collectons ne serviront qu'à des fins de planification et votre participation ne comporte ni risques, ni avantages personnels. Vous n'avez pas besoin de me donner votre nom et votre adresse. Tout ce que vous me direz restera confidentiel et le seul identificateur qui figure sur le questionnaire sera un numéro et non pas votre nom. Personne, y compris le personnel de ce dispensaire, ne saura ce que vous nous aurez dit personnellement personne ne saura votre nom. Que vous acceptiez ou non de prendre part à cette étude, vous pourrez toujours avoir recours aux services. L'entretien prend environ quinze minutes. Vous pouvez l'abandonner n'importe quand sans conséquences. Avez-vous des questions ? Voudriez-vous y participer? Si vous voulez en savoir davantage sur cette étude, veuillez contacter à
1. Participation
OUI (effectuer l'entretien)
NON (remercier la personne et lui souhaiter une bonne journée)

L'enquêtrice conservera tous les questionnaires, que la personne interrogée décide ou non de participer à l'enquête.

\section{INSTRUCTIONS POUR REMPLIR CHAQUE QUESTION}

Le manuel de l'enquêtrice doit contenir des instructions complètes sur la façon de remplir chaque question du questionnaire. Par exemple :

Question 302 : C'est la question la plus importante du questionnaire. Elle vous dirige vers les différentes sections qu'il faut remplir, en se basant sur le principal service dont la cliente a bénéficié.

\begin{tabular}{|l|l|ll|l|}
\hline Q. & QUESTION & CODE REPONSE & & PASSER A \\
\hline 302 & Quel est le principal service dont vous avez & Gynécologie & 1 & passer à 400 \\
& bénéficié aujourd'hui ? & Contraceptif injectable & 2 & passer à 500 \\
& & Pose stérilet & 3 & passer à 600 \\
& & Examen stérilet & 4 & passer à 700 \\
& & Pilule & 5 & passer à 800 \\
& & Soins prénatals & 6 & passer à 900 \\
& & & & \\
& & Autre : spécifier & 88 & passer à FIN \\
\hline
\end{tabular}

En se basant sur la réponse à cette question, il faut absolument que l'enquêtrice passe à la section suivante correcte. Une fois que cela est fait, elle doit poser toutes les questions qui y figurent.

Si la femme a obtenu deux services ou plus, l'enquêtrice doit aller plus loin en demandant «Quel est le principal service dont vous avez bénéficié aujourd'hui ? » Dans tous les cas, la personne interrogée doit identifier un seul service principal. Il faut que ce soit la personne interrogée qui identifie ce service et non pas l'enquêtrice.

\section{QUESTIONS RELATIVES A L'ACCEPTATION DE PAIEMENT}

Le but de cette enquête est de déterminer à quel point les clients sont prêts à payer pour les services qui y sont mentionnés. la première tâche de l'enquêtrice consiste à déterminer quel montant la personne interrogée vient de payer pour le service dont elle a bénéficié. Une fois arrivé à la section appropriée, le questionnaire demande à l'enquêtrice de dire à la cliente quel est le prix actuel du service et de bien souligner qu'il ne s'agit là que du prix du principal service proprement dit et que le montant total qu'elle a payé peut avoir été plus élevé. On demande ensuite à la cliente si elle a payé le prix actuel. 


\begin{tabular}{|l|l|l|c|}
\hline Q. & QUESTION & CODE REPONSE & PASSER A \\
\hline 400 & $\begin{array}{l}\text { LIRE A LA CLIENTE : } \\
\text { Le prix actuel d'une consultation gynécologique est de I/10. Il ne s'agit que du prix de la } \\
\text { consultation et votre coût total a pu être plus élevé si d'autres analyses recommandées par le } \\
\text { personnel ont été effectuées. }\end{array}$ & \\
\hline 401 & $\begin{array}{l}\text { Avez-vous payé le prix mentionné pour la } \\
\text { consultation? }\end{array}$ & $\begin{array}{l}\text { Oui } \\
\text { Non }\end{array}$ & 1 \\
\hline
\end{tabular}

Si la cliente répond par Non à la question 401, l'enquêtrice a pour instruction de lui en demander la raison.

L'enquêtrice doit alors déterminer si la différence est due au coût d'autres services qui ont été achetés, à un solde dû à une visite précédente ou si la cliente a bénéficié d'une remise parce qu'elle n'avait pas les moyens de payer le prix demandé. Il peut alors falloir poser la question au médecin ou au caissier du dispensaire. S'il est établi que la cliente est trop pauvre pour payer le prix demandé, l'enquêtrice la remerciera et mettra fin à l'entretien. Si la raison pour laquelle le prix indiqué par la cliente est différent de celui qui est normalement appliqué est dû à une confusion en raison de multiples achats ou du paiement d'un solde dû, l'enquêtrice doit noter le prix normal et poursuivre l'entretien.

\begin{tabular}{|l|l|l|l|}
\hline Q. & QUESTION & CODE REPONSE & PASSER A \\
\hline 402 & Combien avez-vous payé ? & Montant : & \\
\hline 403 & $\begin{array}{l}\text { Pourquoi avez-vous payé davantage/moins que le } \\
\text { prix mentionné ? }\end{array}$ & Raison : & \\
& $\begin{array}{l}\text { DETERMINER SI LA DIFFERENCE DE } \\
\text { PRIX EST DUE AU REGLEMENT } \\
\text { D'AUTRES SERVICES. SI OUI, } \\
\text { POURSUIVRE L'ENTRETIEN. SI NON, } \\
\text { ENREGISTREZ LA RAISON ET METTRE } \\
\text { FIN A L'ENTRETIEN. }\end{array}$ & & \\
\end{tabular}

La seconde tâche consiste à lire à la cliente la déclaration suivante :

\section{LIRE A LA CLIENTE :}

J'aimerais maintenant vous poser certaines questions à propos de votre réaction à des changements potentiels de prix pour cette consultation. En répondant à ces questions, gardez ce qui suit à l'esprit :

1. Partez de l'hypothèse que votre revenu restera identique, même en cas de changement de prix.

2. Il existe d'autres possibilités d'obtention des services (ministère de la Santé, clinique privée, par exemple).

Il est essentiel que la cliente comprenne bien les hypothèses de départ indiquées ci-dessus.

La série de questions relatives à l'acceptation de paiement (WTP). Une fois que l'enquêtrice a vérifié que la cliente a payé le prix actuel et a lu les hypothèses de départ mentionnées ci-dessus, elle continue en posant les questions relatives à l'acceptation de paiement. Cette série de questions consiste en deux questions portant sur des augmentations de prix prédéterminées et une troisième, ouverte, qui permet de déterminer le montant maximum que la femme serait prête à payer pour le service.

La première chose à faire lorsque l'on pose les questions relatives à l'acceptation de paiement est de dire à la femme interrogée de partir de l'hypothèse que le prix du service sera augmenté d'un montant donné, par exemple de I/5.

\begin{tabular}{|c|c|c|c|c|}
\hline Q. & QUESTION & CODE REPONSE & & PASSER A \\
\hline 405 & $\begin{array}{l}\text { Supposez que le prix d'une consultation } \\
\text { gynécologique passe de I/5 à } \mathrm{I} / 15 \text {. Utiliseriez-vous } \\
\text { l'AGENCE pour vos consultations de gynécologie } \\
\text { si le prix était de I/15? }\end{array}$ & $\begin{array}{l}\text { Oui } \\
\text { Non } \\
\text { Ne sait pas }\end{array}$ & $\begin{array}{l}1 \\
2 \\
99\end{array}$ & $\begin{array}{l}\text { passer à } 407 \\
\text { passer à } 407\end{array}$ \\
\hline
\end{tabular}

Si la cliente est prête à payer une augmentation de I/5, on continue avec la question suivante (406) afin de déterminer si elle est prête à payer un prix encore plus élevé. 


\begin{tabular}{|l|l|ll|l|}
\hline Q. & QUESTION & CODE REPONSE & PASSER A \\
\hline 406 & Supposez que le prix d'une consultation & Oui & passer à 408 \\
& gynécologique augmente encore davantage - de & Non & 2 & passer à 408 \\
& $\begin{array}{l}\text { I/10 à I/20. Utiliseriez-vous l'AGENCE pour vos } \\
\text { consultations de gynécologie si le prix était de } \\
\text { I/20? }\end{array}$ & Ne sait pas & 99 & passer à 408 \\
& & \\
\hline
\end{tabular}

Si la cliente n'est pas prête à payer une augmentation de I/5 à la question 405, on passe à la question 407 afin de déterminer si elle est prête à payer une augmentation moins élevée du prix.

\begin{tabular}{|l|l|ll|l|}
\hline Q. & QUESTION & CODE REPONSE & PASSER A \\
\hline 407 & Supposez que l'augmentation de prix soit & Oui & 1 & \\
& inférieure au montant précédent. Supposez que le & Non & 2 & \\
& prix d'une consultation gynécologique augmente & Ne sait pas & 99 & \\
& $\begin{array}{l}\text { de I/2 à I/12. Utiliseriez-vous l'AGENCE pour vos } \\
\text { consultations de gynécologie si le prix était de } \\
\text { I/12? }\end{array}$ & & \\
\hline
\end{tabular}

Veuillez noter qu'il y a un total de trois questions fermées concernant l'acceptation de paiement, mais aucune personne interrogée ne s'en voit poser plus de deux.

On pose à toutes les clientes, quelles qu'aient été leurs réponses aux questions précédentes, la question ouverte suivante sur le prix maximum qu'elles seraient prêtes à payer.

\begin{tabular}{|l|l|l|l|}
\hline Q. & QUESTION & CODE REPONSE & PASSER A \\
\hline 408 & $\begin{array}{l}\text { Quel est le prix le plus élevé que vous seriez prête } \\
\text { à payer pour des services gynécologiques de cette } \\
\text { AGENCE? }\end{array}$ & Montant & \\
\hline
\end{tabular}

Finalement, nous demandons ce que la femme ferait si le prix du service était augmenté au point où elle ne serait pas prête à ou dans l'incapacité de le payer (409 et 410). Dans le cas des moyens de contraception, « Passer à un moyen moins cher » est ajouté en tant que réponse possible.

\begin{tabular}{|c|c|c|c|c|}
\hline Q. & QUESTION & CODE REPONSE & & PASSER A \\
\hline 409 & $\begin{array}{l}\text { Si l'AGENCE augmentait le prix des services } \\
\text { gynécologie au-delà que ce que vous prête à } \\
\text { payer ou capable de payer, que feriez-vous? }\end{array}$ & $\begin{array}{l}\text { S'en passer } \\
\text { Aller ailleurs } \\
\text { Ne sait pas } \\
\text { Autre : spécifier }\end{array}$ & $\begin{array}{l}1 \\
2 \\
99 \\
88\end{array}$ & $\begin{array}{l}\text { passer à } 1000 \\
\text { passer à } 410 \\
\text { passer à } 1000 \\
\text { passer à } 1000\end{array}$ \\
\hline 410 & $\begin{array}{l}\text { Où iriez-vous? } \\
\text { NE PAS DONNER LES CHOIX } \\
\text { DONNER LE CODE DE TOUS CEUX QUI } \\
\text { SONT MENTIONNES }\end{array}$ & $\begin{array}{l}\text { Secteur public } \\
\text { Autre ONG } \\
\text { Secteur privé } \\
\text { Pharmacie } \\
\text { Autre : spécifier } \\
\text { Je ne sais pas }\end{array}$ & $\begin{array}{l}1 \\
2 \\
3 \\
4 \\
88 \\
99\end{array}$ & $\begin{array}{l}\text { passer à } 1000 \\
\text { passer à } 1000 \\
\text { passer à } 1000 \\
\text { passer à } 1000 \\
\text { passer à } 1000 \\
\text { passer à } 1000\end{array}$ \\
\hline
\end{tabular}

A la fin de cette section, l'enquêtrice trouvera des instructions lui demandant de passer à la section 1000 de l'entretien et au reste du questionnaire (acceptation de paiement pour les utilisateurs potentiels et acceptation de paiement pour l'amélioration des accommodements et de la qualité). Ces questions sont posées à toutes les personnes interrogées qui ont fini la série de questions relatives à l'acceptation de paiement. 
Santé de la reproduction

Acceptation de paiement

Modèle de manuel de codage

Questionnaire modèle pour

utilisateurs actuels et potentiels de services de clinique

(entretiens d'interception)

Population Council

Juin 2001

DICTIONNAIRE DONNEES ET GUIDE DE CODAGE

\begin{tabular}{|l|l|l|l|}
\hline Q. & VARIABLE : NOM & VARIABLE : SIGNIFICATION & VALEURS \\
\hline 1 & PARTIND & Indicateur de participation & 1 \\
& & & Oui Non \\
\hline 2 & QUESNO & Numéro du questionnaire & Code 4 chiffres : Fourchette 0001 - \\
\hline 3 & CLINICNO & Numéro du dispensaire & 101 Dispensaire 1 \\
& & & 102 Dispensaire 2 \\
& & & 103 Dispensaire 3 \\
\hline 4 & INTNAME & Nom de l'enquêteur/enquêtrice & 101 Nom 1 \\
& & & 102 Nom 2 \\
& & Date de l'entretien & JOUR - MOIS - ANNEE \\
\hline 5 & INTDATE & Heure de début de l'entretien & $24: 00$ \\
\hline 6 & TIMEBEG & Heure de fin de l'entretien & $24: 00$ \\
\hline 7 & TIMEEND & & \\
\hline
\end{tabular}

A. Questions démographiques

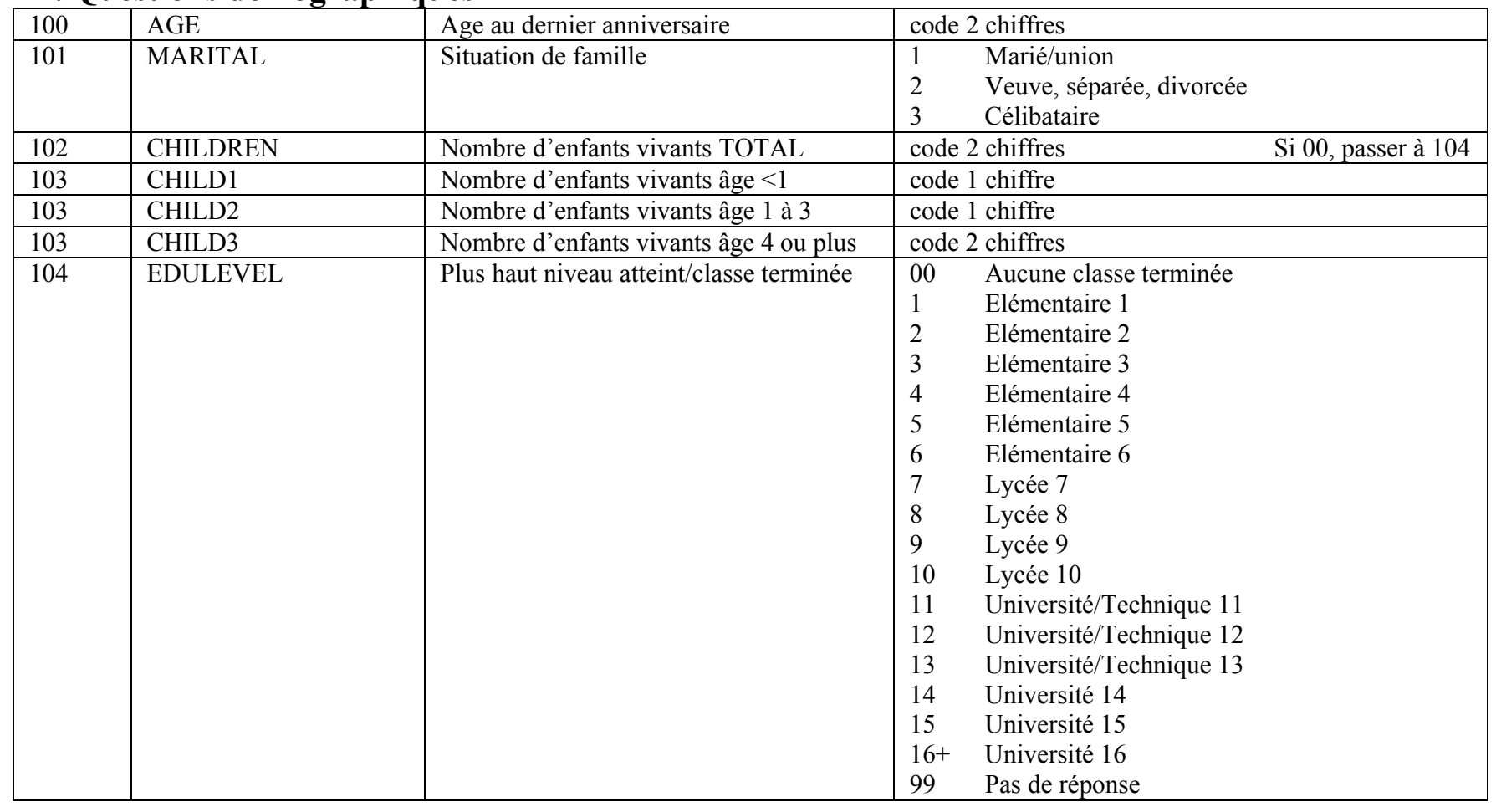


B. Questions relatives à la capacité de paiement

\begin{tabular}{|c|c|c|c|c|c|}
\hline 200 & HHELEC & Le ménage a-t-il l'électricité ? & $\begin{array}{l}1 \\
2 \\
99 \\
\end{array}$ & $\begin{array}{l}\text { Oui } \\
\text { Non } \\
\text { Pas de réponse } \\
\end{array}$ & \\
\hline 200 & HHWATER & Le ménage a-t-il l'eau courante? & $\begin{array}{l}1 \\
2 \\
99 \\
\end{array}$ & $\begin{array}{l}\text { Oui } \\
\text { Non } \\
\text { Pas de réponse } \\
\end{array}$ & \\
\hline 200 & HHFLTOIL & $\begin{array}{l}\text { Le ménage a-t-il des toilettes avec une } \\
\text { chasse d'eau? }\end{array}$ & $\begin{array}{l}1 \\
2 \\
99 \\
\end{array}$ & $\begin{array}{l}\text { Oui } \\
\text { Non } \\
\text { Pas de réponse } \\
\end{array}$ & \\
\hline 200 & HHRADIO & Le ménage a-t-il un poste de radio? & $\begin{array}{l}1 \\
2 \\
99 \\
\end{array}$ & $\begin{array}{l}\text { Oui } \\
\text { Non } \\
\text { Pas de réponse } \\
\end{array}$ & \\
\hline 200 & HHTV & Le ménage a-t-il un poste de télévision? & $\begin{array}{l}1 \\
2 \\
99 \\
\end{array}$ & $\begin{array}{l}\text { Oui } \\
\text { Non } \\
\text { Pas de réponse } \\
\end{array}$ & \\
\hline 200 & HHVCR & Le ménage a-t-il un magnétoscope ? & $\begin{array}{l}1 \\
2 \\
99 \\
\end{array}$ & $\begin{array}{l}\text { Oui } \\
\text { Non } \\
\text { Pas de réponse } \\
\end{array}$ & \\
\hline 200 & HHTELE & Le ménage a-t-il le téléphone? & $\begin{array}{l}1 \\
2 \\
99 \\
\end{array}$ & $\begin{array}{l}\text { Oui } \\
\text { Non } \\
\text { Pas de réponse }\end{array}$ & \\
\hline 200 & HHVEHCL & $\begin{array}{l}\text { Le ménage a-t-il une voiture ou une } \\
\text { camionnette? }\end{array}$ & $\begin{array}{l}1 \\
2 \\
99 \\
\end{array}$ & $\begin{array}{l}\text { Oui } \\
\text { Non } \\
\text { Pas de réponse } \\
\end{array}$ & \\
\hline 201 & WORKOUT & Travaillez-vous en dehors de la maison? & $\begin{array}{l}1 \\
2 \\
99 \\
\end{array}$ & $\begin{array}{l}\text { Oui } \\
\text { Non } \\
\text { Pas de réponse } \\
\end{array}$ & \\
\hline 202 & JOBTYPE & Que faites-vous? & En1 & istrer la réponse mot à mot & \\
\hline 203 & MOINC1 & $\begin{array}{l}\text { Revenu mensuel moyen de la personne } \\
\text { interrogée }\end{array}$ & & chiffres & \\
\hline 204 & WORKPART & Votre partenaire travaille-t-il? & $\begin{array}{l}1 \\
2 \\
3 \\
99 \\
\end{array}$ & $\begin{array}{l}\text { Oui } \\
\text { Non } \\
\text { Pas de partenaire } \\
\text { Pas de réponse } \\
\end{array}$ & $\begin{array}{l}\text { passer à } 206 \\
\text { passer à } 206 \\
\text { passer à } 206 \\
\end{array}$ \\
\hline 204 & JOBTYPEP & Type d'emploi du partenaire & \multicolumn{3}{|c|}{ Enregistrer la réponse mot à mot } \\
\hline 205 & MOINC2 & Revenu mensuel du partenaire & \multicolumn{3}{|c|}{ code 4 chiffres } \\
\hline 206 & SUPPORT & Qui soutient financièrement la famille? & $\begin{array}{l}1 \\
2 \\
3 \\
4\end{array}$ & $\begin{array}{l}\text { Personne interrogée } \\
\text { Epoux/partenaire } \\
\text { Enfants } \\
\text { Autre }\end{array}$ & \\
\hline 207 & INCTOTAL & $\begin{array}{l}\text { Total revenu familial - TOUTES } \\
\text { SOURCES }\end{array}$ & \multicolumn{3}{|c|}{ code 4 chiffres } \\
\hline
\end{tabular}

\section{Type de visite}

\begin{tabular}{|c|c|c|c|c|c|}
\hline 300 & VISIT1 & Première visite à l'AGENCE ? & $\begin{array}{l}1 \\
2 \\
\end{array}$ & $\begin{array}{l}\text { Oui } \\
\text { Non } \\
\end{array}$ & passer à 302 \\
\hline 301 & REVISIT & $\begin{array}{l}\text { Votre visite est-elle un « suivi » prévu } \\
\text { lors d'une consultation précédente? }\end{array}$ & $\begin{array}{l}1 \\
2 \\
\end{array}$ & $\begin{array}{l}\text { Oui } \\
\text { Non }\end{array}$ & \\
\hline 302 & SERVICE & Principal service & $\begin{array}{l}1 \\
2 \\
3 \\
4 \\
5 \\
6 \\
88\end{array}$ & $\begin{array}{l}\text { Gynécologie } \\
\text { Contraceptif injectable } \\
\text { Pose stérilet } \\
\text { Examen stérilet } \\
\text { Pilule } \\
\text { Soins prénatals } \\
\text { Autre }\end{array}$ & $\begin{array}{l}\text { passer à } 401 \\
\text { passer à } 501 \\
\text { passer à } 601 \\
\text { passer à } 701 \\
\text { passer à } 801 \\
\text { passer à } 901 \\
\text { passer à FIN }\end{array}$ \\
\hline
\end{tabular}




\section{Questions relatives à l'acceptation de paiement : Utilisateurs actuels}

1. Gynécologie

\begin{tabular}{|c|c|c|c|c|c|}
\hline 401 & GYNPAY & $\begin{array}{l}\text { Avez-vous payé le prix normal pour la } \\
\text { consultation? }\end{array}$ & & $\begin{array}{l}\text { Oui } \\
\text { Non } \\
\text { Pas de réponse }\end{array}$ & passer à 405 \\
\hline 402 & GYNPAID & Montant payé & \multicolumn{3}{|c|}{ code 2 chiffres } \\
\hline 403 & GYNREAS & $\begin{array}{l}\text { Raison pour laquelle le prix normal n'a } \\
\text { pas été payé }\end{array}$ & \multicolumn{3}{|c|}{ Enregistrer la réponse mot à mot } \\
\hline 405 & GYNWTPM & $\begin{array}{l}\text { Prête à payer } \mathrm{I} / 5 \text { de plus pour des } \\
\text { services de gynécologie }\end{array}$ & $\begin{array}{l}1 \\
2 \\
99\end{array}$ & $\begin{array}{l}\text { Oui } \\
\text { Non } \\
\text { Ne sait pas }\end{array}$ & $\begin{array}{l}\text { passer à } 407 \\
\text { passer à } 407\end{array}$ \\
\hline 406 & GYNWTPH & $\begin{array}{l}\text { Prête à payer } \mathrm{I} / 10 \text { de plus pour des } \\
\text { services de gynécologie }\end{array}$ & $\begin{array}{l}1 \\
2 \\
99\end{array}$ & $\begin{array}{l}\text { Oui } \\
\text { Non } \\
\text { Ne sait pas }\end{array}$ & $\begin{array}{l}\text { passer à } 408 \\
\text { passer à } 408 \\
\text { passer à } 408\end{array}$ \\
\hline 407 & GYNWTPL & $\begin{array}{l}\text { Prête à payer } \mathrm{I} / 2 \text { de plus pour des } \\
\text { services de gynécologie }\end{array}$ & $\begin{array}{l}1 \\
2 \\
99\end{array}$ & $\begin{array}{l}\text { Oui } \\
\text { Non } \\
\text { Ne sait pas }\end{array}$ & \\
\hline 408 & GYNWTPS & $\begin{array}{l}\text { Montant maximum prête à payer pour } \\
\text { des services de gynécologie }\end{array}$ & \multicolumn{3}{|c|}{ code 3 chiffres } \\
\hline 409 & GYNALT & $\begin{array}{l}\text { Que feriez-vous si le prix était trop } \\
\text { élevé? }\end{array}$ & $\begin{array}{l}1 \\
2 \\
88 \\
99 \\
\end{array}$ & $\begin{array}{l}\text { S'en passer } \\
\text { Aller ailleurs } \\
\text { Autre } \\
\text { Ne sait pas }\end{array}$ & $\begin{array}{l}\text { passer à } 1000 \\
\text { passer à } 410 \\
\text { passer à } 1000 \\
\text { passer à } 1000\end{array}$ \\
\hline 410 & GYNALTP & $\begin{array}{l}\text { Autre prestataire de services de } \\
\text { gynécologie si le prix est trop élevé }\end{array}$ & $\begin{array}{l}1 \\
2 \\
3 \\
4 \\
88 \\
99\end{array}$ & $\begin{array}{l}\text { Secteur public } \\
\text { Autre ONG } \\
\text { Secteur privé } \\
\text { Pharmacie } \\
\text { Autre } \\
\text { Ne sait pas }\end{array}$ & $\begin{array}{l}\text { passer à } 1000 \\
\text { passer à } 1000 \\
\text { passer à } 1000 \\
\text { passer à } 1000 \\
\text { passer à } 1000 \\
\text { passer à } 1000\end{array}$ \\
\hline
\end{tabular}

2. Contraceptif injectable

\begin{tabular}{|c|c|c|c|c|c|}
\hline 501 & INCPAY & $\begin{array}{l}\text { Avez-vous payé le prix normal pour la } \\
\text { consultation? }\end{array}$ & $\begin{array}{l}1 \\
2 \\
99\end{array}$ & $\begin{array}{l}\text { Oui } \\
\text { Non } \\
\text { Pas de réponse }\end{array}$ & passer à 505 \\
\hline 502 & INCPAID & Montant payé & \multicolumn{3}{|c|}{ code 3 chiffres } \\
\hline 503 & INCREAS & $\begin{array}{l}\text { Raison pour laquelle le prix normal n’a } \\
\text { pas été payé }\end{array}$ & \multicolumn{3}{|c|}{ Enregistrer la réponse mot à mot } \\
\hline 505 & INCWTPM & $\begin{array}{l}\text { Prête à payer } \mathrm{I} / 5 \text { de plus pour le } \\
\text { contraceptif injectable }\end{array}$ & $\begin{array}{l}1 \\
2 \\
99 \\
\end{array}$ & $\begin{array}{l}\text { Oui } \\
\text { Non } \\
\text { Ne sait pas }\end{array}$ & $\begin{array}{l}\text { passer à } 507 \\
\text { passer à } 507\end{array}$ \\
\hline 506 & INCWTPH & $\begin{array}{l}\text { Prête à payer I/10 de plus pour le } \\
\text { contraceptif injectable }\end{array}$ & $\begin{array}{l}1 \\
2 \\
99\end{array}$ & $\begin{array}{l}\text { Oui } \\
\text { Non } \\
\text { Ne sait pas }\end{array}$ & $\begin{array}{l}\text { passer à } 508 \\
\text { passer à } 508 \\
\text { passer à } 508\end{array}$ \\
\hline 507 & INCWTPL & $\begin{array}{l}\text { Prête à payer I/3 de plus pour le } \\
\text { contraceptif injectable }\end{array}$ & $\begin{array}{l}1 \\
2 \\
99\end{array}$ & $\begin{array}{l}\text { Oui } \\
\text { Non } \\
\text { Ne sait pas }\end{array}$ & \\
\hline 508 & INCWTPS & $\begin{array}{l}\text { Montant maximum prête à payer pour le } \\
\text { contraceptif injectable }\end{array}$ & \multicolumn{3}{|c|}{ code 3 chiffres } \\
\hline 509 & INCALT & $\begin{array}{l}\text { Que feriez-vous si le prix était trop } \\
\text { élevé ? }\end{array}$ & $\begin{array}{l}1 \\
2 \\
3 \\
88 \\
99\end{array}$ & $\begin{array}{l}\text { Pas de contraception } \\
\text { Aller ailleurs } \\
\text { Passer à un moyen moins cher } \\
\text { Autre } \\
\text { Ne sait pas }\end{array}$ & $\begin{array}{l}\text { passer à } 1000 \\
\text { passer à } 510 \\
\text { passer à } 1000 \\
\text { passer à } 1000 \\
\text { passer à } 1000 \\
\end{array}$ \\
\hline 510 & INCALTP & $\begin{array}{l}\text { Autre fournisseur de contraceptif } \\
\text { injectable si le prix est trop élevé }\end{array}$ & $\begin{array}{l}1 \\
2 \\
3 \\
4 \\
88\end{array}$ & $\begin{array}{l}\text { Secteur public } \\
\text { Autre ONG } \\
\text { Secteur privé } \\
\text { Pharmacie } \\
\text { Autre }\end{array}$ & $\begin{array}{l}\text { passer à } 1000 \\
\text { passer à } 1000 \\
\text { passer à } 1000 \\
\text { passer à } 1000 \\
\text { passer à } 1000\end{array}$ \\
\hline
\end{tabular}




\begin{tabular}{|l|l|l|l|}
\hline & & Ne sait pas & 99 \\
\hline
\end{tabular}

3. Pose de stérilet

\begin{tabular}{|c|c|c|c|c|c|}
\hline 601 & IUDPAY & $\begin{array}{l}\text { Avez-vous payé le prix normal pour la } \\
\text { consultation? }\end{array}$ & $\begin{array}{l}1 \\
2 \\
99 \\
\end{array}$ & $\begin{array}{l}\text { Oui } \\
\text { Non } \\
\text { Pas de réponse }\end{array}$ & passer à 605 \\
\hline 602 & IUDPAID & Montant payé & \multicolumn{3}{|c|}{ code 2 chiffres } \\
\hline 603 & IUDREAS & $\begin{array}{l}\text { Raison pour laquelle le prix normal n'a } \\
\text { pas été payé }\end{array}$ & \multicolumn{3}{|c|}{ Enregistrer la réponse mot à mot } \\
\hline 605 & IUDWTPM & $\begin{array}{l}\text { Prête à payer } \mathrm{I} / 5 \text { de plus pour pose de } \\
\text { stérilet }\end{array}$ & $\begin{array}{l}1 \\
2 \\
99 \\
\end{array}$ & $\begin{array}{l}\text { Oui } \\
\text { Non } \\
\text { Ne sait pas }\end{array}$ & $\begin{array}{l}\text { passer à } 607 \\
\text { passer à } 607\end{array}$ \\
\hline 606 & IUDWTPH & $\begin{array}{l}\text { Prête à payer I/10 de plus pour pose de } \\
\text { stérilet }\end{array}$ & $\begin{array}{l}1 \\
2 \\
99 \\
\end{array}$ & $\begin{array}{l}\text { Oui } \\
\text { Non } \\
\text { Ne sait pas }\end{array}$ & $\begin{array}{l}\text { passer à } 608 \\
\text { passer à } 608 \\
\text { passer à } 608\end{array}$ \\
\hline 607 & IUDWTPL & $\begin{array}{l}\text { Prête à payer } \mathrm{I} / 3 \text { de plus pour pose de } \\
\text { stérilet }\end{array}$ & & $\begin{array}{l}\text { Oui } \\
\text { Non } \\
\text { Ne sait pas }\end{array}$ & \\
\hline 608 & IUDWTPS & $\begin{array}{l}\text { Montant maximum prête à payer pour } \\
\text { pose de stérilet }\end{array}$ & \multicolumn{3}{|c|}{ code 3 chiffres } \\
\hline 609 & IUDALT & $\begin{array}{l}\text { Que feriez-vous si le prix était trop } \\
\text { élevé ? }\end{array}$ & $\begin{array}{l}1 \\
2 \\
3 \\
88 \\
99 \\
\end{array}$ & $\begin{array}{l}\text { Pas de contraception } \\
\text { Aller ailleurs } \\
\text { Passer à un moyen moins cher } \\
\text { Autre } \\
\text { Ne sait pas }\end{array}$ & $\begin{array}{l}\text { passer à } 1000 \\
\text { passer à } 610 \\
\text { r passer à } 1000 \\
\text { passer à } 1000 \\
\text { passer à } 1000\end{array}$ \\
\hline 610 & INCALTP & $\begin{array}{l}\text { Autre prestataire pour pose de stérilet si } \\
\text { le prix est trop élevé }\end{array}$ & $\begin{array}{l}1 \\
2 \\
3 \\
4 \\
88 \\
99\end{array}$ & $\begin{array}{l}\text { Secteur public } \\
\text { Autre ONG } \\
\text { Secteur privé } \\
\text { Pharmacie } \\
\text { Autre } \\
\text { Ne sait pas }\end{array}$ & $\begin{array}{l}\text { passer à } 1000 \\
\text { passer à } 1000 \\
\text { passer à } 1000 \\
\text { passer à } 1000 \\
\text { passer à } 1000 \\
\text { passer à } 1000\end{array}$ \\
\hline
\end{tabular}

\section{Examen stérilet}

\begin{tabular}{|c|c|c|c|c|c|}
\hline 701 & IREPAY & $\begin{array}{l}\text { Avez-vous payé le montant normal pour } \\
\text { la consultation? }\end{array}$ & $\begin{array}{l}1 \\
2 \\
99\end{array}$ & $\begin{array}{l}\text { Oui } \\
\text { Non } \\
\text { Ne sait pas }\end{array}$ & passer à 705 \\
\hline 702 & IREPAID & Montant payé & \multicolumn{3}{|c|}{ code 2 chiffres } \\
\hline 703 & IREREAS & $\begin{array}{l}\text { Raison pour laquelle le prix normal n'a } \\
\text { pas été payé }\end{array}$ & \multicolumn{3}{|c|}{ Enregistrer la réponse mot à mot } \\
\hline 705 & IREWTPM & $\begin{array}{l}\text { Prête à payer } \mathrm{I} / 5 \text { de plus pour un examen } \\
\text { stérilet }\end{array}$ & $\begin{array}{l}1 \\
2 \\
99 \\
\end{array}$ & $\begin{array}{l}\text { Oui } \\
\text { Non } \\
\text { Ne sait pas }\end{array}$ & $\begin{array}{l}\text { passer à } 707 \\
\text { passer à } 707\end{array}$ \\
\hline 706 & IREWTPH & $\begin{array}{l}\text { Prête à payer } \mathrm{I} / 10 \text { de plus pour un } \\
\text { examen stérilet }\end{array}$ & $\begin{array}{l}1 \\
2 \\
99 \\
\end{array}$ & $\begin{array}{l}\text { Oui } \\
\text { Non } \\
\text { Ne sait pas } \\
\end{array}$ & $\begin{array}{l}\text { passer à } 708 \\
\text { passer à } 708 \\
\text { passer à } 708\end{array}$ \\
\hline 707 & IREWTPL & $\begin{array}{l}\text { Prête à payer } \mathrm{I} / 2 \text { de plus pour un examen } \\
\text { stérilet }\end{array}$ & $\begin{array}{l}1 \\
2 \\
99 \\
\end{array}$ & $\begin{array}{l}\text { Oui } \\
\text { Non } \\
\text { Ne sait pas }\end{array}$ & \\
\hline 708 & IREWTPS & $\begin{array}{l}\text { Montant maximum prête à payer pour un } \\
\text { examen stérilet }\end{array}$ & \multicolumn{3}{|c|}{ code 3 chiffres } \\
\hline 709 & IREALT & $\begin{array}{l}\text { Que feriez-vous si le prix était trop } \\
\text { élevé ? }\end{array}$ & $\begin{array}{l}1 \\
2 \\
88 \\
99\end{array}$ & $\begin{array}{l}\text { S'en passer } \\
\text { Aller ailleurs } \\
\text { Autre } \\
\text { Ne sait pas }\end{array}$ & $\begin{array}{l}\text { passer à } 1000 \\
\text { passer à } 710 \\
\text { passer à } 1000 \\
\text { passer à } 1000\end{array}$ \\
\hline 710 & IREALTP & $\begin{array}{l}\text { Autre prestataire d'examen stérilet si le } \\
\text { prix était trop élevé }\end{array}$ & $\begin{array}{l}1 \\
2 \\
3 \\
4 \\
88\end{array}$ & $\begin{array}{l}\text { Secteur public } \\
\text { Autre ONG } \\
\text { Secteur privé } \\
\text { Pharmacie } \\
\text { Autre }\end{array}$ & $\begin{array}{l}\text { passer à } 1000 \\
\text { passer à } 1000 \\
\text { passer à } 1000 \\
\text { passer à } 1000 \\
\text { passer à } 1000\end{array}$ \\
\hline
\end{tabular}




\begin{tabular}{|l|l|l|ll}
\hline & & & Ne sait pas & passer à 1000 \\
\hline
\end{tabular}

5. Achat de la pilule

\begin{tabular}{|c|c|c|c|c|c|}
\hline 801 & OCPAY & $\begin{array}{l}\text { Avez-vous payé le prix normal pour la } \\
\text { consultation? }\end{array}$ & & $\begin{array}{l}\text { Oui } \\
\text { Non } \\
\text { Ne sait pas }\end{array}$ & passer à 804 \\
\hline 802 & OCPAID & Montant payé & \multicolumn{3}{|c|}{ code 2 chiffres } \\
\hline 803 & OCREAS & $\begin{array}{l}\text { Raison pour laquelle le prix normal n'a } \\
\text { pas été payé }\end{array}$ & \multicolumn{3}{|c|}{ Enregistrer la réponse mot à mot } \\
\hline 805 & OCWTPM & $\begin{array}{l}\text { Prête à payer } \mathrm{I} / 2 \text { de plus pour des } \\
\text { contraceptifs par voie orale }\end{array}$ & $\begin{array}{l}1 \\
2 \\
99\end{array}$ & $\begin{array}{l}\text { Oui } \\
\text { Non } \\
\text { Ne sait pas }\end{array}$ & $\begin{array}{l}\text { passer à } 807 \\
\text { passer à } 807\end{array}$ \\
\hline 806 & OCWTPH & $\begin{array}{l}\text { Prête à payer } \mathrm{I} / 3 \text { de plus pour des } \\
\text { contraceptifs par voie orale }\end{array}$ & $\begin{array}{l}1 \\
2 \\
99\end{array}$ & $\begin{array}{l}\text { Oui } \\
\text { Non } \\
\text { Ne sait pas }\end{array}$ & $\begin{array}{l}\text { passer à } 808 \\
\text { passer à } 808 \\
\text { passer à } 808\end{array}$ \\
\hline 807 & OCWTPL & $\begin{array}{l}\text { Prête à payer I/1 de plus pour des } \\
\text { contraceptifs par voie orale }\end{array}$ & & $\begin{array}{l}\text { Oui } \\
\text { Non } \\
\text { Ne sait pas }\end{array}$ & \\
\hline 808 & OCWTPS & $\begin{array}{l}\text { Montant maximum prête à payer pour } \\
\text { des contraceptifs par voie orale }\end{array}$ & \multicolumn{3}{|c|}{ code 3 chiffres } \\
\hline 809 & OCALT & $\begin{array}{l}\text { Que feriez-vous si le prix était trop } \\
\text { élevé ? }\end{array}$ & $\begin{array}{l}1 \\
2 \\
3 \\
88 \\
99 \\
\end{array}$ & $\begin{array}{l}\text { Pas de contraception } \\
\text { Aller ailleurs } \\
\text { Passer à un moyen mo } \\
\text { Autre } \\
\text { Ne sait pas }\end{array}$ & $\begin{array}{l}\text { passer à } 1000 \\
\text { passer à } 810 \\
\text { r passer à } 1000 \\
\text { passer à } 1000 \\
\text { passer à } 1000\end{array}$ \\
\hline 810 & OCALTP & $\begin{array}{l}\text { Autre fournisseur de contraceptifs par } \\
\text { voie si le prix était trop élevé }\end{array}$ & $\begin{array}{l}1 \\
2 \\
3 \\
4 \\
88 \\
99 \\
\end{array}$ & $\begin{array}{l}\text { Secteur public } \\
\text { Autre ONG } \\
\text { Secteur privé } \\
\text { Pharmacie } \\
\text { Autre } \\
\text { Ne sait pas }\end{array}$ & $\begin{array}{l}\text { passer à } 1000 \\
\text { passer à } 1000 \\
\text { passer à } 1000 \\
\text { passer à } 1000 \\
\text { passer à } 1000 \\
\text { passer à } 1000 \\
\end{array}$ \\
\hline
\end{tabular}

6. Soins prénatals

\begin{tabular}{|c|c|c|c|c|c|}
\hline 901 & PNCPAY & $\begin{array}{l}\text { Avez-vous payé le prix normal pour la } \\
\text { consultation? }\end{array}$ & $\begin{array}{l}1 \\
2 \\
99\end{array}$ & $\begin{array}{l}\text { Oui } \\
\text { Non } \\
\text { Ne sait pas }\end{array}$ & passer à 905 \\
\hline 902 & PNCPAID & Montant payé & \multicolumn{3}{|c|}{ code 3 chiffres } \\
\hline 903 & PNCREAS & $\begin{array}{l}\text { Raison pour laquelle le prix normal n’a } \\
\text { pas été payé }\end{array}$ & \multicolumn{3}{|c|}{ Enregistrer la réponse mot à mot } \\
\hline 905 & PNCWTPM & $\begin{array}{l}\text { Prête à payer } \mathrm{I} / 5 \text { de plus pour des soins } \\
\text { prénatals }\end{array}$ & $\begin{array}{l}1 \\
2 \\
99 \\
\end{array}$ & $\begin{array}{l}\text { Oui } \\
\text { Non } \\
\text { Ne sait pas }\end{array}$ & $\begin{array}{l}\text { passer à } 907 \\
\text { passer à } 907\end{array}$ \\
\hline 906 & PNCWTPH & $\begin{array}{l}\text { Prête à payer } \mathrm{I} / 10 \text { de plus pour des soins } \\
\text { prénatals }\end{array}$ & $\begin{array}{l}1 \\
2 \\
99 \\
\end{array}$ & $\begin{array}{l}\text { Oui } \\
\text { Non } \\
\text { Ne sait pas }\end{array}$ & $\begin{array}{l}\text { passer à } 908 \\
\text { passer à } 908 \\
\text { passer à } 908\end{array}$ \\
\hline 907 & PNCWTPL & $\begin{array}{l}\text { Prête à payer } \mathrm{I} / 2 \text { de plus pour des oins } \\
\text { prénatals }\end{array}$ & & $\begin{array}{l}\text { Oui } \\
\text { Non } \\
\text { Ne sait pas }\end{array}$ & \\
\hline 908 & PNCWTPS & $\begin{array}{l}\text { Montant maximum prête à payer pour } \\
\text { des soins prénatals }\end{array}$ & \multicolumn{3}{|c|}{ code 3 chiffres } \\
\hline 909 & PNCALT & $\begin{array}{l}\text { Que feriez-vous si le prix était trop } \\
\text { élevé? }\end{array}$ & $\begin{array}{l}1 \\
2 \\
88 \\
99\end{array}$ & $\begin{array}{l}\text { S'en passer } \\
\text { Aller ailleurs } \\
\text { Autre } \\
\text { Ne sait pas }\end{array}$ & $\begin{array}{l}\text { passer à } 1000 \\
\text { passer à } 910 \\
\text { passer à } 1000 \\
\text { passer à } 1000\end{array}$ \\
\hline 910 & PNCALTP & $\begin{array}{l}\text { Autre prestataire de soins prénatals si le } \\
\text { prix était trop élevé }\end{array}$ & $\begin{array}{l}1 \\
2 \\
3 \\
4\end{array}$ & $\begin{array}{l}\text { Secteur public } \\
\text { Autre ONG } \\
\text { Secteur privé } \\
\text { Pharmacie }\end{array}$ & $\begin{array}{l}\text { passer à } 1000 \\
\text { passer à } 1000 \\
\text { passer à } 1000 \\
\text { passer à } 1000\end{array}$ \\
\hline
\end{tabular}




\begin{tabular}{|l|l|l|ll|}
\hline & & passer à 1000 & 88 & Autre \\
& & & Ne sait pas & passer à $1000 \quad$ \\
\hline
\end{tabular}

\section{E. Questions relatives à l'acceptation de paiement : Utilisateurs potentiels}

1. Stérilisation féminine

\begin{tabular}{|c|c|c|c|c|c|}
\hline 1000 & VSCKNOW & $\begin{array}{l}\text { Entendu parler de la contraception } \\
\text { chirurgicale volontaire? }\end{array}$ & $\begin{array}{l}1 \\
2 \\
99 \\
\end{array}$ & $\begin{array}{l}\text { Oui } \\
\text { Non } \\
\text { Ne sait pas }\end{array}$ & $\begin{array}{l}\text { passer à } 1100 \\
\text { passer à } 1100\end{array}$ \\
\hline 1001 & VSCINT & $\begin{array}{l}\text { Intérêt futur à la CCV (dans les } 2 \\
\text { prochaines années) }\end{array}$ & $\begin{array}{l}1 \\
2 \\
3 \\
99\end{array}$ & $\begin{array}{l}\text { Oui } \\
\text { Non } \\
\text { Déjà fait } \\
\text { Ne sait pas }\end{array}$ & $\begin{array}{l}\text { passer à } 1100 \\
\text { passer à } 1100 \\
\text { passer à } 1100\end{array}$ \\
\hline 1003 & VSCWTPM & Prête à payer I/200 pour l'opération & $\begin{array}{l}1 \\
2 \\
99\end{array}$ & $\begin{array}{l}\text { Oui } \\
\text { Non } \\
\text { Ne sait pas }\end{array}$ & $\begin{array}{l}\text { passer à } 1105 \\
\text { passer à } 1105\end{array}$ \\
\hline 1004 & VSCWTPH & Prête à payer I/300 pour l'opération & $\begin{array}{l}1 \\
2 \\
99\end{array}$ & $\begin{array}{l}\text { Oui } \\
\text { Non } \\
\text { Ne sait pas }\end{array}$ & $\begin{array}{l}\text { passer à } 1106 \\
\text { passer à } 1106 \\
\text { passer à } 1106\end{array}$ \\
\hline 1005 & VSCWTPL & Prête à payer I/100 pour l'opération & $\begin{array}{l}1 \\
2 \\
99\end{array}$ & $\begin{array}{l}\text { Oui } \\
\text { Non } \\
\text { Ne sait pas }\end{array}$ & \\
\hline 1006 & VSCWTPS & Maximum prête à payer pour l'opération & \multicolumn{3}{|c|}{ code 4 chiffres } \\
\hline 1007 & VSCALT & Autre que CCV si le prix est trop élevé & $\begin{array}{l}1 \\
2 \\
88 \\
99 \\
\end{array}$ & $\begin{array}{l}\text { S'en passer } \\
\text { Aller ailleurs } \\
\text { Autre } \\
\text { Ne sait pas }\end{array}$ & $\begin{array}{l}\text { passer à } 1008 \\
\text { passer à } 1009 \\
\text { passer à } 1100 \\
\text { passer à } 1100\end{array}$ \\
\hline 1008 & CONTCONT & $\begin{array}{l}\text { Si pas d'opération, poursuite de } \\
\text { l'utilisation de la contraception? }\end{array}$ & $\begin{array}{l}1 \\
2 \\
99\end{array}$ & $\begin{array}{l}\text { Oui } \\
\text { Non } \\
\text { Ne sait pas }\end{array}$ & $\begin{array}{l}\text { passer à } 1100 \\
\text { passer à } 1100 \\
\text { passer à } 1100\end{array}$ \\
\hline 1009 & VSCALTP & $\begin{array}{l}\text { Autre prestataire CCV si le prix était trop } \\
\text { élevé }\end{array}$ & $\begin{array}{l}1 \\
2 \\
3 \\
88 \\
99\end{array}$ & $\begin{array}{l}\text { Secteur public } \\
\text { Autre ONG } \\
\text { Secteur privé } \\
\text { Autre } \\
\text { Ne sait pas }\end{array}$ & \\
\hline
\end{tabular}

\section{F. Acceptation de paiement pour l'amélioration des accommodements et de la qualité}

1. Acceptation de paiement pour réduction du temps d'attente

\begin{tabular}{|c|c|c|c|c|c|}
\hline 1100 & WAITTIME & Temps passé à attendre avant de voir & \multicolumn{3}{|c|}{ Temps en minutes } \\
\hline 1101 & WTPERC & $\begin{array}{l}\text { Quel est votre avis sur le temps } \\
\text { d'attente? }\end{array}$ & $\begin{array}{l}1 \\
2 \\
3\end{array}$ & $\begin{array}{l}\text { Excessif } \\
\text { Raisonnable } \\
\text { Court }\end{array}$ & \\
\hline 1102 & WTWTPM & $\begin{array}{l}\text { Prêt à payer } \mathrm{I} / 3 \text { de plus pour attendre } \\
\text { moins longtemps }\end{array}$ & $\begin{array}{l}1 \\
2 \\
99 \\
\end{array}$ & $\begin{array}{l}\text { Oui } \\
\text { Non } \\
\text { Ne sait pas }\end{array}$ & $\begin{array}{l}\text { passer à } 1104 \\
\text { passer à } 1104\end{array}$ \\
\hline 1103 & WTWTPH & $\begin{array}{l}\text { Prêt à payer } \mathrm{I} / 6 \text { de plus pour attendre } \\
\text { moins longtemps }\end{array}$ & $\begin{array}{l}1 \\
2 \\
99\end{array}$ & $\begin{array}{l}\text { Oui } \\
\text { Non } \\
\text { Ne sait pas }\end{array}$ & $\begin{array}{l}\text { passer à } 1105 \\
\text { passer à } 1105 \\
\text { passer à } 1105\end{array}$ \\
\hline 1104 & WTWTPL & $\begin{array}{l}\text { Prêt à payer } \mathrm{I} / 1 \mathrm{de} \text { plus pour attendre } \\
\text { moins longtemps }\end{array}$ & & $\begin{array}{l}\text { Oui } \\
\text { Non } \\
\text { Ne sait pas }\end{array}$ & \\
\hline 1105 & WTWTPS & $\begin{array}{l}\text { Montant maximum prêt à payer pour } \\
\text { attendre moins longtemps }\end{array}$ & & chiffres & \\
\hline
\end{tabular}


2. Acceptation de paiement pour le counseling

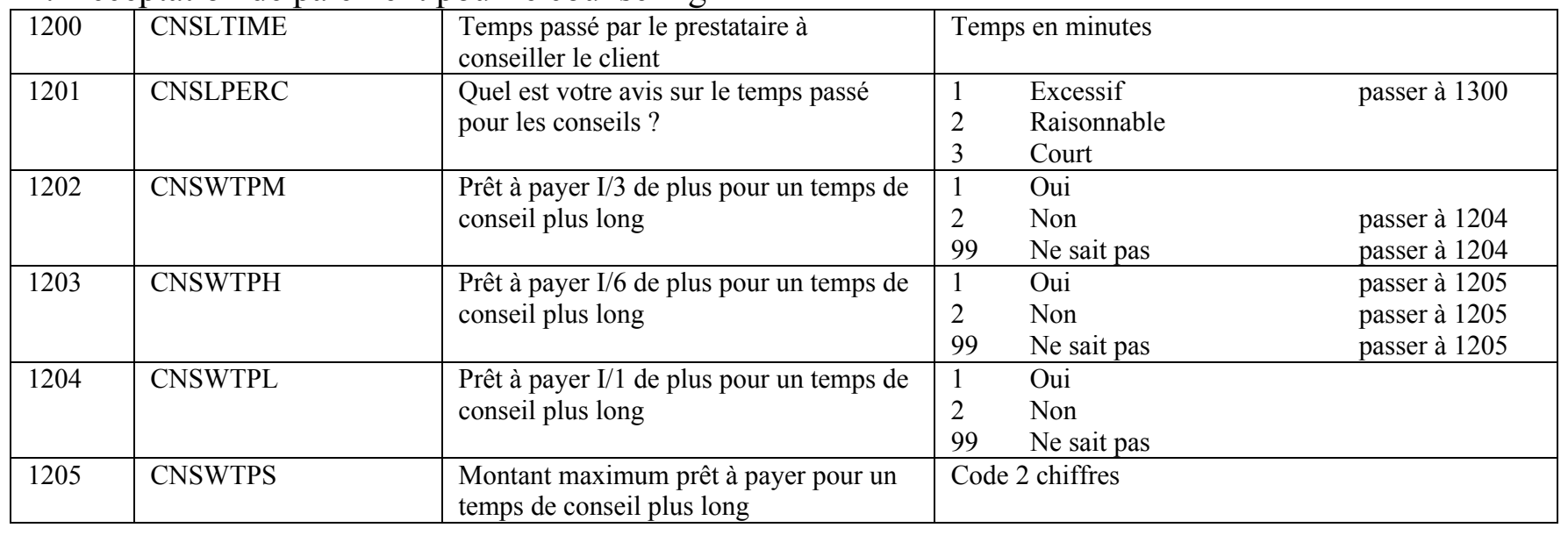

3. Acceptation de paiement pour amélioration du dispensaire

\begin{tabular}{|c|c|c|c|c|c|}
\hline 1301 & WRCHAIR & $\begin{array}{l}\text { Importance pour la personne interrogée } \\
\text { d'avoir des chaises plus confortables } \\
\text { dans la salle d'attente }\end{array}$ & $\begin{array}{l}1 \\
2 \\
3 \\
4 \\
5\end{array}$ & Pas très important & \\
\hline 1301 & WRAC & $\begin{array}{l}\text { Importance pour la personne interrogée } \\
\text { d'avoir une salle d'attente climatisée }\end{array}$ & $\begin{array}{l}1 \\
2 \\
3 \\
4 \\
5\end{array}$ & $\begin{array}{l}\text { Pas très important } \\
\text { Très important }\end{array}$ & \\
\hline 1301 & WRTV & $\begin{array}{l}\text { Importance pour la personne interrogée } \\
\text { d'avoir la télévision dans la salle } \\
\text { d'attente }\end{array}$ & $\begin{array}{l}1 \\
2 \\
3 \\
4 \\
5\end{array}$ & $\begin{array}{l}\text { Pas très important } \\
\text { Très important }\end{array}$ & \\
\hline 1302 & MOSTIMP & $\begin{array}{l}\text { Amélioration la plus importante pour la } \\
\text { personne interrogée }\end{array}$ & $\begin{array}{l}1 \\
2 \\
3\end{array}$ & $\begin{array}{l}\text { Chaises plus confortables } \\
\text { Climatisation } \\
\text { Télévision }\end{array}$ & $\begin{array}{l}\text { passer à } 1303 \\
\text { passer à } 1307 \\
\text { passer à } 1311\end{array}$ \\
\hline 1303 & CHWTPM & $\begin{array}{l}\text { Prêt à payer } \mathrm{I} / 3 \text { de plus pour avoir des } \\
\text { chaises plus confortables dans la salle } \\
\text { d'attente }\end{array}$ & $\begin{array}{l}1 \\
2 \\
99 \\
\end{array}$ & $\begin{array}{l}\text { Oui } \\
\text { Non } \\
\text { Ne sait pas }\end{array}$ & $\begin{array}{l}\text { passer à } 1305 \\
\text { passer à } 1305\end{array}$ \\
\hline 1304 & CHWTPH & $\begin{array}{l}\text { Prêt à payer I/ } 6 \text { de plus pour avoir des } \\
\text { chaises plus confortables dans la salle } \\
\text { d'attente }\end{array}$ & $\begin{array}{l}1 \\
2 \\
99\end{array}$ & $\begin{array}{l}\text { Oui } \\
\text { Non } \\
\text { Ne sait pas }\end{array}$ & $\begin{array}{l}\text { passer à } 1306 \\
\text { passer à } 1306 \\
\text { passer à } 1306\end{array}$ \\
\hline 1305 & CHWTPL & $\begin{array}{l}\text { Prêt à payer I/ } 1 \text { de plus pour avoir des } \\
\text { chaises plus confortables dans la salle } \\
\text { d'attente }\end{array}$ & $\begin{array}{l}1 \\
2 \\
99\end{array}$ & $\begin{array}{l}\text { Oui } \\
\text { Non } \\
\text { Ne sait pas }\end{array}$ & \\
\hline 1306 & CHWTPS & $\begin{array}{l}\text { Montant maximum prêt à payer pour } \\
\text { avoir des chaises plus confortables dans } \\
\text { la salle d'attente }\end{array}$ & \multicolumn{3}{|c|}{ code 2 chiffres } \\
\hline 1307 & ACWTPM & $\begin{array}{l}\text { Prêt à payer } \mathrm{I} / 3 \text { de plus pour avoir la } \\
\text { climatisation dans la salle d'attente }\end{array}$ & $\begin{array}{l}1 \\
2 \\
99 \\
\end{array}$ & $\begin{array}{l}\text { Oui } \\
\text { Non } \\
\text { Ne sait pas }\end{array}$ & $\begin{array}{l}\text { passer à } 1309 \\
\text { passer à } 1309\end{array}$ \\
\hline 1308 & ACWTPH & $\begin{array}{l}\text { Prêt à payer } \mathrm{I} / 6 \text { de plus pour avoir la } \\
\text { climatisation dans la salle d'attente }\end{array}$ & $\begin{array}{l}1 \\
2 \\
99 \\
\end{array}$ & $\begin{array}{l}\text { Oui } \\
\text { Non } \\
\text { Ne sait pas }\end{array}$ & $\begin{array}{l}\text { passer à } 1310 \\
\text { passer à } 1310 \\
\text { passer à } 1310\end{array}$ \\
\hline 1309 & ACWTPL & $\begin{array}{l}\text { Prêt à payer } \mathrm{I} / 1 \text { de plus pour avoir la } \\
\text { climatisation dans la salle d'attente }\end{array}$ & $\begin{array}{l}1 \\
2 \\
99 \\
\end{array}$ & $\begin{array}{l}\text { Oui } \\
\text { Non } \\
\text { Ne sait pas }\end{array}$ & \\
\hline
\end{tabular}




\begin{tabular}{|c|c|c|c|c|c|}
\hline 1310 & ACWTPS & Montant maximum prêt à payer pour & \multicolumn{3}{|c|}{ code 2 chiffres } \\
\hline 1311 & TVWTPM & $\begin{array}{l}\text { Prêt à payer } \mathrm{I} / 3 \text { de plus pour avoir la } \\
\text { télévision dans la salle d'attente }\end{array}$ & $\begin{array}{l}1 \\
2 \\
99\end{array}$ & $\begin{array}{l}\text { Oui } \\
\text { Non } \\
\text { Ne sait pas }\end{array}$ & $\begin{array}{l}\text { passer à } 1313 \\
\text { passer à } 1313\end{array}$ \\
\hline 1312 & TVWTPH & $\begin{array}{l}\text { Prêt à payer } \mathrm{I} / 6 \text { de plus pour avoir la } \\
\text { télévision dans la salle d'attente }\end{array}$ & $\begin{array}{l}1 \\
2 \\
99 \\
\end{array}$ & $\begin{array}{l}\text { Oui } \\
\text { Non } \\
\text { Ne sait pas }\end{array}$ & $\begin{array}{l}\text { passer à } 1314 \\
\text { passer à } 1314 \\
\text { passer à } 1314\end{array}$ \\
\hline 1313 & TVWTPL & $\begin{array}{l}\text { prêt à payer I/1 de plus pour avoir la } \\
\text { télévision dans la salle d'attente }\end{array}$ & & $\begin{array}{l}\text { Oui } \\
\text { Non } \\
\text { Ne sait pas }\end{array}$ & \\
\hline 1314 & TVWTPS & $\begin{array}{l}\text { Montant maximum prêt à payer pour } \\
\text { avoir la télévision dans la salle d'attente }\end{array}$ & $\operatorname{coc}$ & chiffres & \\
\hline
\end{tabular}

\begin{tabular}{|l|l|l|lc}
\hline FIN & FIN & FIN & FIN & FIN \\
\hline
\end{tabular}

